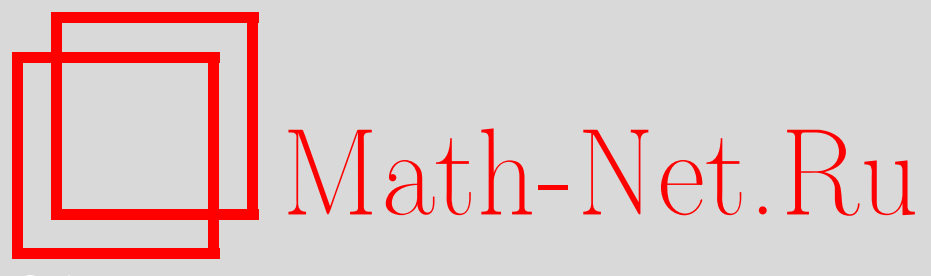

М. 3. Ровинский, Группы автоморфизмов полей и их представления, УМH, 2007, том 62, выпуск 6, 87-156

DOI: https://doi.org/10.4213/rm8515

Использование Общероссийского математического портала Math-Net.Ru подразумевает, что вы прочитали и согласны с пользовательским соглашением http://www . mathnet.ru/rus/agreement

Параметры загрузки:

IP: 54.224 .60 .19

26 апреля 2023 г., 16:45:10

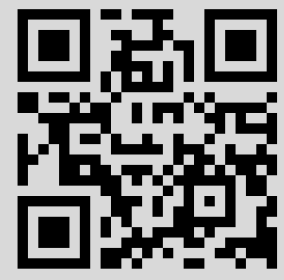




\section{Группы автоморфизмов полей и их представления}

\section{М. З. Ровинский}

Изучается группа автоморфизмов $G$ расширения $F \mid k$ алгебраически замкнутых полей, а также ее гладкие линейные и полулинейные представления. Объясняется связь теории представлений группы $G$ с бирациональной геометрией, алгебраическими циклами, мотивами и другими геометрическими вопросами.

Библиография: 44 названия.

\section{СоДЕРЖАНИЕ}

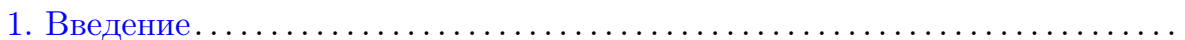

1.1. Как переводить геометрические вопросы на язык теории пред-

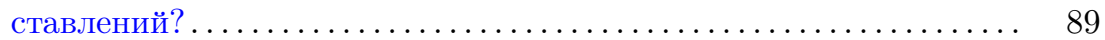

1.2. От линейных представлений к полулинейным .............. 99

1.3. Обозначения, соглашения и терминология................ 106

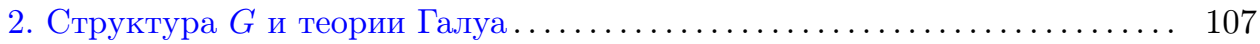

2.1. Теория Галуа для компактных подгрупп..................... 108

2.2. Топологическая простота $G^{\circ}$ и $G \ldots \ldots \ldots \ldots \ldots \ldots \ldots \ldots \ldots \ldots \ldots \ldots \ldots$

2.3. Открытые и максимальные собственные подгруппы; теории Галуа..................................... 112

2.4. Нормирования и связанные с ними подгруппы ............. 114

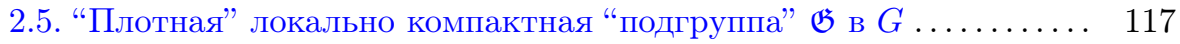

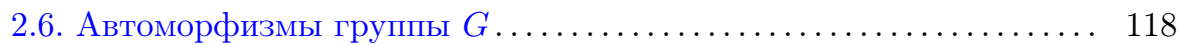

3. Общие свойства гладких представлений группы $G$ и их реализации... 118

3.1. Алгебры Гекке и соответствия .................... 123

3.2. Инварианты подгрупп и тензорные произведения ........... 126

3.3. $G$ - и $G^{\circ}$-модули вида $A(F) / A(k)$, где $A$ - коммутативная $k$-группа, морфизмы между ними и сепарабельное замыкание одномерного расширения $k \ldots \ldots \ldots \ldots \ldots \ldots \ldots \ldots \ldots \ldots \ldots \ldots \ldots \ldots \ldots \ldots \ldots \ldots \ldots \ldots$

Работа выполнена при финансовой поддержке РФФИ (гранты № 06-01-72550-НЦНИЛ_а, 07-01-92211-НЦНИЛ_а) и INTAS (грант № 05-1000008-8118). На протяжении работы над представленным здесь материалом автор получал финансовую поддержку от следующих организаций: Европейский Постдокторальный Институт, CNRS, Институт им. Макса Планка, фонд им. Александра фон Гумбольдта, фонд Бальзана (приз по математике 2004 г. Пьера Делиня).

(C) М. 3. Ровинский, 2007 
3.4. Доминантная топология, ацикличность некоторых из гладких представлений $G$ и бесконечность когомологической размерно-

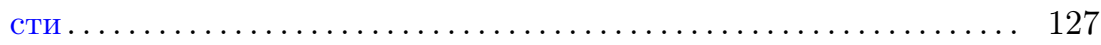

3.5. Представления, коиндуцированные с открытых подгрупп . . . . . 130

4. Гомотопически инвариантные представления группы $G \ldots \ldots \ldots \ldots \ldots$.

4.1. Категория $\mathscr{I}_{G}$, фильтрация $N$ • и дифференциальные формы . . 135

4.2. "Формула Кюннета" и тензорная структура ................. 139

4.3. Геометрическая конструкция допустимых представлений . . . . . 141

4.4. Нормирования и связанные с ними функторы ............... 146

4.5. Ограничения объектов $\mathscr{I}_{G}$ на специальные подгруппы Галуа

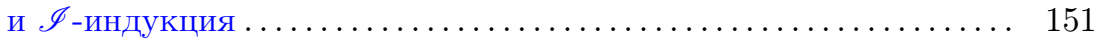

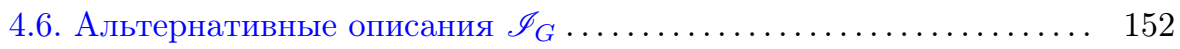

Список литературы . . . . . . . . . . . . . . . . . . . . . . . . 153

\section{1. Введение}

Изучение групп автоморфизмов полей - старый предмет. Не пытаясь описать его разветвленную историю, заметим только, что многие топологические группы являются группами автоморфизмов расширений полей. Так, автоморфизмы алгебраических расширений образуют обычные группы Галуа, а автоморфизмы полей функций алгебраических многообразий над топологическими полями содержат группы точек алгебраических групп над этими полями. Кроме того, группы точек $p$-адических групп при $p<\infty$ (а также над конечными аделями) возникают еще как группы автоморфизмов полей автоморфных функций. Изучались также группы непрерывных автоморфизмов топологических полей, например рядов Лорана.

Пусть $F \mid k$ - расширение полей счетной (что будет основным случаем) или конечной степени трансцендентности $n$, где $0 \leqslant n \leqslant \infty$, и $G=G_{F \mid k}$ - группа его автоморфизмов.

Следуя очень общей идее о том, что “достаточно симметричная" (математическая, физическая или иная) система определяется представлением своей группы симметрий, можно попытаться сравнить различные "геометрические категории над $k$ ", в которых $F$ интерпретируется как "предельный объект", с различными категориями представлений $G$.

Чтобы теория представлений группы $G$ была достаточно богатой, поле $F$ должно быть “достаточно большим". По этой причине $F$ будет обычно алгебраически замкнутым. Таким образом, $F$ - "поле функций универсальной башни $k$-многообразий размерности не выше $n$ ".

Некоторые общие обозначения, соглашения и цели. Пусть $F \mid k$ - расширение счетной или конечной степени трансцендентности $n, 1 \leqslant n \leqslant \infty$. Чтобы избежать рассмотрения уже достаточно сложной теории Галуа, поля $F$ и $k$ будут по умолчанию считаться алгебраически замкнутыми и нулевой характеристики (исключения оговариваются явно). Следуя [1]-[4] (и обобщая 
случай алгебраического расширения из [5]), мы снабжаем группу его автоморфизмов $G=G_{F \mid k}$ топологией, базу открытых подгрупп которой составляют стабилизаторы конечных подмножеств $F$. Пусть $E$ - поле характеристики 0 .

Мы исследуем структуру группы $G$, ее $E$-линейные и $F$-полулинейные представления с открытыми стабилизаторами и их связи с бирациональной геометрией, мотивами, дифференциальными формами и пучками. В частности, мы изучаем аналоги известных результатов теории представлений локально компактных (в особенности, $p$-адических) групп в случае группы $G$.

\section{1. Как переводить геометрические вопросы на язык теории пред-} ставлений? В зависимости от вида геометрических вопросов, мы будем рассматривать одну из следующих четырех категорий представлений группы $G$ : $\Phi_{G} \subset \mathscr{S} m_{G} \supset \mathscr{I}_{G} \supset \mathscr{A} d m$, приблизительно соответствующих бирациональной геометрии над $k$, ее более ограничительному ("менее функториальному") варианту, бирациональным мотивным вопросам, таким как структура групп Чжоу 0-циклов, и "конечномерным" бирациональным мотивным вопросам, таким как описание "классических" мотивных категорий.

$\mathscr{S} m_{G}$. Как правило, “алгебро-геометрические данные" $D$ над $F$ состоят из конечного числа полиномиальных уравнений, в которых участвует конечное число коэффициентов $a_{1}, \ldots, a_{N} \in F$, и группа $G$ действует на множестве "аналогичных" данных. Тогда стабилизатор $D$ в $G$ открыт, поскольку содержит $G_{F \mid k\left(a_{1}, \ldots, a_{N}\right)}$.

Пример таких данных доставляют $F$-подмногообразия какого-нибудь $k$-многообразия $X$.

В частности, $\mathbb{Q}$-векторное пространство $\mathbb{Q}[X(F)]$ 0-циклов на $X_{F}:=X \times_{k} F$ является $G$-модулем. Это представление огромно, но с него мы только начинаем.

Заметим, что оно - гладкое, т. е. его стабилизаторы открыты, так что все представления, которые мы будем рассматривать, будут гладкими.

Обратно, любое гладкое представление $G$ с циклическим вектором является фактором $G$-модуля “общих" 0-циклов на $X_{F}$, т. е. 0-циклов вне объединения дивизоров на $X$, определенных над $k$, для подходящего неприводимого многообразия $X$ размерности $\leqslant n$ над $k$. Это следует из леммы 3.1.4. Этот модуль есть

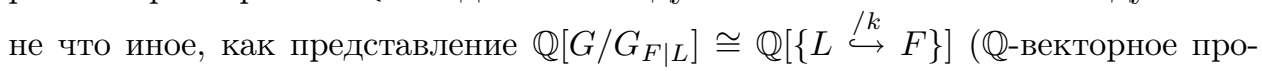
странство с базисом, заданным множеством всех вложений поля $L$ в $F$ над $k)$, индуцированное тривиальным представлением $G_{F \mid L}$ для некоторого расширения конечного типа $L \mid k$ в $F$.

ЗАмечание 1.1.1. (i) Поскольку $\mathbb{Q}[X(F)]=\bigoplus_{x \in X} \mathbb{Q}[\{k(x) \stackrel{/ k}{\hookrightarrow} F\}]$, представление $\mathbb{Q}[X(F)]$ отражает, скорее, класс многообразия $X$ в группе Гротендика $K_{0}\left(\operatorname{Var}_{k}\right)$ разбиений многообразий над $k$, чем само $X$.

(ii) Автору неизвестно, определяется ли бирациональный тип многообразия $X$ представлением $\mathbb{Q}[\{k(X) \stackrel{/ k}{\hookrightarrow} F\}]$ общих 0-циклов на $X_{F}$ группы $G$. Существуют не бирациональные многообразия $X$ и $Y, G$-модули общих 0-циклов которых имеют одни и те же неприводимые подфакторы (см. п. 3.5). Например, 
если отображение $X \rightarrow Y$ конечно в общей точке, то обратный образ индуцирует вложение $\mathbb{Q}[\{k(Y) \stackrel{/ k}{\hookrightarrow} F\}] \hookrightarrow \mathbb{Q}[\{k(X) \stackrel{/ k}{\hookrightarrow} F\}]$. С другой стороны, если $X=Z \times \mathbb{P}^{1}, Y=Z^{\prime} \times \mathbb{P}^{1}$ и $Z^{\prime}$ - двулистное накрытие $Z$, то имеется вложение и в обратном направлении: $\mathbb{Q}[\{k(X) \stackrel{/ k}{\hookrightarrow} F\}] \hookrightarrow \mathbb{Q}[\{k(Y) \stackrel{/ k}{\hookrightarrow} F\}]$. Объединяет $X$ и $Y$ в этом примере то, что их примитивные мотивы (см. ниже) совпадают (и зануляются). Но и это, по-видимому, не очень существенно.

(iii) Из представления $W=\mathbb{Q}[\{k(X) \stackrel{/ k}{\hookrightarrow} F\}]$ можно извлечь размерность $X$ $\left(\operatorname{dim} X=\min \left\{q \geqslant 0 \mid W^{G \mid \bar{L}} \neq 0\right.\right.$, где $\left.\left.\operatorname{deg} \operatorname{tr}(L \mid k)=q\right\}\right)$ и такие "бирациональные мотивные" инварианты "с точностью до изогений", как $\operatorname{Alb}(X), \Gamma\left(X, \Omega_{X \mid k}^{\bullet}\right)($ см. теорему 1.1.10, 3)-5) и предложение 4.1.17.

(iv) Если $W=\mathbb{Q}[X(F)]$, то $\mathbb{Q}[\{k(X) \stackrel{/ k}{\hookrightarrow} F\}]$ - ненулевой фактор $W$ по подмодулю, порожденному всеми $W^{G_{F \mid L}} \mathrm{c} \operatorname{deg} \operatorname{tr}(L \mid k)<q$, при максимально возможном $q(=\operatorname{dim} X)$.

Обозначим через $\mathscr{S} m_{G}(E)$ категорию гладких представлений группы $G$ над полем $E$. Это - полная абелева подкатегория категории всех представлений группы $G$ над полем $E$.

Из топологической простоты группы $G$ (теорема 2.2 .1 ) следует, что в случае $n=\infty$ любое конечномерное гладкое представление $G$ тривиально.

$\mathscr{A} d m$. Рассмотрим теперь более конкретную геометрическую категорию: категорию мотивов.

(Эфбективным) чистым ковариантным мотивом называется пара $(X, \pi)$, состоящая из гладкого проективного многообразия $X$ над $k$ с неприводимыми компонентами $X_{j}$ и проектора $\pi=\pi^{2} \in \bigoplus_{j} B^{\operatorname{dim} X_{j}}\left(X_{j} \times_{k} X_{j}\right)$ в алгебре соответствий на $X$ по модулю численной эквивалентности. Морфизмы определены формулой $\operatorname{Hom}\left(\left(X^{\prime}, \pi^{\prime}\right),(X, \pi)\right)=\bigoplus_{i, j} \pi_{j} B^{\operatorname{dim} X_{j}}\left(X_{j} \times_{k} X_{i}^{\prime}\right) \pi_{i}^{\prime}$. Категория чистых ковариантных мотивов имеет аддитивную и тензорную структуры:

$\left(X^{\prime}, \pi^{\prime}\right) \oplus(X, \pi):=\left(X^{\prime} \coprod X, \pi^{\prime} \oplus \pi\right), \quad\left(X^{\prime}, \pi^{\prime}\right) \otimes(X, \pi):=\left(X^{\prime} \times_{k} X, \pi^{\prime} \times_{k} \pi\right)$.

Примитивным $q$-мотивом называется такая пара $(X, \pi)$ как выше, где $\operatorname{dim} X=q$ и $\operatorname{Hom}\left(Y \times \mathbb{P}^{1},(X, \pi)\right):=\pi \cdot B^{q}\left(X \times_{k} Y \times \mathbb{P}^{1}\right)=0$ для любого гладкого проективного многообразия $Y$ над $k$ размерности $<q$. Например, из теоремы Лефшеца о $(1,1)$-классах следует, что категория чистых примитивных 1-мотивов эквивалентна категории абелевых многообразий над $k$, морфизмы которой тензорно умножены на $\mathbb{Q}$. Как показал У. Яннсен в [6], категория чистых мотивов абелева и полупроста. Из этого результата вытекает, что любой чистый мотив допускает “примитивное" разложение $\bigoplus_{i, j} M_{i j} \otimes \mathbb{L}^{\otimes i}$, где $M_{i j}$ - примитивный $j$-мотив и $\mathbb{L}=\left(\mathbb{P}^{1}, \mathbb{P}^{1} \times\{0\}\right)$ - мотив Лефшеца (см. замечание 4.3.11).

ЗАмЕчАниЕ 1.1.2. Заменив численную эквивалентность произвольным адекватным отношением эквивалентности, мы получим псевдоабелеву тензорную категорию ковариантных мотивов Гротендика. 
ОПРЕДЕЛЕНИЕ 1.1.3. Представление вполне несвязной группы $H$ называется допустимым, если оно - гладкое и неподвижные подпространства всех открытых подгрупп $H$ конечномерны.

Обозначим через $\mathscr{A} d m=\mathscr{A} d m_{G}(\mathbb{Q})$ категорию допустимых представлений группы $G$ над $\mathbb{Q}$.

Заметим, что допустимые представления могут быть бесконечными прямыми суммами. Например, пусть $\left\{A_{\alpha}\right\}$ - набор попарно неизогенных простых абелевых многообразий над $k$. Тогда представление $\bigoplus_{\alpha}\left(A_{\alpha}(F) / A_{\alpha}(k)\right)$ группы $G$ допустимо.

Теорема 1.1.4 [7]. $\mathscr{A} d m$ - подкатегория Серра категории $\mathscr{S} m_{G}$ гладких представлений $G$ над $\mathbb{Q}$.

Другими словами, категория $\mathscr{A} d m$ абелева, замкнута относительно взятия подфакторов (в случае $n=\infty$ именно в этом заключается нетривиальность утверждения) в категории представлений $G$ и относительно расширений в категории гладких представлений $G$. Это показано в [7; следствие 6.5$]$ с помощью вложения $\mathscr{A} d m$ в бо́льшую категорию $\mathscr{I}_{G}$, описанную ниже.

Теорема 1.1.5 [7]. Существует вполне строгий функтор $\mathbb{B}^{\bullet}$ при $n=\infty$ :

$$
\mathscr{M}_{k}:=\left\{\begin{array}{c}
\text { чистые ковариантные } \\
k \text {-мотивы }
\end{array}\right\} \stackrel{\mathbb{B}^{\bullet}}{\longrightarrow}\left\{\begin{array}{c}
\text { градуированные полупростые } \\
\text { допустимые представления } G \\
\text { над } \mathbb{Q} \text { конечной длины }
\end{array}\right\} .
$$

Градуировка соответствует степеням мотива $\mathbb{L}$ в "примитивном" разложении выше.

Грубо говоря, функтор $\mathbb{B}^{\bullet}=\bigoplus_{j}^{\text {graded }} \mathbb{B}^{[j]}$ определяется как пространство 0 -циклов над $F$ по модулю “численной эквивалентности над $k$ ". Точнее, $\mathbb{B}^{\bullet}=$ $\bigoplus_{j}^{\text {graded }} \underset{L}{\lim } \operatorname{Hom}\left([L]^{\text {prim }} \otimes \mathbb{L}^{\otimes j},-\right)$ - градуированная прямая сумма пропредставимых функторов. Здесь $L$ пробегает все подполя в $F$ конечного типа над $k$ и $[L]^{\text {prim }}-$ фактор мотива любой гладкой проективной модели $L$ над $k$ по сумме всех подмотивов вида $M \otimes \mathbb{L}$ для всех эффективных мотивов $M$.

Таким образом, категория $\mathscr{M}_{k}$ становится полной подкатегорией категории градуированных полупростых допустимых представлений $G$ над $\mathbb{Q}$ конечной длины.

ПримеРы 1.1.6. Мотив точки $\operatorname{Spec}(k)$ переходит в тривиальное представление $\mathbb{Q}$ в степени 0. Мотив гладкой собственной кривой $C$ над $k$ переходит в $\mathbb{Q} \oplus J_{C}(F) / J_{C}(F) \oplus \mathbb{Q}[1]$, где $J_{C}$ - якобиан $C$ и $\mathbb{Q}[1]$ обозначает тривиальное представление в степени 1 .

ГиПотеЗА 1.1.7. Функтор $\mathbb{B}^{\bullet}$ задает эквивалентность категорий.

Разумеется, было бы интереснее описать аналогичным образом абелеву категорию $\mathcal{M M}$ смешанных мотивов над $k$, полупростые объекты которой - чистые. Это - еще одно основание для изучения категории $\mathscr{A} d m$ допустимых представлений $G$. 
ПРЕДЛОЖЕНИЕ 1.1 .8 [7]. Если $n=\infty$, то для любого $W \in \mathscr{A} d m$ и любого абелева многообразия $A$ над $k$

$$
\begin{aligned}
& \operatorname{Ext}_{\mathscr{A d m}}^{>0}(\mathbb{Q}, W)=0, \\
& \operatorname{Ext}_{\mathscr{A} d m}^{1}(A(F) / A(k), W)=\frac{\operatorname{Hom}_{\mathbb{Z}}\left(A(k), W^{G}\right)}{\operatorname{Hom}_{G}\left(A(F) / A(k), W / W^{G}\right)}, \\
& \operatorname{Ext}_{\mathscr{A} d m}^{\geqslant 2}(A(F) / A(k), W)=0 .
\end{aligned}
$$

Эти три формулы следует сравнить со следующими гипотетическими тождествами: для любого эффективного мотива $M$

$$
\begin{aligned}
& \operatorname{Ext}_{\mathcal{M M}}^{>0}(\mathbb{Q}, M)=0, \\
& \operatorname{Ext}_{\mathcal{M M}}^{1}\left(H^{1}(A), M\right)=\frac{A(k) \otimes W_{0} M}{\operatorname{Hom}_{\mathcal{M M}}\left(H^{1}(A), M / W_{0} M\right)}, \\
& \operatorname{Ext}_{\mathcal{M} \mathcal{M}}^{\geqslant 2}\left(H^{1}(A), M\right)=0 .
\end{aligned}
$$

Поскольку $A(F) / A(k)$ - каноническое прямое " $H_{1}$ "-слагаемое представления $\mathbb{B}^{\bullet}(A)$, мы видим, что допустимые представления конечной длины должны быть связаны с эффективными мотивами. По крайней мере, Ext-группы между некоторыми неприводимыми объектами двойственны.

$\mathscr{I}_{G}$. Формальные свойства категории $\mathscr{A} d m$ оставляют желать лучшего. Например, чтобы доказать теорему 1.1.4 и предложение 1.1.8, а также предъявить аргумент в пользу гипотезы 1.1 .7 , используется включение $\mathscr{A} d m$ в бо́льшую полную подкатегорию в категории гладких представлений $G$.

ОПРЕДЕЛЕНИЕ 1.1.9. Объект $W \in \mathscr{S} m_{G}(E)$ называется "гомотопически инвариантным" (в бирациональном смысле; происхождение термина объясняется в п. 3.4), если $W^{G_{F \mid L}}=W^{G_{F \mid L^{\prime}}}$ для любого чисто трансцендентного подрасширения $L^{\prime} \mid L$ в $F \mid k$. Полная подкатегория в $\mathscr{S} m_{G}(E)$ с "гомотопически инвариантными" объектами обозначается $\mathscr{I}_{G}(E)$. (Если ограничиться лишь теми $L^{\prime}$, для которых $L^{\prime} \mid k$ - расширение конечного типа, то результат будет тем же, см. [7; следствие 6.2].)

Типичным объектом категории $\mathscr{I}_{G}$ является $\mathbb{Q}$-векторное пространство $C H^{q}\left(X_{F}\right)_{\mathbb{Q}}$ циклов коразмерности $q \geqslant 0$ на схеме $X \times_{k} F$ по модулю рациональной эквивалентности для любого гладкого многообразия $X$ над $k$. (Это следует из свойства спуска: $\left.C H^{*}\left(X_{F}\right)_{\mathbb{Q}}^{G_{F \mid L}}=C H^{*}\left(X_{L}\right)_{\mathbb{Q}} \cdot\right)$

С другой стороны, если $\widetilde{F}$ - алгебраическое замыкание $F(t)$ в некотором алгебраическом расширении поля $F((t))$, то, вообще говоря, $C H^{*}\left(X_{\widetilde{F}}\right)_{\mathbb{Q}} \notin \mathscr{I}_{G}$, так как $\widetilde{F}^{G_{F \mid L}}(x) \varsubsetneqq \widetilde{F}^{G_{F \mid L(x)}}$ при $x \notin \bar{L}$. Например, если $X$ - эллиптическая кривая $u^{2}=P(v)$, то $k(x)((t)) \ni P(x t)^{1 / 2} \notin k((t))(x)$ и $\left[u \mapsto P(x t)^{1 / 2}, v \mapsto x t\right] \in$ $C H_{0}\left(X_{k((x t))}\right) \subseteq C H_{0}\left(X_{k(x)((t))}\right)$, но $\notin C H_{0}\left(X_{\overline{k(t)}}\right)_{\mathbb{Q}}$.

ТеОРема 1.1.10 ([7], $n=\infty)$. Справедливы следующие утверждения.

1) $\mathscr{I}_{G}(E)$ - подкатегория Серра в $\mathscr{S} m_{G}(E)$ (теорема 6.15 в [7]).

2) $\mathscr{A d m}(E) \subset \mathscr{I}_{G}(E)$, т.е. допустимые представления $G$ "гомотопически инвариантны". 
3) Функтор включения $\mathscr{I}_{G}(E) \hookrightarrow \mathscr{S}_{G}(E)$ допускает левый и правый сопряженные функторы $\mathscr{I},-{ }^{(0)}: \mathscr{S}_{G}(E) \rightarrow \mathscr{I}_{G}(E)$ - универсальные факторобъект и подобгект в $\mathscr{I}_{G}(E)$.

4) Объекты $C_{k(X)}:=\mathscr{I} \mathbb{Q}[\{k(X) \stackrel{/ k}{\hookrightarrow} F\}]$ для всех бирачиональных классов $X$ неприводимых многообразий над $k$ задают систему проективных образующих категории $\mathscr{I}_{G}$.

5) Для любого гладкого собственного многообразия $X$ над $k$ имеется каноническая фильтрачия $C_{k(X)} \supset \mathscr{F}^{1} \supset \mathscr{F}^{2} \supset \cdots$, канонические изоморфизмы $C_{k(X)} / \mathscr{F}^{1}=\mathbb{Q}$ и $\mathscr{F}^{1} / \mathscr{F}^{2}=\operatorname{Alb}\left(X_{F}\right)_{\mathbb{Q}}$ и неканоническое расщепление $C_{k(X)} \cong$ $\mathbb{Q} \oplus \operatorname{Alb}\left(X_{F}\right)_{\mathbb{Q}} \oplus \mathscr{F}^{2}$. Член $\mathscr{F}^{2}$ этой фильтрации определен однозначно этими изоморфизмами и условием, что $\operatorname{Hom}_{G}\left(\mathscr{F}^{2}, \mathbb{Q}\right)=0$ u $\operatorname{Hom}_{G}\left(\mathscr{F}^{2}, A(F) / A(k)\right)=0$ для любого абелева многообразия $A$ над $k$. Здесь $\mathrm{Alb}$ - многообразие Альбанезе. (Следствие 6.24 в [7].)

6) Для любого гладкого собственного многообразия $X$ над $k$ имеется каноническая сюргекиия $C_{k(X)} \rightarrow C H_{0}\left(X_{F}\right)_{\mathbb{Q}}$, ингективная, если $X$ унирачионально над кривой (и в некоторых других случалх, когда "известна" $\mathrm{CH}_{0}(X)$, например, если $X$ - фактор четномерной гиперповерхности Ферма степени $\operatorname{dim} X+2$ или $\operatorname{dim} X+3$, для которого $C H_{0}(X)$ - ииклическая).

7) $B \mathscr{I}_{G}(E)$ существуют (ко-) предель.

Две фильтрации. Для представления $M$ группы $G$ определим $N_{j} M$ как подпространство, натянутое на инварианты $M^{G_{F \mid F_{j}}}$ для всех подполей $F_{j} \subseteq F$ степени трансцендентности $j$ над $k$. С точки зрения п. $3.4, N_{j}$ на гладких $G$-модулях - это “то, что приходит из размерности $\leqslant j$ ”. Ясно, что фильтрация "уровня" $N_{\bullet}$ - возрастающая и функториальная.

Тогда член $\mathscr{F}^{j} W$ функториальной убывающей фильтрации $\mathscr{F} \bullet$ на объекте $W$ категории $\mathscr{I}_{G}$ определяется как пересечение ядер всех $G$-гомоморфизмов $\varphi$ из $W$ в объекты $W^{\prime} \in \mathscr{I}_{G}$ уровня $j$, т. е. такие, что $W^{\prime}=N_{j} W^{\prime}$. Если $W=N_{q} W$, то $\mathscr{F}^{q+1} W=0$, поскольку $\operatorname{ker}(W \stackrel{\mathrm{id}}{\longrightarrow} W)=0$.

По-видимому, в случае $G$-модулей вида $C H_{0}\left(X_{F}\right)_{\mathbb{Q}}$ для гладких собственных многообразий $X$ над $k$ это - мотивная фильтрация (см. [8] и [9; лекция 7]), что согласуется с теоремой 1.1.10, 5).

Следующие две гипотезы связывают мотивы Чжоу и кэлеровы дифференциалы (точнее, голоморфную часть когомологий де Рама, см. предложение 4.1.17 ниже) через категорию $\mathscr{I}_{G}$.

ГиПОТЕЗА 1.1.11 [7]. Для любого гладкого собственного неприводимого многообразия $X$ над $k$ естественная сюргекиия $C_{k(X)} \rightarrow C H_{0}\left(X \times_{k} F\right)_{\mathbb{Q}}-$ изоморбизм при $n=\infty$.

Фильтрация $\mathscr{F}^{\bullet}$ на $C_{k(X)}$ совпадает с мотивной фильтрацией на группах Чжоу 0-циклов.

ЗАмечАниЕ 1.1.12. (i) Из теоремы 1.1.10, 6) можно вывести описание категории абелевых многообразий над $k$ с группами морфизмов, тензорно домноженными на $\mathbb{Q}$, как полной подкатегории категории $\mathscr{A} d m_{G} \subset \mathscr{I}_{G}$ в терминах фильтрации “уровня” $N$ • на гладких $G$-модулях. 
(ii) Из гипотезы Блоха и Бейлинсона [8], [9; лекция 7] о мотивной фильтрации на группах Чжоу и из “стандартной” гипотезы Гротендика о полупростоте (утверждающей, что численная и гомологическая эквивалентности совпадают на гладких собственных многообразиях) вытекает, что "численная" эквивалентность совпадает с рациональной на циклах на спектре тензорного произведения двух полей над общим подполем. Точнее, что для любых гладких собственных $k$-многообразий $X$ и $Y$ сюръективный гомоморфизм локализации $C H^{*}\left(X \times_{k} Y\right) \rightarrow C H^{*}\left(k(X) \otimes_{k} k(Y)\right)_{\mathbb{Q}}$ “убивает" численно тривиальные циклы (см. [10; п. 1.4] или [11; предложение 1.1.1]), или, эквивалентно, что $C H^{j}\left(k(X) \otimes_{k} F\right)_{\mathbb{Q}}$ совпадает с $\mathfrak{B}^{j}(M):=\mathbb{B}^{[0]}(M)$, где $M$ - максимальный примитивный $j$-подмотив мотива $\left(X, \Delta_{X}\right)$.

В сочетании с первой частью гипотезы 1.1.11, из этого следовало бы при $n=\infty$, что

(а) $\mathbb{B}^{\bullet}$ - эквивалентность категорий (гипотеза 1.1.7), см. также следствие 1.1.14 ниже;

(б) любой неприводимый объект $\mathscr{I}_{G}$ допустим;

(в) $G$-модули $\operatorname{gr}_{j}^{N} W$ полупросты для любого $W \in \mathscr{I}_{G}$ (гипотеза 4.1.7), где $N$ • определена выше.

Действительно, для некоторого набора гладких проективных $j$-мерных многообразий $Y$ над $k$ имеется сюръективный морфизм $\bigoplus_{Y} \mathbb{Q}[\{k(Y) \stackrel{/ k}{\hookrightarrow} F\}] \stackrel{\xi}{\rightarrow}$ $\operatorname{gr}_{j}^{N} W$, который пропускается через $\bigoplus_{Y} \operatorname{gr}_{j}^{N} C_{k(Y)}$, см. предложение 4.1.3. Если $C_{k(Y)}=C H_{0}\left(Y \times_{k} F\right)_{\mathbb{Q}}$, то $\operatorname{gr}_{j}^{N} C_{k(Y)}=C H^{j}\left(k(Y) \otimes_{k} F\right)_{\mathbb{Q}}$, так что $\xi$ пропускается через $\bigoplus_{Y} C H^{j}\left(k(Y) \otimes_{k} F\right)_{\mathbb{Q}}$.

Наконец, из полупростоты следует, что существуют проекторы $\pi_{Y}$ и изоморфизм $\bigoplus_{Y} \mathfrak{B}^{j}\left(\left(Y, \pi_{Y}\right)\right) \stackrel{\sim}{\longrightarrow} \operatorname{gr}_{j}^{N} W$. Это доказывает (в), а взяв неприводимое $W$ (которое совпадает с $\operatorname{gr}_{j}^{N} W$ при некотором $j$ ), получим также (а) и (б).

(iii) Для любых $W_{1}, W_{2} \in \mathscr{S} m_{G}(\mathbb{Q})$ положим $W_{1} \otimes \mathscr{I} W_{2}:=\mathscr{I}\left(W_{1} \otimes W_{2}\right)$. Как показывает пример $W_{1}=W_{2}=\mathbb{Q}[F \backslash k], W_{3}=\mathbb{Q}$, эта бинарная операция не ассоциативна на $\mathscr{S} m_{G}$.

Из первой части гипотезы 1.1.11 следует "формула Кюннета" - канонический изоморфизм $C_{k}\left(X \times{ }_{k} Y\right) \stackrel{\sim}{\longrightarrow} C_{k(X)} \otimes \mathscr{I} C_{k(Y)}$ для любых гладких неприводимых $k$-многообразий $X, Y$. В п. 4.2 содержатся некоторые доводы в пользу этого "следствия", которые доказывают "формулу Кюннета" в случае, когда $X$ кривая.

Из "формулы Кюннета" следовало бы, что ограничение $\otimes_{\mathscr{I}}$ на $\mathscr{I}_{G}$ - коммутативная и ассоциативная тензорная структура на $\mathscr{I}_{G}$ (согласованная с внутренним функтором $\mathscr{H}$ om, см. замечание 3.0.2, в) и предложение 4.1.14) и что класс проективных объектов стабилен относительно $\otimes \mathscr{g}$.

Было бы интересно найти “полупростой градуированный” вариант $\otimes \mathscr{I}$, с тем чтобы сделать $\mathbb{B}^{\bullet}$ тензорным функтором.

ГиПотеЗА 1.1 .13 [12]. Любой неприводимый обвект $\mathscr{I}_{G}$ содержится в алгебре $\Omega_{F \mid k}^{\bullet}$, если $n=\infty$ и $k$ алгебраически замкнуто. 
СЛЕДСТВИЕ 1.1.14 ([12], $n=\infty)$. Предположим, что гипотеза 1.1 .13 верна. Тогда:

1) любой неприводимый обгект категории $\mathscr{I}_{G}$ допустим; другими словами, " $\mathscr{I}_{G} \approx \mathscr{A} d m$ ";

2) если численная эквивалентность совпадает с гомологической, то $\mathbb{B}^{\bullet}-$ эквивалентность категорий.

ДокАЗАТЕЛЬство. Пусть $W$ - неприводимый объект $\mathscr{I}_{G}$. Найдутся гладкое проективное многообразие $Y$ над $k$ и сюръекция $C_{k(Y)} \rightarrow W$. Если предположить справедливость гипотезы 1.1.13, то представление $W$ вкладывается в $\Omega_{F \mid k}^{q}$ для некоторого целого $q \geqslant 0$. Поскольку из предложения 4.1.17 следует, что $\operatorname{Hom}_{G}\left(C_{k(Y)}, \Omega_{F \mid k}^{q}\right)=\Gamma\left(Y, \Omega_{Y \mid k}^{q}\right)$, то любой гомоморфизм $C_{k(Y)} \rightarrow \Omega_{F \mid k}^{q}$ пропускается через $A^{\operatorname{dim} Y}\left(Y_{F}\right)$, где $A^{*}$ - пространство циклов “по модулю гомологической эквивалентности (де Рама) над $k$ ". Точнее, $A^{\operatorname{dim} Y}\left(Y_{F}\right)$ - образ пространства $C H_{0}\left(Y_{F}\right)_{\mathbb{Q}}$ в $H_{\mathrm{dR} / k}^{2 \operatorname{dim} Y}\left(Y_{F}\right)$. Из конечномерности сингулярных когомологий гладких комплексных многообразий следует допустимость $A^{\operatorname{dim} Y}\left(Y_{F}\right)$, а значит, и допустимость его фактора $W$.

Чтобы установить, что $\mathbb{B}^{\bullet}$ - эквивалентность категорий, достаточно показать, что любое неприводимое допустимое представление $W$ группы $G$ - компонента степени нуль в $\mathbb{B}^{\bullet}(M)$ для некоторого мотива $M$. Поскольку $W$ - фактор $A^{\operatorname{dim} Y}\left(Y_{F}\right)$, это следует из того факта, что $A^{\operatorname{dim} Y}\left(Y_{F}\right)$ совпадает с компонентой степени нуль в $\mathbb{B}^{\bullet}(Y)$, если численная эквивалентность совпадает с гомологической.

Гипотеза 1.1.13 - одна из основных мотивировок для изучения полулинейных представлений $G$ (см. п. 1.2 ниже). Она имеет также следующее геометрическое следствие, высказанное в качестве гипотезы С. Блохом [9; лекция 7].

СЛЕДСТВИЕ 1.1.15 [12]. Пусть гипотеза 1.1 .13 верна. Если $\Gamma\left(X, \Omega_{X \mid k}^{\geqslant 2}\right)=0$ для гладкого собственного многообразия $X$ над $k$, то отображение Алъбанезе индуиирует изоморфизм $C H_{0}(X)^{0} \stackrel{\sim}{\longrightarrow} \mathrm{Alb}(X)$. В этом случае $C_{k(X)}=$ $C H_{0}\left(X_{F}\right)_{\mathbb{Q}}$. (Обратное утверждение общеизвестно, см. [13], [14; теорема 3].)

ДокАЗАТЕЛЬСтво. Согласно теореме $1.1 .10,5), \mathscr{F}^{2} C_{k(X)}$ отщепляется от циклического $G$-модуля $C_{k(X)}: C_{k(X)} \cong \mathbb{Q} \oplus \operatorname{Alb} X(F)_{\mathbb{Q}} \oplus \mathscr{F}^{2} C_{k(X)}$, где Alb многообразие Альбанезе. Так что если $G$-модуль $\mathscr{F}^{2} C_{k(X)}$ - ненулевой, то он цикличен и, следовательно, имеет ненулевой неприводимый фактор $W \in \mathscr{I}_{G}$. Из гипотезы 1.1.13 следует, что существует вложение $W \hookrightarrow \Omega_{F \mid k}^{q}$ для некоторого целого $q \geqslant 0$.

Однако из предложения 4.1.17 следует, что $\operatorname{Hom}_{G}\left(C_{k(X)}, \Omega_{F \mid k}^{\bullet}\right)=\Gamma\left(X, \Omega_{X \mid k}^{\bullet}\right)$, и, значит, $\operatorname{Hom}_{G}\left(C_{k(X)}, \Omega_{F \mid k}^{q}\right)=\operatorname{Hom}_{G}\left(\mathbb{Q} \oplus \operatorname{Alb} X(F)_{\mathbb{Q}}, \Omega_{F \mid k}^{q}\right)$, если $q \leqslant 1$, так что $q \geqslant 2$. Это означает, что $\operatorname{Hom}_{G}\left(C_{k(X)}, \Omega_{F \mid k}^{q}\right)=\Gamma\left(X, \Omega_{X \mid k}^{q}\right)$ - ненулевое для некоторого $q \geqslant 2$.

$\Phi_{G}$ и когомологии гладких представлений. Как объяснялось в п. 1.1, (по крайней мере некоторые) неприводимые допустимые представления соответствуют неприводимым чистым мотивам и Еxt-группы между определенными неприводимыми допустимыми представлениями двойственны к ожидаемым 
значениям Ext-групп между соответствующими чистыми мотивами. В связи с этим возникают такие проблемы, как

- найти другие группы $\operatorname{Ext}_{\mathscr{A} d m}^{*}$ и $\operatorname{Ext}_{\mathscr{S} m_{G}}^{*}$ и сравнить их с гипотетическими значениями соответствующих групп Ext ${ }_{\mathcal{M M}}^{*}$;

- расширить $\mathscr{A} d m$ (или $\mathscr{I}_{G}$ ) и связать полученную бо́льшую категорию $\Psi$ с категорией эфбективных смешанных мотивов таким образом, чтобы, в частности, $\Psi$ содержала такие (не допустимые) объекты, как $F^{\times} / k^{\times}$ (поскольку $\operatorname{Ext}_{\mathscr{S} m_{G}}^{1}\left(F^{\times} / k^{\times}, \mathbb{Q}\right)=\operatorname{Hom}\left(k^{\times}, \mathbb{Q}\right)$, см. предложение 1.1.16, объект $F^{\times} / k^{\times}$играет роль мотива Тэйта), и чтобы объект $\mathbb{Q}$ был попрежнему проективным.

Если возможно описать абелеву категорию $\mathcal{M} \mathcal{M}_{k}$ способом, аналогичным гипотезе 1.1.7 (или хотя бы теореме 1.1.5), то для этого, видимо, нужно рассматривать категорию гладких $G$-модулей конечной длины без подфакторов определенного вида (например, изоморфных $F / k$ ).

Мы увидим, однако (после предложения 1.1.18), что

$$
\operatorname{Ext}_{\mathscr{S} m_{G}}^{1}\left(A(F) / A(k), F^{\times} / k^{\times}\right) \neq 0
$$

для любого абелева многообразия $A$ над $k$. Поэтому рассмотрение весов показывает, что в любом случае связь между $\mathcal{M} \mathcal{M}_{k}$ и $\mathscr{S} m_{G}$ не может быть слишком прямой.

В качестве "верхней оценки" для $\Psi$ можно взять следующую полную аддитивную подкатегорию $\Phi_{G}$ в $\mathscr{S} m_{G}$. Пусть $\mathscr{G} l$ - категория гладких $k$-многообразий, морфизмы которой - композиции гладких морфизмов и замкнутых вложений вида $X \hookrightarrow X \times Y$, задаваемых $k$-точками $Y$. Объекты $\Phi_{G}$ - пределы вида $\mathscr{F}(F):=\lim _{A \longrightarrow} \mathscr{F}(\operatorname{Spec}(A))$, где $\mathscr{F}$ - функтор на $\mathscr{G} l$ и $A$ пробегает конечно порожденные гладкие $k$-подалгебры $F$. Примеры объектов $\Phi_{G}$ включают $\otimes_{F}^{\bullet} \Omega_{F \mid k}^{1}$, $A(F)_{\mathbb{Q}}$ для любой коммутативной $k$-группы $A$ и $\mathbb{Q}[\{L \stackrel{/ k}{\hookrightarrow} F\}]$ для любого $L \mid k$ конечного типа, но не включают $\mathbb{Q}[\{L \stackrel{/ k}{\hookrightarrow} F\}]^{\circ}:=\operatorname{ker}[\mathbb{Q}[\{L \stackrel{/ k}{\hookrightarrow} F\}] \stackrel{\operatorname{deg}}{\longrightarrow} \mathbb{Q}]$.

ПРЕДЛОЖЕНИЕ 1.1.16 [7; предложения 5.1, 5.2]. Пусть $n=\infty u A-$ непрuводимая коммутативная алгебрачческая группа над $k$.

Тогда $\operatorname{Ext}_{\mathscr{S}_{m_{G}}}^{1}\left(A(F)_{\mathbb{Q}}, \mathbb{Q}\right)=0 u \operatorname{Ext}_{\mathscr{S}_{m_{G}}}^{1}(A(F) / A(k), \mathbb{Q})=\operatorname{Hom}(A(k), \mathbb{Q})$.

Можно предположить, что $\operatorname{Ext}_{\mathscr{S}_{m_{G}}}^{*}\left(W_{1}, W_{2}\right)=\operatorname{Ext}_{\mathscr{I}_{G}}^{*}\left(W_{1}, W_{2}\right)$ для любых $W_{1}, W_{2} \in \mathscr{I}_{G}$, если $n=\infty$. При $* \leqslant 1$ это следует из теоремы $\left.1.1 .10,1\right)$. Если $W_{1}, W_{2} \in \mathscr{I}_{G}^{q}$ и $W_{1}$ проективен в $\mathscr{I}_{G}^{q}$, то из леммы 4.1.1 можно получить $\operatorname{Ext}_{\mathscr{S}_{m_{G}}}^{1}\left(W_{1}, W_{2}\right)=\operatorname{Ext}_{\mathscr{I}_{G}}^{1}\left(W_{1}, W_{2}\right)=\operatorname{Ext}_{\mathscr{I}_{G}^{q}}^{1}\left(W_{1}, W_{2}\right)=0$.

В [15] установлена ацикличность в $\mathscr{S} m_{G}$ объектов подкатегорий $\Phi_{G}$ и $\mathscr{I}_{G}$. В доказательстве используется интерпретация гладких представлений как пучков в доминантной топологии на $k$, а их когомологий - как когомологий Чеха. Подробности см. в п. 3.4. Условия ацикличности имеют также приложения к критериям неприводимости представлений $G$.

Чтобы взглянуть на гладкое представление группы $G$ "более геометрически", хотелось бы, чтобы оно соответствовало "более геометрическому" пучку, например пучку в гладкой топологии на $k$. Это возможно, если свойства 
функторов $(-)_{v}$ из предложения 1.2.4 достаточно хороши. (Но, разумеется, получившийся пучок может быть нулевым.) Эти и близкие к ним вопросы обсуждаются в п. 4.4 .

Дифференциальные формы. Чтобы сравнить различные теории когомологий $H^{*}$, можно связать с ними некоторые представления $G$, такие как $H^{*}(F):=\lim H^{*}(U)$, где $U$ пробегает спектры гладких подалгебр $F$, конечно порожденных над $k$, или образ $H_{c}^{*}(F)$ в $H^{*}(F)$ предела $\lim _{\longrightarrow} H^{*}(X)$, где $X$ пробегает гладкие собственные модели подполей $F$ конечного типа над $k$.

Ясно, что $H_{c}^{*}(F)$ - допустимое представление $G$ над $H^{*}(k)$. Из стандартной гипотезы о полупростоте следовало бы, что это представление полупросто. Если $H_{c}^{*}(F)$ полупросто, то в замечании 1.1 .12 , (ii) можно опустить ссылку на стандартную гипотезу о полупростоте.

В случае когомологий де Рама $H^{*}=H_{\mathrm{dR} / k}^{*}$ (убывающая) фильтрация Ходжа на $\Omega_{X \mid k}^{\bullet}$ индуцирует убывающую фильтрацию на $H_{\mathrm{dR} / k, c}^{q}(F) \subset H_{\mathrm{dR} / k}^{q}(F)$ с присоединенными факторами

$$
H_{F \mid k}^{p, q-p}=\underset{\longrightarrow}{\lim } \operatorname{coker}\left[H^{p-1}\left(D, \Omega_{D \mid k}^{q-p-1}\right) \rightarrow H^{p}\left(X, \Omega_{X \mid k}^{q-p}\right)\right],
$$

где $(X, D)$ пробегает множество пар, состоящих из гладкого собственного многообразия $X$ с $k(X) \subset F$ и дивизора с нормальными пересечениями $D$ на $X$ с гладкими неприводимыми компонентами. В частности, $H_{F \mid k}^{q, 0}=\Omega_{F \mid k, \text { рег }}^{q} \subset$ $H_{\mathrm{dR} / k, c}^{q}(F)$ есть $G$-подмодуль, порожденный пространствами $\Gamma\left(X, \Omega_{X \mid k}^{\bullet}\right)$ регулярных дифференциальных форм на всех гладких проективных $k$-многообразиях $X$ с полем функций, вложенным в $F$.

Другой мотивировкой для изучения дифференциальных форм $\Omega_{F \mid k}^{\bullet}$ является вычисление интегралов. Интеграл мероморфной дифференциальной формы $\omega$ на некотором алгебраическом комплексном многообразии $X$ можно вычислять, переводя $\omega$ на другие многообразия при помощи соответствий. В координатах это выглядит как алгебраическая замена переменных. Можно считать, что все поля функций содержатся в некотором общем поле $F$. При этом задача описания свойств (итерированных) интегралов от $\omega\left(\right.$ от $\left.\omega_{1}, \ldots, \omega_{N}\right)$ становится связанной с определением структуры $G$-подмодуля, порожденного $\omega$ (соответственно $\left.\omega_{1} \otimes \cdots \otimes \omega_{N}\right)$, в алгебре кэлеровых дифференциалов $\Omega_{F \mid k}^{\bullet}$ (соответственно в $\Omega_{F \mid k}^{\bullet} \otimes_{k} \cdots \otimes_{k} \Omega_{F \mid k}^{\bullet}$ ).

ПРЕДЛОЖЕНИЕ 1.1.17 [15]. Предположим, что мощность $k$ не превышает континуума. Зафиксируем вложение $\iota: k \hookrightarrow \mathbb{C}$ в поле комплексных чисел. Тогда:

- существуют неканонический $\mathbb{Q}$-линейньй изоморфизм $H_{F \mid k}^{p, q} \cong H_{F \mid k}^{q, p} u$ канонический $\mathbb{C}$-антилинейньй изоморфизм (зависящий от $\iota) H_{F \mid k}^{p, q} \otimes_{k, \iota}$ $\mathbb{C} \cong H_{F \mid k}^{q, p} \otimes_{k, \iota} \mathbb{C}$

- представление $H_{\mathrm{dR} / k, c}^{n}(F)$ (а значит, u $\Omega_{F \mid k, \text { рег }}^{n}$ ) полупросто для любого $1 \leqslant n<\infty$.

Из предложения 4.1.17 следует, что "гомотопически инвариантная" часть представления $\bigotimes_{F}^{\bullet} \Omega_{F \mid k}^{1}$, т. е. его максимальный подобъект в $\mathscr{I}_{G}$, совпадает 
с $\Omega_{F \mid k, \text { рег }}^{\bullet}$ при $n=\infty$. Это еще раз подтверждает когомологическую природу объектов $\mathscr{I}_{G}$, поскольку $\Omega_{F \mid k, \text { рег }}^{\bullet} \subset H_{\mathrm{dR} / k, c}^{q}(F)$.

Приведенные выше примеры $G$-модулей получаются из некоторых (про-) многообразий над $k$ расширением основного поля до $F$. Более общим образом, с каждым бирационально инвариантным функтором $\mathscr{F}$ на некоторой категории многообразий над $k$, или на категории расширений поля $k$ (как в следствии 4.3.7), можно связать $G$-модуль.

Еще два примера $G$-модулей этого вида получаются из бирационально инвариантного функтора Divalg алгебраически тривиальных дивизоров на категории гладких собственных многообразий над $k: \operatorname{Div}_{\mathbb{Q}}^{\circ}=\lim _{U} \operatorname{Div}_{a l g}\left(Y_{U}\right)_{\mathbb{Q}}$, и из функтора Пикара: $\operatorname{Pic}_{\mathbb{Q}}^{\circ}=\lim _{U} \operatorname{Pic}^{\circ}\left(Y_{U}\right)_{\mathbb{Q}}=\operatorname{coker}\left[F^{\times} / k^{\times} \stackrel{\text { div }}{\longrightarrow} \operatorname{Div}_{\mathbb{Q}}^{\circ}\right]$, где $U$ пробегает множество открытых подгрупп вида $G_{F \mid L}$ и $Y_{U}$ - гладкая проективная модель поля $F^{U}=L$ над $k$. Ясно, что

$$
H_{\mathrm{dR} / k, c}^{1}(F)=\operatorname{ker}\left[H_{\mathrm{dR} / k}^{1}(F) \stackrel{\text { Res }}{\longrightarrow} k \otimes \operatorname{Div}_{\mathbb{Q}}^{\circ}\right] .
$$

ПредложениЕ 1.1.18 [7; предложение 3.11]. Пусть $1 \leqslant n \leqslant \infty u A^{\vee}:=$ $\mathrm{Pic}^{\circ} A$ - двойственное абелево многообразие абелева многообразия $A$. Тогда $\mathrm{Pic}_{\mathbb{Q}}^{\circ}=\bigoplus_{A} A^{\vee}(k) \otimes_{\operatorname{End} A}(A(F) / A(k))$, где $A$ пробегает классы изогении простых абелевых многообразий над $k$.

Покажем, что $\operatorname{Ext}_{\mathscr{S} m_{G}}^{1}\left(A(F) / A(k), F^{\times} / k^{\times}\right) \neq 0$ для любого абелева многообразия $A$ над $k$. $G$-модуль $\operatorname{Div}_{\mathbb{Q}}^{\circ}$ входит в точную последовательность $0 \rightarrow$ $F^{\times} / k^{\times} \rightarrow \operatorname{Div}_{\mathbb{Q}}^{\circ} \rightarrow \mathrm{Pic}_{\mathbb{Q}}^{\circ} \rightarrow 0$. Согласно предложению 1.1.18, любой ненулевой элемент $A^{\vee}(k)_{\mathbb{Q}}$ задает вложение $A(F) / A(k)$ в $\mathrm{Pic}_{\mathbb{Q}}^{\circ}$, тем самым индуцируя расширение $A(F) / A(k)$ модулем $F^{\times} / k^{\times}$внутри $\operatorname{Div}_{\mathbb{Q}}^{\circ}$. Чтобы увидеть, что это расширение не расщепляется, заметим, что любая общая $F$-точка $x$ группы $A$, рассматриваемая как элемент $A(F)_{\mathbb{Q}}$, отождествляет пространство $\operatorname{Hom}_{G}\left(A(F)_{\mathbb{Q}}, \operatorname{Div}_{\mathbb{Q}}^{\circ}\right)$ с подпространством в $\left(\operatorname{Div}_{\mathbb{Q}}^{\circ}\right)^{\operatorname{Stab}_{x}}$, элементы которого - это $\mathbb{Q}$-дивизоры на $A$, инвариантные относительно сдвигов на элементы кручения в $A(k)$. Поскольку подгруппа кручения в $A(k)$ плотна по Зарискому, любой такой дивизор - нулевой, т. е. это подпространство - нулевое.

Из очевидным образом модифицированного предложения 1.1 .18 нетрудно

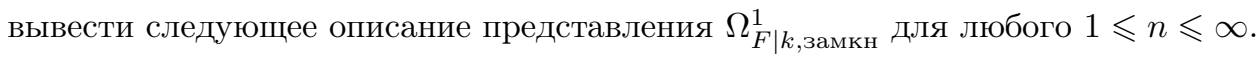

ПРЕДЛОЖЕНИЕ 1.1.19. Пусть $1 \leqslant n \leqslant \infty$.

1) Максимальное полупростое подпредставление группы $G$ в $\Omega_{F \mid k \text {,замкн }}^{1} \kappa a$ нонически изоморфно

$$
\bigoplus_{A} \Gamma\left(A, \Omega_{A \mid k}^{1}\right)^{A(k)} \otimes_{\operatorname{End} A}(A(F) / A(k))=(F / k) \oplus k \otimes\left(F^{\times} / k^{\times}\right) \oplus \Omega_{F \mid k, \text { per }}^{1},
$$

где А пробегает классы изогении простых коммутативных алгебрачческих $k$-гpynn.

2) Максимальное полупростое подпредставление группь $G$ в $H_{\mathrm{dR} / k}^{1}(F) \kappa a-$ нонически изоморфно

$$
\bigoplus_{A} H_{\mathrm{dR} / k}^{1}(A) \otimes_{\mathrm{End} A}(A(F) / A(k))=k \otimes\left(F^{\times} / k^{\times}\right) \oplus H_{\mathrm{dR} / k, c}^{1}(F),
$$


где А пробегает классы изогении простых коммутативных алгебрачческих $k$-групп (с нулевым слагаемым, соответствующим $\mathbb{G}_{a}$ ).

3) Представление $H_{\mathrm{dR} / k}^{1}(F) /\left(k \otimes\left(F^{\times} / k^{\times}\right)\right)$группь $G$ канонически изоморфно прямой сумме

$$
\bigoplus_{A}\left[H_{\mathrm{dR} / k}^{1}(k(A)) /\left(k \otimes\left(k(A)^{\times} / k^{\times}\right)\right)\right] \otimes_{\operatorname{End} A}(A(F) / A(k)),
$$

где A пробегает классы изогении простых абелевых многообразий над $k$.

Это наводит на мысль, что а) классы изоморфизма неприводимых подфакторов $H_{c}^{*}(F)$ - те же, что и у $\Omega_{F \mid k, \text { рег }}^{\bullet}$ б) они естественно отождествляются с неприводимыми эффективными примитивными мотивами и в) классы изоморфизма неприводимых подфакторов $H^{*}(F)$ связаны с более общими неприводимыми эффективными мотивами, такими как мотив Тэйта $\mathbb{Q}(-1)$ в случае $H_{\mathrm{dR} / k}^{1}(F)$.

1.2. От линейных представлений к полулинейным. Представление $\Omega_{F \mid k}^{\bullet}$ группы $G$ имеет также естественную структуру $F$-векторного пространства, снабженного полулинейным действием $G$.

ОПРЕДЕЛЕНИЕ 1.2.1. Пусть $K$ - поле, $H$ - полугруппа эндоморфизмов $K$ и $k=K^{H}$.

Полулинейное представление $H$ над $K$ - это $K$-векторное пространство $V$, снабженное полулинейным действием $H$, т.е. таким аддитивным действием $H \times V \rightarrow V$, что $\sigma(a \cdot v)=\sigma a \cdot \sigma v$ для любых $\sigma \in H, v \in V$ и $a \in K$. Это - то же, что модуль над ассоциативной центральной $k$-алгеброй $K\langle H\rangle:=K \otimes_{\mathbb{Z}} \mathbb{Z}[H]$ с очевидным левым действием $K$ и диагональным левым действием $H$. Будем говорить, что полулинейное представление полугруппы $H$ невырождено, если действие каждого элемента $H$ инъективно. (Если $\operatorname{dim}_{K} V<\infty$, это эквивалентно условию $K \otimes_{\sigma(K)} \sigma(V)=V$.)

Полулинейные представления $H$, конечномерные над $K$, образуют абелеву тензорную $k$-линейную категорию. Эта категория - жесткая, если элементы полугруппы $H$ обратимы. Множество классов изоморфизма невырожденных полулинейных $K$-представлений полугруппы $H$ степени $r$ канонически отождествляется с множеством $H^{1}\left(H, \mathrm{GL}_{r} K\right)$.

Обозначим через $\mathscr{C}$ категорию гладких полулинейных представлений $G$ над $F$.

Как известно после работ Гильберта, Тэйта, Сена, Фонтэна и др., мощным средством изучения представлений Галуа являются полулинейные представления. Мы попытаемся их применить в контексте представлений группы $G$.

Нас интересуют линейные представления $G$, особенно неприводимые, и, в еще большей степени, неприводимые "гомотопически инвариантные" представления, т. е. объекты $\mathscr{I}_{G}$.

Имеются строгие забывающие функторы $\mathscr{S} m_{G} \stackrel{\text { for }}{\longleftarrow} \mathscr{C} \stackrel{\text { for }}{\longleftrightarrow} \mathscr{S} m_{G}(k)$, допускающие левые сопряженные функторы расширения коэффициентов до $F$ : $\mathscr{S} m_{G} \stackrel{\otimes F}{\longrightarrow} \mathscr{C} \stackrel{\otimes_{k} F}{\longleftarrow} \mathscr{S} m_{G}(k)$, где $\mathscr{S} m_{G}(k)$ - категория гладких представлений $G$ над $k$, так что $W \hookrightarrow \operatorname{for}(W \otimes F)$ или $W \hookrightarrow$ for $\left(W \otimes_{k} F\right)$. 
Функтор $F \otimes_{k}$ строг, но не полон. Например, если $U \subset G$ - открытая подгруппа и $\bar{f} \in\left(F^{\times} / k^{\times}\right)^{U} \backslash\{1\}$, то отображение $[\sigma] \mapsto \sigma f \cdot[\sigma]$ определяет элемент $\operatorname{End}_{\mathscr{C}}(F[G / U])$, который не лежит в подпространстве $\operatorname{End} \mathscr{S}_{m_{G}}(k)(k[G / U])$. Другой пример: $k=\operatorname{End}_{\mathscr{S}_{m_{G}}(k)}(F) \varsubsetneqq \operatorname{End}_{\mathscr{C}}\left(F \otimes_{k} F\right) \supseteq k \oplus k$, так как $F \otimes_{k} F=$ $\operatorname{Sym}_{k}^{2} F \oplus \bigwedge_{k}^{2} F$. Однако функтор $\mathscr{I}_{G}(k) \stackrel{F \otimes_{k}}{\longrightarrow} \mathscr{C}$ вполне строгий [16; лемма 0.1], и любой объект $W \in \mathscr{I}_{G}(k)$ восстанавливается по $W \otimes_{k} F \in \mathscr{C}$, см. лемму 1.2.6.

Хотя функтор $F \otimes_{k}$ и не сохраняет неприводимость, для любого неприводимого $W \in \mathscr{S} m_{G}$ объект $W \otimes F \in \mathscr{C}$ допускает неприводимый полулинейный фактор $V$ с включением $W \subset V$.

Таким образом, любой неприводимый объект категории $\mathscr{S} m_{G}$ содержится в неприводимом объекте категории $\mathscr{C}$, и задача описания неприводимых объектов категории $\mathscr{S} m_{G}$ разбивается на описание а) неприводимых объектов $\mathscr{C}$ и б) их линейных подмодулей.

Приведем два довода в пользу того, что $\mathscr{C}$ в некоторых отношениях проще, чем $\mathscr{S} m_{G}$.

- Все представления $A(F) / A(k)$ группы $G$ для всех абелевых многообразий $A$ над $k$ (т. е. соответствующие всем чистым 1-мотивам) содержатся в одном неприводимом объекте $\Omega_{F \mid k}^{1}$ категории $\mathscr{C}$. А именно, любая достаточно общая 1-форма $\eta \in \Gamma\left(A, \Omega_{A \mid k}^{1}\right)$ задает вложение $A(F) / A(k) \hookrightarrow$ $\Omega_{F \mid k}^{1}$, посылая точку $k(A) \stackrel{\sigma}{\hookrightarrow} F$ в $\sigma \eta \in \Omega_{F \mid k}^{1}$.

- Из теоремы 90 Гильберта следует, что категория $\mathscr{C}$ обладает счетной системой циклических образующих: $P_{m}:=F\left[G / G_{F \mid K_{m}}\right]$, где $K_{m}$ - чисто трансцендентное расширение $k$ в $F$ степени $m$.

Вообще говоря, для произвольной группы полулинейных представлений может оказаться "гораздо больше", чем линейных. Например, если группа корней из единицы $\mu_{\infty} \cong \mathbb{Q} / \mathbb{Z}$ действует на $k(t)$ заменами $\zeta: t \mapsto \zeta t$, то пространство одномерных полулинейных представлений

$$
H^{1}\left(\mu_{\infty}, k(t)^{\times}\right)=\left(\lim _{n} k(t)^{\times} / k\left(t^{n}\right)^{\times}\right) / k(t)^{\times}
$$

огромно (и неотделимо в естественной топологии), хотя пространство одномерных $E$-линейных представлений $\operatorname{Hom}\left(\mu_{\infty}, E^{\times}\right) \subseteq \widehat{\mathbb{Z}}$ относительно невелико.

Множество классов изоморфизма неприводимых объектов $\mathscr{C}$ имеет мощность континуума, если $k$ счетно. Действительно, пусть $F^{\prime}$ - алгебраически замкнутое подполе в $F \mid k$ конечной степени трансцендентности. Для каждого одномерного представления $\varphi: G_{F^{\prime} \mid k} \rightarrow k^{\times}$зафиксируем неприводимый фактор $V_{\varphi} \in \mathscr{C}$ представления $F\left[G / G_{F \mid F^{\prime}}\right] \otimes_{k\left[G_{F^{\prime} \mid k}\right]} \varphi$. Заметим, что в $V_{\varphi}^{G_{F \mid F^{\prime}}}$ имеется подпредставление $G_{F^{\prime} \mid k}$, изоморфное $\varphi$. Скажем, что $\varphi \sim \psi$, если $V_{\varphi} \cong V_{\psi}$. Если $\varphi \sim \psi$, то в $V_{\varphi}^{G_{F \mid F^{\prime}}}$ имеется подпредставление $G_{F^{\prime} \mid k}$, изоморфное $\psi$. Поскольку $\left|V_{\varphi}^{G_{F \mid F^{\prime}}}\right|=|k|$, классы эквивалентности имеют мощность $\leqslant|k|$. Заметим, что имеется $\geqslant 2^{|\mathbb{N}|}$ только тех из $\varphi$, которые пропускаются через модуль группы $G_{F^{\prime} \mid k}$. Поэтому если $k$ счетно, то множество классов эквивалентности $\varphi$ имеет мощность $\geqslant 2^{|\mathbb{N}|}$, что и требовалось. 
Верхняя оценка $\leqslant 2^{|k|}$ для множества циклических объектов $\mathscr{C}$ следует из того, что имеется счетное число образующих $P_{m}$ категории $\mathscr{C}$, мощность каждой из которых равна $|k|$.

С другой стороны, мне не известно никакой процедуры, которая бы могла выдать настолько много неприводимых объектов $\mathscr{C}$, хотя бы гипотетически. Поэтому было бы естественно ограничиться какой-нибудь “относительно небольшой" полной подкатегорией в $\mathscr{C}$. (Например, объекты которой порождаются как $F$-векторное пространство подпредставлениями из $\mathscr{I}_{G}(k)$. Ниже приводится другое, хотя и более слабое, но и чуть более явное условие "глобальной порожденности" на полулинейные факторы $W \otimes F$ для $W \in \mathscr{I}_{G}$.)

Однако категория $\mathscr{C}$ "проста" в том смысле, что не имеет нетривиальных собственных подкатегорий, замкнутых относительно прямых произведений и подфакторов в $\mathscr{C}$. Точнее, справедливо следующее утверждение.

Лемма 1.2.2. Аннулятор в $\lim _{U} F[G / U]$ любого обгекта $0 \neq V \in \mathscr{C}-$ нулевой. Для любого иелого $m \geqslant 0$ имеется вложение $P_{m}$ в прямое произведение в $\mathscr{C}$ копий $V$.

ДокАЗАТЕльство. Если $0 \neq \alpha \in{\underset{\lim }{\longleftarrow}}_{U} F[G / U]$ аннулирует $V$, то для любого $L$ конечного типа над $k$ проекция $0 \neq \alpha_{L}=\sum_{i=1}^{N} a_{i} \sigma_{i} \in F\left[G / G_{F \mid L}\right]$ аннулирует $V^{G_{F \mid L}}$. Зафиксируем $L, 0 \neq v \in V^{G_{F \mid L}}$ и функционал $\varphi \in \operatorname{Hom}_{F}(V, F)$ такой, что $\varphi\left(\sigma_{i} v\right) \neq 0$ для всех $1 \leqslant i \leqslant N$. Тогда $\varphi(\alpha(f v))=\sum_{i=1}^{N} a_{i} \varphi\left(\sigma_{i} v\right) \cdot \sigma_{i} f$ обращается в нуль для любого $f \in L^{\times}$, что противоречит теореме Артина о независимости характеров $L^{\times}$.

$\operatorname{Hom}_{\mathscr{C}}\left(P_{m}, V\right)$ - ненулевое $K_{m}$-векторное пространство (по определению, $t \alpha$ : [id $] \mapsto t \cdot \alpha[\mathrm{id}]$ для любого $\left.t \in K_{m}\right)$. Поэтому

$$
\bigcap_{\alpha \in \operatorname{Hom}_{\mathscr{C}}\left(P_{m}, V\right)} \operatorname{ker} \alpha \subseteq \bigcap_{t \in K_{m}^{\times}} \operatorname{ker}\left(P_{m} \stackrel{t \alpha}{\longrightarrow} V\right)=0
$$

для любого $\alpha \neq 0$ в силу линейной независимости характеров $\operatorname{Hom}\left(K_{m}^{\times}, F^{\times}\right)$.

ЗАмечАниЕ 1.2.3. Утверждение леммы 1.2 .2 означает, что центральная $k$-алгебра $\lim _{U} F[G / U]$ топологически проста (ср. с [17], [18] в случае конечных расширений Галуа). Поэтому любая “относительно небольшая" подкатегория $\mathscr{C}$ не может быть "слишком хорошей".

Отныне (до конца этого раздела) будем считать, что $n=\infty$.

Нормирования и ассоциированные функторы [19]. Чтобы сопоставить представлению $G$ функтор на категории $k$-многообразий, можно попытаться "аппроксимировать" кольца их подполями. Очевидно, это не работает буквально, но зато работает в случае колец дискретного нормирования поля $F$.

Пусть $v: F^{\times} / k^{\times} \rightarrow \mathbb{Q}$ - дискретное нормирование ранга один и $\mathscr{O}_{v}-$ кольцо нормирования. Положим $G_{v}:=\left\{\sigma \in G \mid \sigma\left(\mathscr{O}_{v}\right)=\mathscr{O}_{v}\right\}$. Это - максимальная замкнутая, но не открытая подгруппа в $G$.

ПРЕДЛОЖЕНИЕ 1.2.4. Для любого дискретного нормирования $v$ ранга один, тривиалвного на $k$, аддитивный функтор $(-)_{v}: \mathscr{S} m_{G} \rightarrow \mathscr{S} m_{G_{v}}, W \mapsto W_{v}:=$ $\sum_{F^{\prime} \subset \mathscr{O}_{v}} W^{G_{F \mid F^{\prime}}} \subseteq W$, вполне строг и сохраняет сюргекции и ингекиии. 
Тогда аддитивный подфунктор $\Gamma: \mathscr{S} m_{G} \rightarrow \mathscr{S} m_{G}$ тождественного функтора, заданный формулой $W \mapsto \Gamma(W):=\bigcap_{v} W_{v}$, где $v$ пробегает дискретные нормирования $F^{\times} / k^{\times} \rightarrow \mathbb{Q}$, сохраняет инъекции.

Пример 1.2.5. $\quad \Gamma\left(\Omega_{F \mid k}^{1}\right)=\Omega_{F \mid k, \text { рег }}^{1} \cong \bigoplus_{A}(A(F) / A(k)) \otimes_{\text {End } A} \Gamma\left(A, \Omega_{A \mid k}^{1}\right)$, где $A$ пробегает множество классов изогении простых абелевых многообразий над $k,-$ пространство регулярных 1-форм.

Лемма 1.2.6. Если $k=\bar{k}$, то функтор $\mathscr{I}_{G}(k) \stackrel{F \otimes_{k}}{\longrightarrow} \mathscr{C}$ вполне строг. Композичии $\mathscr{I}_{G}(k) \stackrel{\otimes_{k} F}{\longrightarrow} \mathscr{C} \stackrel{\text { Го for }}{\longrightarrow} \mathscr{S} m_{G}(k)$ u $\mathscr{I}_{G} \hookrightarrow \mathscr{S} m_{G} \stackrel{\Gamma}{\longrightarrow} \mathscr{S} m_{G}$ тождественны.

Первая часть - это лемма 0.1 из [16], а вторая часть вытекает из лемм 4.4.7 и 4.4.8.

ЗАМЕчАНИЕ 1.2.7. (i) Для понимания категории $\mathscr{I}_{G}(k)$ основной задачей является описание оболочки Серра абелевой подкатегории $\mathscr{I}_{G} \otimes F$ категории $\mathscr{C}$. Ориентиром для этого может служить гипотеза 1.1.13.

(ii) Из леммы 1.2 .6 следует, что любой полулинейный фактор $V$ объекта $W \otimes_{k} F$ для $W \in \mathscr{I}_{G}$ (в частности, любое неприводимое полулинейное представление $V$, содержащее "гомотопически инвариантное" представление), "глобально порожден", т. е. $\Gamma(V) \otimes F \rightarrow V$ сюръективно.

Это условие можно наложить на класс "интересных" полулинейных представлений. Имеются причины считать, что $(-)_{v}$ точен (см. [19]). Отсюда следовали бы некоторые приятные свойства категории "глобально порожденных" полулинейных представлений.

Допустимые полулинейные представления. Так же, как и изучение линейных представлений группы, изучение полулинейных представлений естественно начинать с конечномерных представлений. Однако из следствия 3.4.8 будет вытекать их тривиальность.

ТеОРема 1.2.8 [7]. Любое конечномерное гладкое полулинейное представление $G$ над F тривиально при $n=\infty$.

ОПРЕДЕЛЕНИЕ 1.2.9. Гладкое полулинейное представление $V$ группы $G$ над $F$ называется допустимым, если для любой открытой подгруппы $U \subseteq G$ неподвижное подпространство $V^{U}$ конечномерно над неподвижным подполем $F^{U}$ (или, эквивалентно, $\operatorname{dim}_{L} V^{G_{F \mid L}}<\infty$ для любого подполя $L \subset F$ конечного типа над $k$ ).

Tеорема 1.2.10 [12], [16]. Обозначим через $\mathscr{A}$ категорию допустимых полулинейных представлений группы $G$ над $F$. Тогда $\mathscr{A}$ - абелева тензорная (но не жесткая) категория.

Функтор $H^{0}\left(G_{F \mid L},-\right)$ точен на $\mathscr{A}$ для любого подполя $L \subseteq F$, так что $F-$ проективный обгект $\mathscr{A}$.

Тензорность категории $\mathscr{A}$ следует из предложения 3.2.2.

Как вытекает из [16; лемма 3.1], в качестве циклических образующих категории $\mathscr{A}$ можно взять факторы $Q_{n}:=F\left[G / U_{n}\right]$ циклических образующих $P_{n}$ 
категории $\mathscr{C}$ для всех $n \gg 0$, поскольку $\operatorname{Hom}_{\mathscr{C}}\left(Q_{n}, V\right)=V_{n}^{U_{n}}$. Здесь $U_{n}$ обозначает прообраз в $G_{\left\{F, K_{n}\right\} \mid k} \subset G$ подгруппы сдвигов в $G_{K_{n} \mid k}$ на элементы кругового поля, т. е. состоящий из преобразований вида $x_{j} \mapsto x_{j}+b_{j}$ для всех $1 \leqslant j \leqslant n$, где $b_{j} \in \mathbb{Q}^{\mathrm{ab}}$.

ПРимеР 1.2.11. Обозначим через $\mathfrak{m}$ ядро гомоморфизма умножения $F \otimes_{k_{0}}$ $F \stackrel{\times}{\longrightarrow} F$, где $k_{0}=k \cap \overline{\mathbb{Q}}-$ числовое подполе поля $k$. Это - идеал в алгебре $F \otimes_{k_{0}} F$, степени $\mathfrak{m}^{s} \subseteq F \otimes_{k_{0}} F$ которого для всех $s \geqslant 0$ мы будем рассматривать как объекты $\mathscr{C}$. Структура $F$-векторного пространства на $\mathfrak{m}^{s}$ задается левым $F$-умножением, т. е. посредством отождествления $F$ с $F \otimes_{\overline{\mathbb{Q}}} \overline{\mathbb{Q}}$.

Заметим, что $\mathfrak{m}^{s} / \mathfrak{m}^{s+1}=\operatorname{Sym}_{F}^{s} \Omega_{F}^{1}$. Поэтому полулинейные представления $\bigwedge_{F}^{\bullet}\left(\mathfrak{m} / \mathfrak{m}^{s}\right)$ и $\bigotimes_{F}^{q}\left(\mathfrak{m} / \mathfrak{m}^{s}\right)$ допустимы для любых $q \geqslant 0$ и $s \geqslant 2$, если степень трансцендентности $k$ конечна, а объекты $\operatorname{Sym}_{F}^{s} \Omega_{F \mid k}^{\bullet}$ допустимы всегда и для любых $s \geqslant 1$.

В случае, когда $k=\overline{\mathbb{Q}}$ - поле алгебраических комплексных чисел, категория $\mathscr{A}$ допускает следующее явное описание.

Для каждых $q \geqslant 0$ и $V \in \mathscr{A}$ пусть $W^{q} V$ - сумма образов всевозможных $\mathscr{C}$-морфизмов в $V$ из $F$-тензорных степеней $\bigotimes_{F}^{\geqslant q} \mathfrak{m}$. Ясно, что $W^{\bullet}-$ функториальная убывающая фильтрация на объектах $\mathscr{A}$ и что эта фильтрация мультипликативна: $\left(W^{p} V_{1}\right) \otimes_{F}\left(W^{q} V_{2}\right) \subseteq W^{p+q}\left(V_{1} \otimes_{F} V_{2}\right)$ для любых $p, q \geqslant 0$ и любых $V_{1}, V_{2} \in \mathscr{A}$.

1) Присоединенные факторы $\operatorname{gr}_{W}^{q}$ фильтрации $W^{\bullet}$ на объектах $\mathscr{A}$ - конечные прямые суммы прямых слагаемых объекта $\bigotimes_{F}^{q} \Omega_{F}^{1}$ (см. [16; теорема 4.10]). В частности, любой объект категории $\mathscr{A}$ допускает неприводимый фактор.

2) Категория $\mathscr{A}$ расщепляется в прямую сумму двух своих полных подкатегорий, одна из которых эквивалентна категории конечномерных $k$-векторных пространств, а вторая - $\mathscr{A}^{\circ}-$ состоит из таких объектов $V$, что $V^{G}=0$ (см. [16; лемма 4.13]).

3) Любой объект $V$ категории $\mathscr{A}^{\circ}$ является фактором прямой суммы объектов (конечной длины) вида $\bigotimes_{F}^{q}\left(\mathfrak{m} / \mathfrak{m}^{s}\right)$ для некоторых $q, s \geqslant 1$ (см. [16; теорема 4.10]).

4) Если $V \in \mathscr{A}$ конечно порожден, то он конечной длины и пространство $\operatorname{Ext}_{\mathscr{A}}^{j}\left(V, V^{\prime}\right)$ конечномерно для любого $j \geqslant 0$ и любого $V^{\prime} \in \mathscr{A}$; если $V \in \mathscr{A}$ неприводим и $\operatorname{Ext}_{\mathscr{A}}^{1}\left(\mathfrak{m} / \mathfrak{m}^{q}, V\right) \neq 0$ для некоторого $q \geqslant 2$, то $V \cong \operatorname{Sym}_{F}^{q} \Omega_{F}^{1}$ и $\operatorname{Ext}_{\mathscr{A}}^{1}\left(\mathfrak{m} / \mathfrak{m}^{q}, V\right) \cong k$ (см. [16; следствие 4.17]).

5) В категории $\mathscr{A}^{\circ}$ нет проективных объектов [16; следствие 4.14], однако $\bigotimes_{F}^{q} \mathfrak{m}$ - ее “проективные прообразующие": функтор $\operatorname{Hom}_{\mathscr{C}}\left(\bigotimes_{F}^{q} \mathfrak{m},-\right)=$ $\underset{\lim }{\longrightarrow} \operatorname{Hom}_{\mathscr{A}}\left(\bigotimes_{F}^{q}\left(\mathfrak{m} / \mathfrak{m}^{N}\right),-\right)$ точен на $\mathscr{A}$ для любого $q$ (см. [16; следствие 4.16]).

Более того, (по крайней мере при $k=\overline{\mathbb{Q}}$ ) имеется функтор $\mathscr{S}$, заданный формулой $V \mapsto\left(Y \mapsto \mathscr{V}_{Y}(Y)\right)$, отждествляющий категорию $\mathscr{A}$ с категорией "когерентных" пучков в гладкой топологии $\mathfrak{S m}_{k}$ на $k$ (см. [16; следствие 5.2]). По определению, $\mathfrak{S m}_{k}$ - категория локально доминантных морфизмов гладких $k$-схем, снабженная предтопологией, в которой покрытия - это сюръективные гладкие морфизмы. Ясно, что замены базы сохраняют покрытия. 
Пучок $\mathscr{O}$-модулей на $\mathfrak{S m}_{k}$ называется “когерентным”, если его ограничение на малый этальный сайт (или, эквивалентно, на малый сайт Зариского) любого гладкого $k$-многообразия когерентно. Здесь $\mathscr{O}$ - структурный предпучок сайта $\mathfrak{S m}_{k}$ (который сопоставляет каждому $Y \in \mathfrak{S m}_{k}$ его $k$-алгебру регулярных функций $\mathscr{O}(Y))$. Ясно, что $\mathscr{O}-$ пучок на $\mathfrak{S m}_{k}$.

Сначала определяются ограничения пучка $\mathscr{S}(V)$ на проективные пространства (они оказываются пучками сечений однородных векторных расслоений), а на произвольные гладкие $k$-многообразия их можно локально продолжить как обратные образы относительно этальных морфизмов в проективные пространства. Зная явный вид объектов $\mathscr{A}$, корректность такого определения проверить нетрудно (см. [16; лемма 5.1]). Таким образом, $\left.\mathscr{S}(V)\right|_{Y}=\mathscr{V}_{Y}-$ локально свободный когерентный пучок на $Y$ с общим слоем $V^{G_{F \mid k(Y)}}$ для каждого $V \in \mathscr{A}$ и любого гладкого $k$-многообразия $Y$. Функториальность следует из того, для любого доминантного морфизма $X \stackrel{\pi}{\rightarrow} Y$ гладких $k$-многообразий включение общих слоев $k(X) \otimes_{k(Y)} V^{G_{F \mid k(Y)}} \subseteq V^{G_{F \mid k(X)}}$ индуцирует вложение когерентных пучков $\pi^{*} \mathscr{V}_{Y} \hookrightarrow \mathscr{V}_{X}$ на $X$.

Особый интерес среди $k$-линейных представлений группы $G$ представляют допустимые представления, образующие полную подкатегорию в $\mathscr{I}_{G}(k)$. Хотя тензорное умножение на $F$ и не переводит их в допустимые полулинейные представления, ${ }^{1}$ существует аналогичный функтор в противоположном направлении $\Gamma: \mathscr{A} \rightarrow \mathscr{S} m_{G}(k)$ - который можно назвать функтором "глобальных сечений", - строгий и точный слева, по крайней мере, если $k=\overline{\mathbb{Q}}$. Функтор $Г$ уже был определен на с. 102, после предложения 1.2.4, причем даже в большей общности. Однако бывает полезно сначала сопоставить гладким представлениям $V$ группы $G$ пучки $\mathscr{V}$ на $\mathfrak{S m}_{k}$, и лишь потом брать глобальные сечения.

Имеется следующий универсальный (но далеко не единственный) способ "глобализации" гладких представлений $G$. Пусть $V \in \mathscr{S} m_{G}, Y$ - неприводимое гладкое $k$-многообразие, $D \in Y^{1}-$ неприводимый дивизор и $v_{D}$ - соответствующее дискретное нормирование поля $k(Y)$. Выберем вложение $k(Y)$ в $F$ над $k$ и продолжение $v$ нормирования $v_{D}$ до дискретного нормирования ранга 1 поля $F$. Пусть $\widetilde{F} \subset F$ - максимальное подполе над $k$ такое, что $v\left(\widetilde{F}^{\times}\right)=v\left(k(Y)^{\times}\right)$. Положим

$$
\mathscr{V}_{Y, D}=V^{G_{F \mid k(Y)}} \cap \bigcap_{\widetilde{F}}\left(\sum_{k \subset F^{\prime} \subset \widetilde{F} \cap \mathscr{O}_{v}} V^{G_{F \mid F^{\prime}}}\right) .
$$

"Когерентные" пучки содержатся в категории $\mathscr{F} l$ плоских (как $\mathscr{O}$-модули) "квазикогерентных" пучков в гладкой топологии. Для любого плоского "квазикогерентного" пучка $\mathscr{V}$ в гладкой топологии $k$-векторное пространство $\Gamma\left(Y, \mathscr{V}_{Y}\right)$ является бирациональным инвариантом гладкого собственного $Y$. Это следует из принципа Гартогса и того, что любое бирациональное отображение - композиция бирационального морфизма и отображения, обратного бирациональному

\footnotetext{
${ }^{1}$ Предположим, что $W \otimes F$ допустимо для некоторого гладкого представления $W$ группы $G$ при $n \leqslant \infty$. Докажем, что $W$ конечномерно. Из включения $(W \otimes F)^{G_{F \mid L}} \supseteq W^{G_{F \mid L}} \otimes L$ следует, что $\operatorname{dim} W^{G \mid L}<\infty$ для любого $L$ конечной степени трансцендентности над $k$. Поэтому $\operatorname{dim} W<\infty$, если $n<\infty$. Если $n=\infty$, то из предложения 3.0 .8 получаем, что $W$ тривиально и, в частности, конечномерно.
} 
морфизму, которое корректно определено вне подмножества коразмерности $\geqslant 2$ (см. [16; лемма 5.3]).

Благодаря бирациональной инвариантности, можно определить точный слева (не строгий) функтор $\mathscr{F} l \stackrel{\Gamma}{\longrightarrow} \mathscr{S} m_{G}(k)$ формулой $\mathscr{V} \mapsto \lim \Gamma\left(Y, \mathscr{V}_{Y}\right)$, где $Y$ пробегает гладкие проективные модели подполей в $F$ конечного типа над $k$. Тогда $\Gamma(V)=\Gamma(\mathscr{V})$. Ограничение $\Gamma$ на подкатегорию "когерентных" пучков строго, поскольку $\Gamma\left(Y^{\prime}, \mathscr{V}_{Y^{\prime}}\right)$ порождает общий слой пучка $\mathscr{V}_{Y^{\prime}}$ для подходящих конечных накрытий $Y^{\prime}$ многообразия $Y$, если $\mathscr{V}$ - “когерентный”.

Если $\Gamma\left(Y, \mathscr{V}_{Y}\right)$ обладает свойством спуска Галуа, то $\Gamma(V)=\Gamma(\mathscr{V})$ допустимо. В общем же случае свойство спуска Галуа отсутствует и $\Gamma(V)$ не допустимо.

ПримеР 1.2.12. Пусть $Y^{\prime}$ - гладкая проективная гиперэллиптическая кривая, рассматриваемая как двулистное накрытие проективной прямой $Y$. Тогда для $\mathscr{V}=\left(\Omega_{\mathscr{O} \mid k}^{1}\right)^{\otimes 2}$ тензорный квадрат любого регулярного дифференциала на $Y^{\prime}$ - Галуа-инвариантный элемент пространства $\Gamma\left(Y^{\prime}, \mathscr{V}_{Y^{\prime}}\right)$, который не лежит в подпространстве $\Gamma\left(Y, \mathscr{V}_{Y}\right)=0$.

Функтор $\Gamma$ совпадает с композицией забывающего функтора “общего слоя" в категорию $\mathscr{S} m_{G}(k)$ с функтором $\Gamma$, определенным после предложения 1.2.4. Функтор $\Gamma=\Gamma \circ \mathscr{S}$ - строгий на $\mathscr{A}$. Однако он не полон, и объекты в его образе очень сильно приводимы, см. пример 1.2 .5 (к которому можно добавить, что $\left.\mathscr{S}\left(\Omega_{F \mid k}^{1}\right)(Y)=\Gamma\left(Y, \Omega_{Y \mid k}^{1}\right)\right)$.

ГиПотезА 1.2.13. 1) Неприводимые обгекты $\mathscr{A}$ - прямые слагаемые обгек$m a \bigotimes_{F}^{\bullet} \Omega_{F \mid k}^{1}$.

2) Функторь $\operatorname{Hom}_{\mathscr{C}}\left(\bigotimes_{F}^{q} \mathfrak{m},-\right)$ точны на $\mathscr{A}$ для всех $q \geqslant 0$.

3) $\mathscr{A}$ эквивалентна категории "когерентных" пучков на $\mathfrak{S m}_{k}$.

Из справедливости гипотезы $1.2 .13,1)$ следовало бы, что естественное включение $\operatorname{Der}(k) \hookrightarrow \operatorname{Ext}_{\mathscr{A}}^{1}\left(\Omega_{F \mid k}^{1}, F\right)$, сопоставляющее ненулевому дифференцированию $\eta \in \operatorname{Hom}_{k}\left(\Omega_{k}^{1}, k\right)$ поля $k$ класс расширения $0 \rightarrow F \stackrel{\cdot \eta^{-1}(1)}{\longrightarrow} \Omega_{F}^{1} / \operatorname{ker} \eta \otimes_{k} F \rightarrow$ $\Omega_{F \mid k}^{1} \rightarrow 0$ в $\mathcal{A}$, является изоморфизмом. Это согласуется с [16; лемма 3.10$]$.

В качестве довода в пользу гипотезы $1.2 .13,2)$, в дополнение к случаю $k=\overline{\mathbb{Q}}$, в [16; теорема 2.4] показано, что для любого $L \subset F$, чисто трансцендентного степени $m$ над $k$, и любого $V \in \mathscr{A}$ любой неприводимый подфактор полулинейного представления $V^{G_{F \mid L}}$ группы $\mathrm{PGL}_{m+1} k$ над $L$ - прямое слагаемое алгебры $\bigotimes_{L}^{\bullet} \Omega_{L \mid k}^{1}$

Неприводимых гладких полулинейных представлений довольно много, и, в частности, большинство из них не допустимо. Например, никакой из факторов (в том числе неприводимых) циклического объекта $F\left[\left\{L \subset F \mid L \cong \bar{K}_{q}\right\}\right]^{\circ}$, состоящего из формальных $F$-линейных комбинаций степени нуль алгебраически замкнутых подполей в $F$ степени трансцендентности $q$ над $k$ для некоторого целого $q \geqslant 1$, не лежит в $\mathscr{A}$, см. [16; следствие 3.5]. Однако автор не знает даже, приводимы ли эти объекты. Тем более непонятно, как явно описать неприводимые объекты в $\mathscr{C}$. Так что, в части 2) гипотезы 1.2 .13 , категорию $\mathscr{A}$ нельзя заменить целой категорией $\mathscr{C}$, и нужно накладывать некоторые дополнительные условия, например, упомянутые в замечании 1.2.7, (ii). 
ЗАМЕЧАНИЕ 1.2.14. (i) Предполагая справедливой часть 2) гипотезы 1.2.13, можно переформулировать гипотезу 1.1.13 в следующей, лингвистически более убедительной, форме: любой неприводимый объект $\mathscr{A} d m\left(u \mathscr{I}_{G}\right)$ содержится в неприводимом объекте $\mathscr{A}$.

Эта переформулировка основана на предложении 4.1.17.

(ii) Далеко не для всякой абелевой категории имеет смысл изучать ее неприводимые объекты. Например, в факторкатегории категории векторных пространств над некоторым полем по ее подкатегории конечномерных векторных пространств неприводимых объектов нет вообще (равно как нет ни бесконечных сумм, ни бесконечных произведений).

С другой стороны, любой пучок векторных пространств или квазикогерентный пучок $\mathscr{F}$ на схеме $Y$ допускает фактор с носителем в одной точке $p$ :

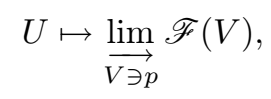

если $p \in U ; U \mapsto 0$, если $p \notin U$. Поэтому неприводимые объекты соответствуют точкам $Y$.

1.3. Обозначения, соглашения и терминология. Введем обозначения, которые будут использоваться на протяжении всей статьи.

Поля, их расширения, автоморфизмы и т. д. Пусть $K$ - поле и $H-$ некоторая группа его автоморфизмов. Мы считаем $H$ топологической группой, базу открытых подгрупп которой порождают стабилизаторы элементов $K$. Тогда $H$ становится хаусдорфовой и вполне несвязной группой, т. е. любая окрестность любого ее элемента содержит замкнутую подокрестность.

Для любого набора подмножеств $K_{0},\left(K_{\alpha}\right)_{\alpha \in I}$ поля $K$ подгруппа группы $H$, состоящая из элементов, неподвижных на $K_{0}$ и индуцирующих автоморфизмы каждого из $K_{\alpha}$, замкнута в $H$. В случае, когда $K_{\alpha}$ - подполя, естественный гомоморфизм из этой подгруппы в группу автоморфизмов поля $K_{\alpha}$ непрерывен. Через $G_{\left\{K,\left(K_{\alpha}\right)_{\alpha \in I}\right\} \mid K_{0}}$ мы обозначаем группу автоморфизмов поля $K$, действующих неподвижно на $K_{0}$ и сохраняющих все $K_{\alpha}$. Положим $G_{K \mid K_{0}}:=G_{\{K\} \mid K_{0}}$.

Если $K$ - подполе в $F$, то $\bar{K}$ обозначает алгебраическое замыкание $K$ в $F$, a $\operatorname{deg} \operatorname{tr}(F \mid K)$ - степень трансцендентности расширения $F \mid K$ (возможно бесконечную, но счетную).

Если явно не оговорено противное, то $F \mid k$ - расширение алгебраически замкнутых полей счетной (по умолчанию) или конечной степени трансцендентности $\operatorname{deg} \operatorname{tr}(F \mid k)=n \geqslant 1$ и $G:=G_{F \mid k}$. Везде, где не оговорено противное, характеристика $k$ - нулевая.

Общие обозначения. На протяжении всей работы $\mathbb{Q}$ - поле рациональных чисел и модуль - всегда $\mathbb{Q}$-векторное пространство (за исключением нескольких случаев, в которых характеристике поля $k$ разрешается быть положительной). Для абелевой группы $A$ положим $A_{\mathbb{Q}}=A \otimes \mathbb{Q}$.

Для многообразия $X$ над $k$ и любого расширения полей $E \mid k$ положим $X_{E}:=$ $X \times_{k} E . \mathbb{P}_{K}^{M}$ обозначает $M$-мерное проективное пространство над полем $K$.

Для коммутативной групповой схемы $A$ над $k$ положим $W_{A}=A(F) / A(k)$. 
Пусть $R$ - кольцо. Если $U$ - множество, то $R[U]$ обозначает свободный $R$-модуль с базисом $U$. Если $M-R$-модуль и $m \in M$, то $\langle m\rangle_{R}$ обозначает $R$-подмодуль в $M$, порожденный $m$. Если $R=\mathbb{Z}$, то $\langle m\rangle:=\langle m\rangle_{R}$. Через $\mathrm{Stab}_{w}$ обозначается стабилизатор элемента $w$.

Если $H$ - группа, действующая на множестве $S$ слева (например, $S$ - группа, а $H \subseteq S$ - ее подгруппа), то $H \backslash S$ обозначает множество $H$-орбит (правых смежных классов), а если $S_{1}$ - подмножество множества $S$, то $S \backslash S_{1}$ - дополнение в $S$ к $S_{1}$. Через $N_{H} H_{1}$ обозначаем нормализатор подгруппы $H_{1}$ в $H$.

Если $S$ - множество, то $|S|$ обозначает его мощность. Если $\alpha, \beta \in S$, то $\delta_{\alpha \beta}-$ символ Кронекера: $\delta_{\alpha \beta}=1$, если $\alpha=\beta$, и $\delta_{\alpha \beta}=0$, если $\alpha \neq \beta$.

Топологические группы, их представления, меры и т.д. Если $H-$ вполне несвязная топологическая группа, то через $H^{\circ}$ обозначим ее подгруппу, порожденную всеми компактными подгруппами, а через $H^{\text {ab }}$ - фактор $H$ по замыканию ее коммутанта. Очевидно, что $H^{\circ}-$ нормальная подгруппа в $H$, которая открыта, по крайней мере, если $H$ локально компактна.

$H$-множество (или представление группы $H$ в векторном пространстве над некоторым полем и т. п.) $W$ называется гладким, если стабилизаторы всех элементов $W$ открыты. Гладкое представление $W$ называется допустимым, если неподвижные векторы каждой открытой подгруппы образуют конечномерное подпространство в $W$.

$\mathbb{Q}\left(\chi_{H}\right)-H$-модуль правоинвариантных мер на $H$, и $\chi_{H}: H \rightarrow \mathbb{Q}_{+}^{\times}-$модуль, если $H$ локально компактна (см. текст после следствия 2.0.1). Положим $\mathbb{Q}(\chi):=\mathbb{Q}\left(\chi_{G}\right)$ и $\chi:=\chi_{G}$ при $n<\infty$.

$\mathscr{S} m_{H}(E)$ - категория гладких представлений группы $H$ над полем $E$ характеристики нуль. $\mathscr{A} d m_{H}(E)$ - ее полная подкатегория допустимых $E$-представлений. $\mathscr{I}_{G}(E)$ - полная подкатегория в $\mathscr{S} m_{G}(E)$, состоящая из таких представлений $W$ группы $G$, что $W^{G_{F \mid L}}=W^{G_{F \mid L^{\prime}}}$ для любого расширения $L \mid k$ в $F$ и любого чисто трансцендентного расширения $L^{\prime} \mid L$ в $F$. Когда речь идет о $\mathscr{I}_{G}(E)$, основным случаем будет $n=\infty$. Положим $\mathscr{S} m_{H}=\mathscr{S} m_{H}(\mathbb{Q})$ и $\mathscr{I}_{G}=\mathscr{I}_{G}(\mathbb{Q})$.

Алгебры Гекке $\mathscr{H}_{E}(H, U)$ для компактных подгрупп $U$ в $H$ определены в замечании 3.0.6, единица $\mathscr{H}_{E}(H, U)$ - мера Хаара на $U$ - обозначается $h_{U}$. Там же определено действие алгебры “мер" $\mathbb{D}_{E}(H):=\mathscr{H}_{E}(H,\{1\})$ на объектах $\mathscr{S} m_{H}(E)$. Положим $\mathbb{D}_{E}=\mathbb{D}_{E}(G)$ и $\mathscr{H}_{E}(U)=\mathscr{H}_{E}(G, U)$.

Фильтрация уровня $N$ • определена после теоремы 1.1.10.

\section{2. Структура $G$ и теории Галуа}

В этом разделе описаны основные результаты о топологической группе $G$ (в произвольной характеристике, см. теорему 2.2.1), такие как а) ее простота в случае $n=\infty$; б) простота ее (нормальной) подгруппы $G^{\circ}$, порожденной всеми компактными подгруппами. В частности, $G^{\circ}$ плотна в $G$ при $n=\infty$. Пункт а) дополняет результат Д. Ласкара [20]: $G$ проста как дискретная группа, если степень трансцендентности $F \mid k$ несчетна. 
Очевидно, что $G^{\circ}$ открыта при $n<\infty$, так что проекция $G \rightarrow G / G^{\circ}$ с дискретной топологией на $G / G^{\circ}$ непрерывна. При $n=1$ лемма 2.4.7 представляет $G / G^{\circ}$ как фактор некоторой, довольно "структурированной", группы. О группе $G / G^{\circ}$ известно немного, отчего обычно приходится работать в "стабильном" случае $n=\infty$ и ставить вопросы так, чтобы можно было обойтись без знания структуры этой группы.

Если $n=\infty$, то из простоты группы $G$ следует (см. [7; следствие 2.11]), что любой нетривиальный непрерывный гомоморфизм из $G$ инъективен; а в случае $n<\infty$ любой неинъективный непрерывный гомоморфизм из $G$ пропускается через $G / G^{\circ}$. Еще одно следствие состоит в том, что у группы $G^{\circ}$ (и у $G$, если $n=\infty)$ нет ни нетривиальных гладких представлений конечной степени, ни собственных замкнутых подгрупп конечного индекса.

СлЕДСтвиЕ 2.0.1 [7; следствие 2.11]. Для любой подгруппы $H$ группы $G$, содержащей $G^{\circ}$, и любого непрерьвного гомоморфизма $\pi$ из $H$ или $\pi$ ингективен, или ограничение $\pi$ на $G^{\circ}$ тривиально.

Пусть $H$ - локально компактная группа и $\mathbb{Q}\left(\chi_{H}\right)$ - фактор свободной абелевой группы, порожденной множеством компактных открытых подгрупп в $H$, по соотношениям $[U]=\left[U: U^{\prime}\right] \cdot\left[U^{\prime}\right]$ для всех $U^{\prime} \subset U$. Поскольку пересечение любой пары компактных открытых подгрупп в $H$ имеет конечный индекс в каждой из них, $\mathbb{Q}\left(\chi_{H}\right)$ - одномерное $\mathbb{Q}$-векторное пространство, ориентированное условием $[U]>0$ для любой $U$. Другими словами, $\mathbb{Q}\left(\chi_{H}\right)$ - пространство $\mathbb{Q}$-значных правоинвариантных мер на $H$. На этом пространстве действует группа бинепрерывных автоморфизмов группы $H$. В частности, группа $H$ действует на нем сопряжением. Пусть $\chi_{H}: H \rightarrow \mathbb{Q}_{+}^{\times}-$характер этого представления $H$, называемый модулем группы $H$. Он тривиален в ограничении на (открытую) подгруппу $H^{\circ}$, порожденную всеми компактными подгруппами $H$.

В п. 2.5 вводится локально компактная группа $\mathfrak{G}$. Если $n<\infty$, то $\mathfrak{G}:=G$, а если $n=\infty$, то имеется непрерывное вложение $\mathfrak{G} \hookrightarrow G$ с плотным образом. Из явного описания модуля $\chi=\chi_{\mathfrak{G}}$ видно, что $\chi$ сюръективен при всех $1 \leqslant$ $n \leqslant \infty$. Из теоремы 2.2.1 вытекает, что группа $\mathfrak{G}^{\circ}$ топологически проста.

Однако автор не знает даже, тривиальна ли дискретная группа $\operatorname{ker} \chi / \mathfrak{G}^{\circ}$. Если она тривиальна при $n=1$, то она тривиальна и в общем случае (см. [7; лемма 2.15]).

2.1. Теория Галуа для компактных подгрупп. Пусть $F \mid k$ - произвольное расширение произвольных полей и $G=G_{F \mid k}-$ его группа автоморфизмов. Топология на $G$, описанная во введении, с. 88 , изучалась в работах $[1$; с. 151 , упражнение 5$],[2],[3 ;$ гл. $6, \S 6.3]$ и [4; гл. 2 , часть $1, \S 1]$. Там показано, что $G$ хаусдорфова и вполне несвязна и что для любого подполя $K$ в $F \mid k$ топология на $G_{F \mid K}$ совпадает с ограничением топологии на $G$. Подгруппы $G_{\left\{F,\left(F_{\alpha}\right)_{\alpha \in I}\right\} \mid k}$ замкнуты в $G$, поскольку если $\sigma\left(F_{\alpha}\right) \subseteq F_{\alpha}, \sigma\left(F \backslash F_{\alpha}\right) \subseteq F \backslash F_{\alpha}$ и $\sigma(F)=F$, то $\sigma\left(F_{\alpha}\right)=F_{\alpha}$.

Теория Галуа-Крулля сопоставляет промежуточные подполя некоторого расширения Галуа замкнутым (или, что то же, компактным) подгруппам группы Галуа этого расширения, и обратно. А именно, подгруппе сопоставляется 
ее поле инвариантов, а подполю сопоставляется группа автоморфизмов, неподвижных на нем.

Эти сопоставления взаимно обратны, и допускают следующее прямое обобщение.

ОПРедЕЛЕНИЕ 2.1.1. (а) Непустой набор $\mathscr{L}$ подполей в $F$ называется $p$ шетом, если с любым своим элементом он содержит и любое его расширение. Назовем насыщением произвольного набора $\mathscr{L}$ подполей в $F$ решето $\overline{\mathscr{L}}$, элементы которого - это расширения в $F$ элементов $\mathscr{L}$.

(b) Набор подполей называется направленным, если вместе с любой парой своих элементов он содержит и их пересечение.

ПримеР 2.1.2. (i) Насыщение наборов, состоящих из единственного подполя, определяет вложение множества подполей в $F \mid k$ во множество решет подполей в $F \mid k$.

(ii) Для убывающей последовательности $K_{1} \supseteq K_{2} \supseteq K_{3} \supseteq \cdots$ подполей $K_{j}$ в $F \mid k$, над которыми поле $F$ алгебраично, набор всех расширений всех $K_{j}-$ направленное решето. Ясно, что насыщения наборов $\left(K_{j}\right)_{j \geqslant 1}$ и $\left(K_{j}\right)_{j \in S}$ совпадают для любого бесконечного подмножества $S$ натуральных чисел.

ПреДЛОЖЕНИЕ 2.1.3. Формула $K \mapsto \operatorname{Aut}(F \mid K)=: G_{F \mid K}$ задает морфизм

$$
\beta:\{\text { подполя в } F \text { над } k\} \rightarrow\{\text { замкнутые подгруппы в } G\}
$$

унитарных полугрупп (который преобразует композит полей в пересечение подгрупп), обращающий включения, инбективный, если $\operatorname{ch} \operatorname{ar}(k)=0$ и $F$ алгебраччески замкнуто. Он сохраняет единицы: $k \mapsto G$. Образ $\beta$ стабилен относительно переходов $к$ подгруппам, факторы по которым компактны, и к бо́льшим подгруппам с компактными факторами; если F алгебраччески замкнуто, то слои $\beta$ состоят из подполей поля $F$ с общим совершенным замыканием; $\beta$ индуцирует согласованные биекции

$$
\left\{\begin{array}{c}
\text { направленные решета, } \\
\text { состоящие из таких } K \text { в } F \mid k, \\
\text { что } F \mid K-\text { расширения Галуа }
\end{array}\right\} \stackrel{A}{\longleftrightarrow}\left\{\begin{array}{c}
\text { подгруппы } G, \text { исчерпываемые } \\
\text { компактными подгруппами }
\end{array}\right\}
$$

$\left\{\begin{array}{c}\text { такие подполя } K \text { в } F \mid k, \text { что } \\ F \mid K-\text { расширение Галуа }\end{array}\right\} \quad \stackrel{B}{\longleftrightarrow}\{$ компактные подгруппы в $G\}$

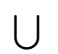

$$
\left\{\begin{array}{c}
\text { такие подполя } K \text { в } F \mid k, \text { что } \\
\text { существует такое подполе } L \\
\text { в } K \mid k \text { конечного типа, что } F \mid K^{\prime}- \\
\text { расширение Галуа только для } \\
K^{\prime}=K, \text { если } K^{\prime}-\text { подполе в } K \mid L
\end{array}\right\} \stackrel{C}{\longleftrightarrow} \quad\left\{\begin{array}{c}
\text { компактные открытые } \\
\text { подгруппы в } G
\end{array}\right\} \text {. }
$$

Обратные отображения в случаях $B$ и $C$ заданы формулой $G \supset H \mapsto F^{H}$ (неподвижное подполе в $F$ группъ $H$ ). 
Вполне несвязная топологическая группа локально компактна тогда и только тогда, когда в ней имеется открытая компактная подгруппа. В частности, если $\operatorname{deg} \operatorname{tr}\left(F \mid F^{G}\right)<\infty$ (например, если $F=\bar{F}$ и $\left.n<\infty\right)$, то $G$ локально компактна.

Соответствие $B$ установлено в $[2 ; \S 3$, лемма 1] (см. также [3; предложение 6.11]); по поводу соотношения $C$ см. [1], оно также непосредственно следует из [2; $\S 3$, лемма 1$]$ и приведено в [3; предложение 6.12$]$. В случае алгебраически замкнутого $F$ в левой части соответствия $C$ стоят такие совершенные замыкания $K$ в $F$ подполей конечного типа над $k$, что $F \mid K$ - расширение Галуа. Соответствие $A$ индуцируется отображениями

$$
\left\{\begin{array}{c}
\text { подгруппы в } G, \text { порожденные } \\
\text { компактными подгруппами }
\end{array}\right\} \leftrightarrows^{\gamma}\left\{\begin{array}{c}
\text { решета, состоящие } \\
\text { из таких подполей в } F \mid k, \\
\text { над которыми } F \text { алгебраично }
\end{array}\right\}
$$

заданными формулами $\beta: G \supset H \mapsto\left(F^{C} \subseteq F\right)_{C}$, где $C$ пробегает компактные подгруппы в $H$, и $\gamma: \mathscr{L} \mapsto \bigcup_{K \in \mathscr{L}} G_{F \mid K}$.

Примеры 2.1.4. (i) Если $F$ алгебраически замкнуто, то подполя в $F \mid k$, над которыми $F$ - расширение Галуа, - это совершенные подполя, содержащие $k$, над которыми $F$ алгебраично. Если, кроме того, $n=1$, то собственные подгруппы в образе $\beta$ - это компактные подгруппы.

(ii) Пусть $\operatorname{char}(k)=0, F=\overline{k(x)}$ и группа $H$ порождена компактными подгруппами $G_{F \mid k\left((x-a)^{2}\right)}$ для $a \in k$. Зафиксируем вложение $F \hookrightarrow k\left(\left(x^{-1 / \infty}\right)\right)$ и определим очевидным образом действие сдвигов на элементы $k$ на формальных рядах Пюизё. Это задаст вложение групп $k \hookrightarrow G$. Пусть $\Gamma$ - образ $k \hookrightarrow G$. Тогда умножение $G_{F \mid k\left(x^{2}\right)} \times \Gamma \rightarrow H$ биективно. Ясно, что пересечение $H$ с объединением компактных подгрупп $G$ группой не является.

(iii) Группа $G$ может быть локально компактной, даже если степень трансцендентности $F \mid F^{G}$ бесконечна. Например, пусть многочлен $P(X, Y, Z)$ над $k$ определяет поверхность без бирациональных автоморфизмов, $x, y_{i}$, где $i \in \mathbb{Z}$, независимые переменные и $z_{i}$ удовлетворяют условиям $P\left(\tau^{i} x, y_{i}, z_{i}\right)=0$ для всех $i \in \mathbb{Z}$ и некоторого элемента $\tau \in G_{k(x) \mid k}$ бесконечного порядка (например, $\tau x=x+1$, если $\operatorname{char}(k)=0$, или $\tau x=q x$, если $\left.q \in k^{\times} \backslash \mu_{\infty}\right)$. Положим $F=k\left(x, y_{i}, z_{i} \mid i \in \mathbb{Z}\right)$. Тогда $G_{F \mid k(x)}=\{1\}$, т. е. группа дискретна. С другой стороны, $G$ содержит такой элемент $\sigma$, что $\sigma x=\tau x, \sigma y_{i}=y_{i+1}$ и $\sigma z_{i}=z_{i+1}$ для всех $i \in \mathbb{Z}$, и, значит, $F^{G}=F^{\langle\sigma\rangle}=k$.

(iv) Отображение $H \mapsto F^{H}$ обращает порядок, но оно не согласовано, вообще говоря, с $\beta$. Если $F$ алгебраически замкнуто, то оно обратно слева к ограничению $\beta$ на совершенные подполя, любое совершенное подполе имеет вид $F^{H}$ и $G$ содержит в качестве подфакторов группы автоморфизмов всех расширений в $F \mid k$. Но $H \mapsto F^{H}$ не является морфизмом моноидов: группы $G_{\left\{F, k\left(x^{2}\right)\right\} \mid k}, G_{\left\{F, k\left((x+1)^{2}\right)\right\} \mid k}$ отображаются в $k$, в то время как $G_{\left\{F, k\left(x^{2}\right)\right\} \mid k} \cap$ $G_{\left\{F, k\left((x+1)^{2}\right)\right\} \mid k}=G_{F \mid k(x)}$ переходит в $k(x) \neq k$.

Дополнительные примеры можно найти в [21]. 
2.2. Топологическая простота $G^{\circ}$ и $G$. Отныне поле $F$ будет алгебраически замкнутым.

Скажем, что топологическая группа топологически проста, если любая ее замкнутая нормальная собственная подгруппа тривиальна.

Теорема 2.2.1 [7; теорема 2.9]. Если $n<\infty$, то любая нетривиальная подгруппа в $G$, нормализуемая подгруппой $G^{\circ}$, плотна в $G^{\circ}$. Eсли $n=\infty$, то любая нетривиальная нормальная подгруппа в $G$, например $G^{\circ}$, плотна.

Здесь $F \mid k$ - произвольное расширение алгебраически замкнутых полей произвольной характеристики. В частности, подгруппа $G^{\circ}$ группы $G$ топологически проста, если $n<\infty$; если $n=\infty$, то $G$ топологически проста.

ЗАмечАниЕ 2.2.2. (i) Если $n=1$ и $\operatorname{char}(k) \neq 0$, то сепарабельное замыкание подполя $k(x)$ в $F$ порождено $G^{\circ}$-орбитой $x$ для любого $x \in F \backslash k$, см. предложение 3.3.1.

(ii) Рассуждение в работе [20] показывает, что $G$ проста как дискретная группа, если степень трансцендентности $F$ над $k$ несчетна.

То, что $G^{\circ}$ плотна в $G$ при $n=\infty$, вытекает из следующей леммы.

Лемма 2.2.3. Пусть $L$ - подполе поля $F$ такое, что $\operatorname{deg} \operatorname{tr}(F \mid L)=\infty$. Тогда $G_{F \mid L}$ - замыкание множества произведений пар элементов компактных noдгрynn в $G_{F \mid L}$.

ДоказАТЕЛЬство. Пусть $\sigma \in G_{F \mid L}$. Надо показать, что ограничение $\sigma$ на любое конечное подмножество $S \subset F$ совпадает с ограничением произведения пары элементов компактных подгрупп $G_{F \mid L}$. Пусть $T \subset F$ - подмножество порядка $|S|$, элементы которого алгебраически независимы над $L(S, \sigma(S))$. Выберем в алгебраическом замыкании $L(T)$ подполе $K$, изоморфное над $L$ полю $L(S)$. Ясно, что найдутся такие элементы компактных подгрупп $\tau_{1}, \tau_{2}$, что $\tau_{1}$ переставляет $L(S)$ и $K, \tau_{2}$ переставляет $L(\sigma(S))$ и $K$ и $\left.\tau_{2} \tau_{1}\right|_{S}=\left.\sigma\right|_{S}$.

ПРЕДЛОЖЕНИЕ 2.2.4 [7; предложение 2.14], [19]. Пусть $L_{1} u L_{2}-$ подполя в $F$ такие, что $\bar{L}_{1} \cap \bar{L}_{2}$ алгебраично над $L_{1} \cap L_{2}$ и либо $\operatorname{deg} \operatorname{tr}\left(F \mid L_{2}\right)=\infty$, либо $\operatorname{deg} \operatorname{tr}\left(L_{1} \mid L_{1} \cap L_{2}\right)<\infty$ и $L_{1}$ и $L_{2}$ алгебраически замкнуты. Тогда подгруппа в $G$, порожденная $G_{F \mid L_{1}} u G_{F \mid L_{2}}$, плотна в $G_{F \mid L_{1} \cap L_{2}}$.

ЗАмЕчАНИЕ 2.2.5. Это предложение (и [7; лемма 2.16], см. доказательство приведенной ниже леммы 2.2.6) - аналог результата работы [22]: алгебра Ли дифференцирований $\operatorname{Der}\left(F \mid L_{1} \cap L_{2}\right)$ топологически порождена подалгебрами Ли $\operatorname{Der}\left(F \mid L_{1}\right)$ и $\operatorname{Der}\left(F \mid L_{2}\right)$. (Базу открытых подалгебр Ли в $\operatorname{Der}(F \mid L)$ задают аннуляторы конечных подмножеств в $F$.)

Лемма 2.2.6. Пусть $L_{0} \subset L_{1} \subset L_{2}$ - пара нетривиалъных чисто трансцендентных расширений в F конечного типа. Пусть $\mathfrak{S}$ - некоторая транзитивная группа перестановок базиса трансцендентности $S$ поля $L_{2}$ над $L_{0}$, продолжающего базис трансцендентности поля $L_{1}$ над $L_{0}$. Пусть $H$ - подгруппа в $G_{F \mid L_{0}}$, сохраняющая $L_{2}$ и проектирующаяся на подгруппу в $G_{L_{2} \mid L_{0}}$, содержащую S. Тогда подгруппа $G^{\prime}$ в $G$, порожденная $G_{F \mid L_{1}} u H$, совпадает $c G_{F \mid L_{0}}$. 
ДокАЗАтельство. Группа $G^{\prime}$ содержит подгруппы, сопряженные с $G_{F \mid L_{1}}$

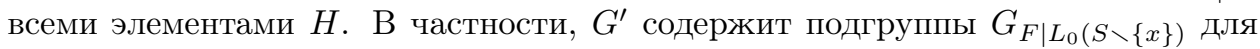
всех $x \in S$. Согласно [7; лемма 2.16], группа, порожденная подгруппами $G_{F \mid L(S \backslash\{x\})}$ для всех $x \in S$, плотна в $G_{F \mid L}$ для любого подполя $L$ в $F$ и некоторого множества $S$ элементов $F$, алгебраически независимых над $L$. Поэтому $G^{\prime}$ совпадает с $G_{F \mid L_{0}}$.

\section{3. Открытые и максимальные собственные подгруппы; теории} Галуа. (Большую часть результатов этого пункта можно найти в [19].) Изучение гладких представлений $G$ и стабилизаторов их векторов приводит к изучению открытых подгрупп $G$. Для любого $\operatorname{deg} \operatorname{tr}(F \mid k)=n \leqslant \infty$ имеется морфизм коммутативных ассоциативных моноидов с (минимальной) единицей, обращающий включения:

$$
\alpha:\left\{\begin{array}{c}
\text { открытые } \\
\text { подгруппы } G
\end{array}\right\} \rightarrow\left\{\begin{array}{c}
\text { алгебраически замкнутые } \\
\text { подполя поля } F \text { конечной } \\
\text { степени трансцендентности над } k
\end{array}\right\}=: A \Pi \text {. }
$$

Морфизм $\alpha$ преобразует пересечение подгрупп в алгебраическое замыкание композита подполей, а единицу $G$ - в единицу $k$. Он однозначно определяется следующими эквивалентными условиями:

- каждая открытая подгруппа $U \subseteq G$ содержит $G_{F \mid \alpha(U)}$ в качестве нормальной подгруппы ${ }^{2}$ и, по возможности, $\alpha(U) \neq F$;

- $G_{F \mid \alpha(U)} \subseteq H$ и степень трансцендентности $\alpha(U)$ над $k$ минимальна.

В частности, для любого нетривиального алгебраически замкнутого расширения $L \neq F$ поля $k$ конечной степени трансцендентности в $F$ нормализатор $G_{\{F, L\} \mid k}$ в $G$ подгруппы $G_{F \mid L}$ (который, очевидно, открыт) максимален среди собственных подгрупп $G$.

При $n=\infty$ любая собственная открытая подгруппа $G$ содержится в максимальной собственной открытой подгруппе $G$ и любая максимальная собственная открытая подгруппа $G$ имеет вид $G_{\{F, L\} \mid k}$ для некоторого $L \in A \Pi, L \neq k$.

Вопросы. 1. Прообраз любой подгруппы простого (конечного) индекса в $\mathbb{Q}_{+}^{\times}$относительно характера модуля при $n<\infty$ - это еще один тип максимальных открытых собственных подгрупп. Любое компактное подмножество группы $G$ содержится в бесконечном множестве таких подгрупп. Существуют ли другие максимальные открытые собственные подгруппы?

2. Существуют ли замкнутые подгруппы, не содержащиеся в максимальных?

3. Реализуются ли максимальные собственные открытые подгруппы в качестве стабилизаторов неприводимых (полу-) линейных представлениях (при $n=\infty$ ) группы $G$ ? (Ответ был бы отрицательным, если бы представления $\mathbb{Q}[\{[\bar{L}] \mid k \subset L \subset F, \operatorname{deg} \operatorname{tr}(L \mid k)=m\}]^{\circ}[$ соответственно $F[\{[\bar{L}] \mid k \subset L \subset F$, $\operatorname{deg} \operatorname{tr}(L \mid k)=m\}]^{\circ} \in \mathscr{C}$ ] оказались неприводимыми для всех $m \geqslant 1$.)

В случае произвольной степени трансцендентности стабилизаторы дискретных нормирований ранга один - это еще один тип замкнутых максимальных,

\footnotetext{
${ }^{2}$ т. е. $U$ содержится в нормализаторе $G_{\{F, \alpha(U)\} \mid k}$ подгруппы $G_{F \mid \alpha(U)}$.
} 
но уже не открытых подгрупп, см. предложение 2.4.5. С их помощью по представлениям $G$ строятся функторы на категориях гладких $k$-многообразий, см. формулу (1), предложение 4.4.12 и пояснительные тексты к ним.

ЗАМЕЧАНИЕ 2.3.1. (i) Если $n<\infty$ и $H \subset G$ не содержится ни в одной из подгрупп вида $G_{\{F, L\} \mid k}$ ни для какого $L \in A \Pi \backslash\{k, F\}$, то $F$ алгебраично над подполем, порожденным над $k H$-орбитой $x$ для любого $x \in F \backslash k$.

(ii) Если $\operatorname{deg} \operatorname{tr}(L \mid k)=\operatorname{deg} \operatorname{tr}(F \mid L)=\infty$, то $G_{\{F, \bar{L}\} \mid k}$ максимальна среди замкнутых собственных подгрупп $G$, т. е. подгруппа $H$, порожденная $G_{\{F, \bar{L}\} \mid k}$ и любым $\sigma \in G$ таким, что $\sigma(\bar{L}) \neq \bar{L}$, плотна в $G$. Вопрос: нельзя ли условие "degtr $(F \mid L)=\infty$ " заменить условием " $F \neq \bar{L} "$ ?

(iii) Пусть подполя $\bar{K}$ и $\bar{L}$ находятся в общем положении. Тогда имеется ровно три максимальных собственных открытых подгруппы группы $G$, содержащих $G_{\{F, \bar{K}, \bar{L}\} \mid k}$, а именно, $G_{\{F, \bar{K}\} \mid k}, G_{\{F, \bar{L}\} \mid k}$ и $G_{\{F, \overline{K L}\} \mid k}$, поскольку если $\sigma \in G$ не сохраняет $\bar{K}, \bar{L}$ и $\overline{K L}$, то $\left\langle\sigma, G_{\{F, \bar{K}, \bar{L}\} \mid k}\right\rangle=G$.

Объединение собственных открытых подгрупп $G$ характеризуется следующим фактом.

СЛЕДСТВИЕ 2.3.2 [19]. При $n=\infty$ оббединение собственных открытых nодгрупп группъ $G$ плотно в $G$ и не совпадает с $G$. Следующие свойства элемента $\sigma \in G$ эквивалентны:

1) $\sigma$ не лежит в обгединении собственных открытых подгрупп $G$;

2) $W^{\langle\sigma\rangle}=W^{G}$ для любого гладкого $G$-множества $W$;

3) любое $\sigma$-инвариантное конечномерное $F$-векторное подпространство в $\Omega_{F \mid k}^{1}-$ нулевое.

ЗАмЕчАНИЕ 2.3.3. При $n=\infty$ любая счетная свободная группа $H=*_{j \in S} \mathbb{Z}$ вкладывается в $G$ так, что ее пересечение с любой собственной открытой подгруппой в $G$ тривиально. А именно, выберем базис трансцендентности $F \mid k$ и пронумеруем его элементами группы $H:\left\{x_{h} \mid h \in H\right\}$. Определим действие образующих $\left\{h_{j} \mid j \in S\right\}$ группы $H$ на базисе трансцендентности формулой $h_{j} x_{h}=x_{h_{j}}$. Ясно, что это действие продолжается, хотя и неоднозначно, на $F$.

Пусть $n=\infty, \sigma$ не лежит в объединении собственных открытых подгрупп $G$ и $A=A_{\sigma}:=F\left\langle\sigma, \sigma^{-1}\right\rangle$ - алгебра эндоморфизмов аддитивной группы $F$, порожденная $F$ и $\sigma^{ \pm 1}$. Ясно, что $A$ - евклидово простое кольцо с центром $F^{\langle\sigma\rangle}$ (см. [23]). Стандартным образом проверяется, что $A$-модули без кручения свободны. В частности, $\Omega_{F \mid k}^{1}$ является свободным $A$-модулем не более чем счетного ранга. Ранг $\Omega_{F \mid k}^{1}$ как $A$-модуля - инвариант класса сопряженности $\sigma$. Других инвариантов автору не известно.

Еще одна теория Галуа, [19]. В. Крулль в [24] задал вопрос, как построить теорию Галуа трансцендентных расширений $F \mid k$, т. е. как построить подгруппы $H$, совпадающие с группами автоморфизмов $F$ над неподвижными подполями $F^{H}$ ? Для алгебраически замкнутых полей счетной степени трансцендентности такая, хотя и не очень явная, теория получается как следствие описания максимальных открытых подгрупп группы $G$. 
А именно, можно охарактеризовать

1) нормализаторы $G_{\{F, L\} \mid k}$ подгрупп $G_{F \mid L}$ в $G$ для всех $L \in A \Pi \backslash\{k\}$ как максимальные открытые собственные подгруппы $G$;

2) подгруппы $G_{F \mid L}$ для всех $L \in A \Pi \backslash\{k\}$ как минимальные замкнутые нетривиальные нормальные подгруппы в $G_{\{F, L\} \mid k}$ (это следует из топологической простоты $G)$;

3) подгруппы $G_{F \mid L}$ группы $G$ для всех нетривиальных расширений $L \mid k$ в $F$ конечного типа как открытые подгруппы, содержащие нормальные кокомпактные подгруппы вида $G_{F \mid \bar{L}}$ из п. 2) (это следует из классической теории Галуа для $\bar{L} \mid k)$;

4) собственные подгруппы в образе $\beta$ как пересечения подгрупп из п. 3).

ЗАмечАние 2.3.4. Подгруппы $G_{F \mid L}$ группы $G$ для всех расширений $L \mid k$ в $F$ конечного типа и степени трансцендентности один - это подгруппы из п. 3), которые содержатся в единственной максимальной собственной подгруппе группы $G$.

2.4. Нормирования и связанные с ними подгруппы. (Основные результаты этого пункта содержатся в [19].) Пусть $\mathscr{O}_{v}-$ кольцо нормирования в $F, \mathfrak{m}_{v}=\mathscr{O}_{v} \backslash \mathscr{O}_{v}^{\times}-$максимальный идеал и $\kappa(v)-$ поле вычетов $v$. Если $k \subseteq \mathscr{O}_{v}$, то зафиксируем подполе $k \subseteq F^{\prime} \subseteq \mathscr{O}_{v}$, отождествляемое редукцией по модулю $\mathfrak{m}_{v}$ с $\kappa(v)$. В этом случае поле вычетов $\kappa(v)$ алгебраически замкнуто и его характеристика равна нулю.

Положим $G_{v}:=\left\{\sigma \in G \mid \sigma\left(\mathscr{O}_{v}\right)=\mathscr{O}_{v}\right\}$. Подгруппа $G_{v}$ замкнута в $G$.

Группа нормирования $\Gamma:=F^{\times} / \mathscr{O}_{v}^{\times} \cong \mathbb{Q}^{r}$ вполне упорядочена: $v(x) \geqslant v(y)$ тогда и только тогда, когда $x y^{-1} \in \mathscr{O}_{v}$, где $v: F^{\times} \rightarrow \Gamma$ - естественная проекция.

Назовем рангом $v$ число $r=\operatorname{dim}_{\mathbb{Q}} \Gamma$. Будем всегда считать его конечным.

Допустим, что характеристики поля $L$ и поля вычетов $\kappa$ некоторого его нормирования $w$ совпадают. Тогда $w$ называется дискретным, если $L$ алгебраично над подполем, порожденным некоторым подъемом базиса трансцендентности $\kappa$ и некоторым подъемом базиса группы значений нормирования. В частности, $v$ дискретно тогда и только тогда, когда степень трансцендентности $F$ над $F^{\prime}$ равна $r$.

Выберем произвольное алгебраически замкнутое подполе $F^{\prime}$ в $F$, над которым степень трансцендентности $F$ равна $r$, базис трансцендентности $x_{1}, \ldots, x_{r}$ расширения $F \mid F^{\prime}$ и вложения $F \hookrightarrow \lim _{N} F^{\prime}\left(\left(x_{1}^{1 / N}\right)\right) \ldots\left(\left(x_{r}^{1 / N}\right)\right)$ над $F^{\prime}\left(x_{1}, \ldots, x_{r}\right)$. В этом случае $v\left(x_{1}^{m_{1}}\right)<\cdots<v\left(x_{r}^{m_{r}}\right)$ для всех $m_{1}, \ldots, m_{r}>0$.

Если $r<\infty$ и $\sigma\left(\mathscr{O}_{v}\right) \subseteq \mathscr{O}_{v}$ для некоторого $\sigma \in G$, то $\sigma \in G_{v}$, так как $\sigma$ индуцирует сюръективный эндоморфизм $\Gamma$, т. е. автоморфизм.

Как известно (см. [25] или [26; гл. 5, упражнение 32]), для любого кольца нормирования $\mathscr{O}_{p}$ с группой нормирования Г отображение $\mathfrak{p} \mapsto\left\langle v\left(\mathscr{O}_{p} \backslash \mathfrak{p}\right)\right\rangle$ задает биекцию между простыми идеалами $\mathscr{O}_{p}$ и изолированными подгруппами в $\Gamma$, причем $v\left(\mathscr{O}_{p} \backslash \mathfrak{p}\right)$ - множество всех неотрицательных элементов соответствующей изолированной подгруппы Г. Поэтому в $\mathscr{O}_{v}$ имеется ровно $r+1$ простой идеал. 
ЗАмЕчАниЕ 2.4.1. (i) Если $\mathfrak{p} \neq 0$ - не максимальный простой идеал конечной коразмерности в $\mathscr{O}_{v}$ и $\mathscr{O}_{v^{\prime}}:=\left(\mathscr{O}_{v}\right)_{\mathfrak{p}}$, то $G_{v} \subseteq G_{v^{\prime}}$ (так как любой элемент $\sigma \in G_{v}$ сохраняет $\mathfrak{p}$, а значит, и $\mathscr{O}_{v} \backslash \mathfrak{p}$, т. е. индуцирует автоморфизм $\left.\mathscr{O}_{v^{\prime}}\right)$.

(ii) При $r>1$ имеется собственное включение $G_{v} \subset G_{\left\{F, \mathscr{O}_{v}\left[x_{1}^{-1}\right]\right\} \mid k}$, т. е. $G_{v}$ не максимальна.

Пусть $\mathscr{P}_{L}^{r}-$ множество колец дискретного нормирования ранга $r$ в $L$, содержащих $k$. Множество $\mathscr{P}_{L}^{r}$ допускает следующее описание.

Пусть $\mathscr{C}_{X}^{r}-$ множество цепочек до коразмерности $r$ неприводимых нормальных подмногообразий на полном неприводимом нормальном многообразии $X$ над $k$. Любая собственная сюръекция с неприводимыми слоями (например, бирациональный морфизм) $X^{\prime} \stackrel{\pi}{\longrightarrow} X$ задает вложение $\mathscr{C}_{X}^{r} \hookrightarrow \mathscr{C}_{X^{\prime}}^{r}$ по формуле $\left(Z^{1} \supset \cdots \supset Z^{r}\right) \mapsto\left(W^{1} \supset \cdots \supset W^{r}\right)$, где $W^{0}:=X^{\prime}$ и $W^{j}-$ собственный прообраз $Z^{j}$ относительно ограничения $\pi$ на $W^{j-1}$ для $1 \leqslant j \leqslant r$. Если $L$ конечно порождено над $k$, то $\mathscr{P}_{L}^{r} \cong \lim _{X} \mathscr{C}_{X}^{r}$, где $X$ пробегает модели $L \mid k$,

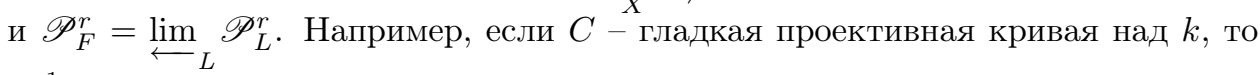
$\mathscr{P}_{k(C)}^{1}-$ множество замкнутых схемных точек $C$.

Любая собственная сюръекция $X^{\prime} \stackrel{\pi}{\rightarrow} X$ задает вложения $\mathbb{Z}\left[\mathscr{C}_{X}^{r}\right] \hookrightarrow \mathbb{Z}\left[\mathscr{C}_{X^{\prime}}^{r}\right]$ и $\mathbb{Z}\left[\mathscr{C}_{X}^{r}\right]^{\circ} \hookrightarrow \mathbb{Z}\left[\mathscr{C}_{X^{\prime}}^{r}\right]^{\circ}$, где $\mathbb{Z}\left[\mathscr{C}_{X}^{r}\right]^{\circ}:=\bigcap_{j=0}^{r-1} \operatorname{ker}\left(\mathbb{Z}\left[\mathscr{C}_{X}^{r}\right] \rightarrow \mathbb{Z}\left[\mathscr{C}_{X}^{r, j}\right]\right), \mathscr{C}_{X}^{r, j}$ обозначает множество цепочек без компоненты коразмерности $j$ и $\mathscr{C}_{X}^{r} \rightarrow \mathscr{C}_{X}^{r, j}-$ выбрасывание такой компоненты.

Пусть $\bigsqcup^{r}:=\lim _{L} \mathbb{Z}\left[\mathscr{P}_{L}^{r}\right]^{\circ}$, где $L \mid k$ пробегает расширения конечного типа в $F$. Тогда формула

$$
\begin{aligned}
\operatorname{Res}\left(\alpha \frac{d t_{1}}{t_{1}} \wedge \cdots \wedge \frac{d t_{q}}{t_{q}}\right):= & \left.\sum_{\sigma \in \mathfrak{S}_{q}} \operatorname{sgn}(\sigma) \alpha\right|_{D_{1 \ldots q}} \\
& \times\left(D_{\sigma(1)} \supset D_{\sigma(1) \sigma(2)} \supset \cdots \supset \bigcap_{j=1}^{q} D_{j}=: D_{1 \ldots q}\right),
\end{aligned}
$$

где $D_{i}$ задан локально уравнением $t_{i}=0$ и $\alpha$ регулярна в некоторой окрестности $t_{1}=\cdots=t_{q}=0$, определяет морфизм гладких $G$-модулей $\operatorname{gr}_{2 q}^{W} H_{\mathrm{dR} / k}^{q}(F) \stackrel{\text { Res }}{\longrightarrow}$ $Ц^{q} \otimes k$.

Лемма 2.4.2. Если $0 \leqslant r<n+1 \leqslant \infty$, то $G$ действует транзитивно на множестве пар $(v, \Lambda)$, где $v: F^{\times} \rightarrow \Gamma \cong \mathbb{Q}^{r}$ представляет элемент $\mathscr{P}_{F}^{r}$ и $\Lambda \cong \mathbb{Z}^{r}$ - решетка в Г. Стабилизатор парь $(v, \Lambda)$ действует транзитивно на множестве максимальных подполей $\widetilde{F}$ в $F \mid k$ таких, что $v\left(\widetilde{F}^{\times}\right)=\Lambda$. Поле вычетов поля $\widetilde{F}$ совпадает с $\kappa(v)$ (в частности, оно алгебраически замкнуто).

Действие $G_{v}$ на $\kappa(v)$ задает гомоморфизм $G_{v} \rightarrow G_{\kappa(v) \mid k}$. Определим подгруппу "инерции" как его ядро: $G_{v}^{\dagger}:=\left\{\sigma \in G_{v} \mid \sigma x-x \in \mathfrak{m}_{v}\right.$ для любого $\left.x \in \mathscr{O}_{v}\right\}$.

Пусть $L$ - поле функций некоторого $d$-мерного многообразия над $k, I \subseteq$ $\{1, \ldots, r\}, \mathscr{O}_{v} \in \mathscr{P}_{F}^{r}, p \in \mathscr{P}_{L}^{|I|}$. Пусть $O_{p, v, I}-$ множество всех вложений полей $\sigma: L \stackrel{/ k}{\hookrightarrow} F$ таких, что а) $\sigma^{-1}\left(\mathscr{O}_{v}\right)=\mathscr{O}_{p}$, б) $\sigma\left(L^{\times}\right) \cap \Gamma_{i} \neq \sigma\left(L^{\times}\right) \cap \Gamma_{i-1}$ тогда и только тогда, когда $1 \leqslant i \leqslant r$ и $i \in I$. 
ПРЕДЛОЖЕНИЕ 2.4.3. Если $m:=\max (0, r+d-n) \leqslant|I| \leqslant M:=\min (d, r), m o$ $O_{p, v, I}$ является непустой $G_{v}$-орбитой. Множество $\{L \stackrel{/ k}{\hookrightarrow} F\}$ вложений в $F$ над $k$ поля $L$ является несвязным обгединением орбит $O_{p, v, I}$. В частности,

$$
\mathbb{Q}[\{L \stackrel{/ k}{\hookrightarrow} F\}]_{G_{v}^{\dagger}}=\bigoplus_{s=m}^{M} \bigoplus_{p \in \mathscr{P}_{L}^{s}} \mathbb{Q}[\{\kappa(p) \stackrel{/ k}{\hookrightarrow} \kappa(v)\}]^{\left(\begin{array}{l}
r \\
s
\end{array}\right)}
$$

$u$

$$
G_{v} \backslash\{L \stackrel{/ k}{\hookrightarrow} F\}=\coprod_{s=m}^{M}\left(\mathscr{P}_{L}^{s}\right)^{\amalg}\left(\begin{array}{c}
r \\
s
\end{array}\right) .
$$

При $|I|=r$ стабилизаторь орбит $O_{p, v, I}$ изоморфны $\widehat{\mathbb{Z}}^{r} \times G_{\kappa(v) / \kappa(p)}($ икомпактны при $d=n$ ).

\section{Нормирования и максимальные подгруппы.}

ПримеР 2.4.4. При $n=1$ любому нормированию $v$ соответствует разложение $\{k(C) \stackrel{/ k}{\hookrightarrow} F\}=C(F) \backslash C(k)=\coprod_{C(k)}\left(\mathfrak{m}_{v} \backslash\{0\}\right)$.

ПреДЛОжеНИЕ 2.4.5. $G_{v}$ максималъна среди замкнутых подгрупп $G$ для любого $\mathscr{O}_{v} \in \mathscr{P}_{F}^{1}$.

В доказательстве проверяется, что для любой пары различных $\mathscr{O}_{v}, \mathscr{O}_{v^{\prime}} \in \mathscr{P}_{F}^{1}$ группа, порожденная $G_{v}$ и $G_{v^{\prime}}$, плотна в $G$, т. е. транзитивно действует на $\{L \stackrel{/ k}{\hookrightarrow} F\}$ для любого $L \mid k$ конечного типа. Вопрос сводится к случаю $n=1$, где $v$ и $v^{\prime}$ интерпретируются как согласованные наборы точек на "универсальной башне" кривых над $k$, после чего остается показать, что для любой пары различных точек $p, q \in C(k)$ на гладкой полной кривой $C$ над $k$ найдутся “этаж” $C_{\beta}$ этой башни и отображение $C_{\beta}$ в $C$, которое переводит пару $\left(v_{\beta}, v_{\beta}^{\prime}\right)$ B $(p, q)$.

Лемма 2.4.6. Групnа $G_{v}^{1}:=\left\{\sigma \in G_{v} \mid \sigma x / x \in 1+\mathfrak{m}_{v}\right.$ для любого $\left.x \in F^{\times}\right\} \subset G_{v}^{\dagger}$ дискретна при $n<\infty$.

При $n=r=1$ определим $\varphi: G_{v}^{1} \rightarrow \Gamma \cup\{+\infty\}$ формулой $\varphi(\sigma)=v(\sigma x / x-1)$ для любого $x \in \mathfrak{m}_{v} \backslash\{0\}$. Ясно, что $\varphi$ не зависит от $x$ и определяет ограниченное неархимедово биинвариантное расстояние на $G_{v}^{1}$. Логарифмическое расстояние преобразует присоединенное действие $G_{v}$ на $G_{v}^{1}$ в естественное действие $G_{v}$ на $\Gamma \cong \mathbb{Q}$. С помощью рядов Пюизё нетрудно показать, что отображение $G_{v}^{1}$ в себя, $\sigma \mapsto \sigma^{N}$, инъективно для любого $N \in \mathbb{N}$.

Пусть $G_{v}^{1}(\beta):=\left\{\sigma \in G_{v}^{1} \mid \varphi(\sigma) \geqslant \beta\right\}$, где $\beta \in \Gamma \otimes \mathbb{R}$. Это - нормальная подгруппа в $G_{v}^{\circ}$. Тогда $G_{v}^{1}=G_{v}^{1}(0)=G_{v}^{1}(0)^{+}$, где $G_{v}^{1}(\beta)^{+}:=\bigcup_{\gamma>\beta} G_{v}^{1}(\gamma)=\{\sigma \in$ $\left.G_{v}^{1} \mid \varphi(\sigma)>\beta\right\}$. Ясно, что $G_{v}^{1}(\beta) \neq G_{v}^{1}(\gamma)$, если $\beta \neq \gamma$. Группа $G_{v}^{1}(\beta)$ "очень" унипотентна. Например, имеется канонический изоморфизм $G_{v}^{1}(\beta) / G_{v}^{1}(\beta)^{+} \stackrel{\sim}{\longrightarrow}$ $\operatorname{Hom}\left(\Gamma, \mathfrak{m}^{[\beta]}\right)$, где

$$
\mathfrak{m}^{[\beta]}=\{x \in \mathfrak{m} \mid v(x) \geqslant \beta\} /\{x \in \mathfrak{m} \mid v(x)>\beta\} \cong \begin{cases}k, & \text { если } \beta \in \Gamma \text { и } \beta>0, \\ 0 & \text { иначе. }\end{cases}
$$

Лемма 2.4.7. Группа $G_{v}^{1}(\beta)$ сюргективна над $G / G^{\circ}$ для любого элемента $\beta \in \Gamma \otimes \mathbb{R}$. 
2.5. “Плотная" локально компактная "подгруппа" $\mathfrak{G}$ в $G$. Как известно из [1]-[4], в случае алгебраически замкнутого $F$ группа $G$ локально компактна тогда и только тогда, когда $n<\infty$. Пусть $n=\infty$ и $\left\{x_{1}, x_{2}, \ldots\right\}-$ базис трансцендентности поля $F$ над $k$. Положим $L_{m}:=k\left(x_{m}, x_{m+1}, \ldots\right) \subset F$. Тогда $L_{\bullet}=\left(L_{1} \supset L_{2} \supset L_{3} \supset \cdots\right)$ - убывающая последовательность подполей в $F$. Положим $\mathfrak{G}=\mathfrak{G}_{L \bullet}:=\bigcup_{m \geqslant 1} G_{F \mid L_{m}}$. Возьмем в качестве базиса открытых подгрупп множество $\left\{G_{F \mid L L_{1}}\right\}$, где $L$ пробегает подполя в $F$ конечного типа над $k$.

Геометрически (в смысле, аналогичном п. 3.4) это соответствует бесконечномерным многообразиям, заданным конечным числом уравнений, морфизмы между которыми не меняют координат с достаточно большими номерами.

Тогда:

- $\mathfrak{G}$ локально компактна (поскольку $F$ алгебраично над $\left.L_{1}\right)$, но не унимодулярна;

- включение $\mathfrak{G}$ в $G$ непрерывно и имеет плотный образ (это следует из равенства $\left.\bigcap_{m \geqslant 1} \bar{L}_{m}=k\right)$.

Чтобы описать модуль $\chi=\chi_{\mathfrak{G}}: \mathfrak{G} \rightarrow \mathbb{Q}_{+}^{\times}$, для каждого $\sigma \in G_{F \mid L_{m}} \subseteq \mathfrak{G}$ выберем подполе $L$ в $F \mid L_{m}$ конечного типа, над которым $F$ алгебраично, например, порожденное над $L_{m}$ некоторым базисом трансцендентности $F \mid L_{m}$. Тогда $\left[G_{F \mid L}\right]=\left[L \sigma(L): L^{\mathrm{insep}}\right] \cdot\left[G_{F \mid L \sigma(L)}\right]$ и $\left[G_{F \mid \sigma(L)}\right]=\left[L \sigma(L): \sigma(L)^{\mathrm{insep}}\right] \cdot\left[G_{F \mid L \sigma(L)}\right]$ для любого $\sigma \in G$, где - insep обозначает чисто несепарабельное замыкание в $L \sigma(L)$. Следовательно, $\chi(\sigma)=\left[L \sigma(L): \sigma(L)^{\mathrm{insep}}\right] /\left[L \sigma(L): L^{\mathrm{insep}}\right]$.

Для любого целого $q>1$ имеется элемент $\mathfrak{G}$, оставляющий неподвижными все элементы базиса трансцендентности $F \mid k$, кроме одного, $t$, на котором его действие имеет вид $t \mapsto t^{q}-t$. Поэтому $\chi$ сюръективен при всех $1 \leqslant n \leqslant \infty$.

В частности, группа $\mathfrak{G}$ не является компактно порожденной ни при каком $1 \leqslant n \leqslant \infty$, так как иначе группа значений модуля была бы конечно порождена, что неверно для $\mathbb{Q}_{+}^{\times}$.

Примеры гладких полулинейных представлений группы $\mathfrak{G}$. Скажем, что подмножества $I$ и $J$ в $\mathbb{N}$ соизмеримы, если $I \backslash(I \cap J)$ конечно и $|I \backslash(I \cap J)|=|J \backslash(I \cap J)|$. Обозначим через $[I]$ класс подмножеств в $\mathbb{N}$, соизмеримых с подмножеством $I$. Это - счетное множество.

Определим $\Omega_{F \mid k}^{[I]}$ как $F$-векторное пространство с базисом $\left\{d x_{j_{1}} \wedge d x_{j_{2}} \wedge\right.$ $\left.d x_{j_{3}} \wedge \cdots \mid J \in[I]\right\}$, где $J=\left(j_{1}<j_{2}<j_{3}<\cdots\right)$. Группа $\mathfrak{G}$ действует на $\Omega_{F \mid k}^{[I]}$ естественным образом. Если $I$ конечно мощности $q$, то получается представление $\Omega_{F \mid k}^{q}$. Если $I=\mathbb{N}$, то получается полулинейное представление степени 1 . Если $J=\mathbb{N} \backslash I$, то имеется невырожденное спаривание $\Omega_{F \mid k}^{[I]} \otimes_{F} \Omega_{F \mid k}^{[J]} \rightarrow \Omega_{F \mid k}^{[\mathbb{N}]}$, естественное, если зафиксировано $I \in[I]$. Из [12; лемма 7.7] следует, что полулинейное представление $\Omega_{F \mid k}^{[I]}$ неприводимо.

Пусть $M$ - множество таких отображений $f$ множества натуральных чисел в себя, что $\lim _{m \rightarrow \infty} f(m)=\infty$. Определим $\Omega_{F \mid k}^{M}$ как $F$-векторное пространство с базисом $\left\{d x_{f(1)} \otimes d x_{f(2)} \otimes d x_{f(3)} \otimes \cdots \mid f \in M\right\}$. Действие $\mathfrak{G}$ определяется естественным образом. Определим $\Omega_{F \mid k}^{[f]}$ как $F$-векторное подпространство в $\Omega_{F \mid k}^{M}$, натянутое на $\mathfrak{G}$-орбиту элемента $d x_{f(1)} \otimes d x_{f(2)} \otimes d x_{f(3)} \otimes \cdots$. 
2.6. Автоморфизмы группы $G$. Группа непрерывных автоморфизмов группы $G$ "имеет тот же размер", что и $G$. А именно, она совпадает с группой автоморфизмов поля $F$, сохраняющих алгебраически замкнутое подполе $k$.

При $n>1$ это утверждение следует из более сильного результата $\Phi$. А. Богомолова [27]-[29]: любой изоморфизм между абсолютными группами Галуа (и даже между их про-р-факторами по второму члену нижнего центрального ряда) полей функций многообразий над $k$ размерности $>1$ индуцирован изоморфизмом между этими полями. Основная часть доказательства в этом случае состоит в проверке того, что все абелевы подгруппы ранга $>1$ абсолютных групп Галуа содержатся в подгруппах разложения различных нормирований.

При $n=1$ это утверждение доказано в [30]. Общая идея состоит в следующем. Открытые компактные подгруппы $G^{\circ}$ - это абсолютные группы Галуа полей функций кривых над $k$ с отмеченной $F$-рациональной общей точкой. Эти кривые удается функториально описать в терминах топологической группы $G^{\circ}$ как проективные схемы над $\mathbb{Q}$. Например, абсолютные группы Галуа полей функций рациональных кривых над $k$ - это такие открытые компактные подгруппы $U$, что $N_{G}(U) / U$ бесконечна и не имеет абелевых подгрупп конечного индекса. В этом случае подгруппы разложения в $U^{\text {ab }}$ параметризованы множеством параболических подгрупп $P$ в $N_{G}(U) / U \cong \mathrm{PGL}_{2} k$ : подгруппа $D_{P} \cong \widehat{\mathbb{Z}}(1)$ состоит из элементов $U^{\mathrm{ab}}$, неподвижных относительно присоединенного действия $P$. Если открытая компактная подгруппа $U=G_{F \mid L}$ произвольна, то подгруппы разложения в $U^{\mathrm{ab}}$ можно описать как максимальные подгруппы в замыкании аддитивной оболочки образов гомоморфизмов переноса $G_{F \mid k(x)}^{\mathrm{ab}} \rightarrow G_{F \mid L}^{\mathrm{ab}}$ для всех $x \in L \backslash k$, проекции которых на $G_{F \mid k(x)}^{\mathrm{ab}}$ (относительно вложения $G_{F \mid L}^{\mathrm{ab}} \rightarrow \prod_{x \in L \backslash k} G_{F \mid k(x)}^{\mathrm{ab}}$, индуцированного включениями $\left.G_{F \mid L} \subseteq G_{F \mid k(x)}\right)$ являются подгруппами конечного индекса в некоторых подгруппах разложения в $G_{F \mid k(x)}^{\mathrm{ab}}$.

Из этого следует, что автоморфизм $G^{\circ}$ индуцирует автоморфизм всего набора кривых, т. е. автоморфизм поля $F$. Несколько более общая формулировка выглядит следующим образом.

Tеорема 2.6.1 [30; теорема 4.2]. Пусть $n=1$ u $H$ - nодгруnпа в $G$, coдеpжашая $G^{\circ}$. Тогда $N_{G_{F \mid \mathbb{Q}}}(H)$ содержится в группе $N_{G_{F \mid \mathbb{Q}}}(G)$ автоморфизмов поля $F$, сохраняющих подполе $k$, и присоединенное действие $N_{G_{F \mid \mathbb{Q}}}(H)$ на $H$ индущирует изоморфизм группы $N_{G_{F \mid \mathbb{Q}}}(H)$ на группу непрерывных открытых автоморфизмов $H$. Если $H \supseteq \operatorname{ker} \chi, \operatorname{mo} N_{G_{F \mid \mathbb{Q}}}(H)=N_{G_{F \mid \mathbb{Q}}}\left(G^{\circ}\right)$.

\section{3. Общие свойства гладких представлений группы $G$ и их реализации}

Прежде чем обсуждать представления $G$, сделаем несколько общих замечаний о категории $\mathscr{S} m_{H}(E)$ гладких $E$-представлений произвольной вполне несвязной топологической группы $H$.

ЗАмЕчаниЕ 3.0.1. Как известно (см., например, [31; доклад IV, §§ 2.4, 2.5] или $[32 ; \S 8.1$, пример 8.15 (iii)]), гладкие $H$-множества и их $H$-эквивариантные отображения образуют топос. 
Пусть $\mathfrak{T}=\mathfrak{T}(H, B)$ - категория, объекты которой - это элементы некоторой базы $B$ открытых подгрупп группы $H$, и $\operatorname{Hom}_{\mathfrak{T}}(U, V)=\{h \in H$ $\left.h U h^{-1} \supseteq V\right\} / U$. Композиция определяется естественным образом. Снабдим $\mathfrak{T}$ максимальной топологией, т. е. будем считать покрывающими все решета. Тогда пучки множеств, групп и т. п. отождествляются с гладкими $H$-множествами, группами и т. п.: $\mathscr{F} \mapsto \underset{U \in B}{\lim } \mathscr{F}(U)$ (это $H$-множество - гладкое, так как любой его элемент лежит в образе некоторого $\mathscr{F}(U)$, а действие $U$ на нем тривиально по определению) и $W \mapsto\left(U \mapsto W^{U}\right)$.

Например, если $B=\{1\}$ (и, в частности, $H$ дискретна), то в $\mathfrak{T}$ имеется ровно один объект $*$ и $\operatorname{Hom}_{\mathfrak{T}}(*, *)=H$.

Пусть $B$ - множество открытых подгрупп в $G$ вида $G_{F \mid L}$ (где $L \mid k$ - расширение конечного типа) и $\mathfrak{T}=\mathfrak{T}(G, B)$. Тогда

$$
\operatorname{Hom}_{\mathfrak{T}}\left(G_{F \mid L}, G_{F \mid K}\right)=\{h \in G \mid h(L) \subseteq K\} / G_{F \mid L}=\{h: L \stackrel{/ k}{\hookrightarrow} K\}
$$

- множество вложений полей над $k$. Когда $n=\infty$, лемма 3.4 .1 описывает гладкие $G$-множества как пучки на несколько ином сайте, в “доминантной топологии".

ЗАмечАниЕ 3.0.2. а) В категории $\mathscr{S} m_{H}(E)$ имеется достаточно много инъективных объектов. А именно, забывающий функтор $\mathscr{S} m_{H}(E) \rightarrow \mathrm{Vec}_{E}$ допускает правый сопряженный $I$ : для любого $E$-векторного пространства $V$ определим $I(V)$ как гладкую часть модуля $V$-значных функций на $H$, т. е. $I(V):=\underset{U \in B}{\lim _{U}} \operatorname{Maps}(H / U, V)$.

Группа $H$ действует на $I(V)$ сдвигами аргумента. Из полупростоты категории $E$-векторных пространств $\operatorname{Vec}_{E}$ следует инъективность $I(V)$. Если $V-$ гладкий $H$-модуль, то имеется $H$-эквивариантное вложение $V \rightarrow I(V), v \mapsto$ $(h \mapsto h v)$.

б) Объекты $E[H / U]$, где $U \in B$, задают систему образующих категории $\mathscr{S} m_{H}(E)$, т. е. любое гладкое циклическое $E$-представление $H$ является фактором представления $E[H / U]$ для некоторого $U \in B$. У представления $E[H / U]$ имеется не более $2^{\max (|H / U|,|E|)}$ факторов. Таким образом, у $H$ имеется не более $\max \left(|B|, \sup _{U} 2^{\max (|H / U|,|E|)}\right)$ циклических представлений. В случае $H=G$ получаем оценку $\leqslant 2^{\max (|k|,|E|)}$. Если $H$ локально компактна и не унимодулярна, то имеется не менее $\max \left(2^{\operatorname{rk}\left(\chi_{H}(H)\right)},|E|\right)$ неприводимых представлений. Например, если $n<\infty$, то в случае $H=G$ получаем оценку $\geqslant \max \left(2^{|\mathbb{N}|},|E|\right)$. Нижние оценки в случае $H=G$ приведены в предложении 3.5.2.

в) В категории $\mathscr{S} m_{H}(E)$ имеются прямые суммы, прямые произведения, тензорные произведения и внутренний функтор $\mathscr{H}$ om. Это - гладкие части соответствующих функторов на $\mathrm{Vec}_{E}$. А именно, прямое произведение семейства в $\mathscr{S} m_{H}(E)$ - это гладкая часть его теоретико-множественного прямого произведения, и

$$
\mathscr{H} o m\left(W_{1}, W_{2}\right):=\lim _{U} \operatorname{Hom}_{E[U]}\left(W_{1}, W_{2}\right)
$$


где $U$ пробегает открытые подгруппы в $H$. Функтор $\mathscr{H} o m(W,-)$ сопряжен справа к функтору $-\otimes_{E} W$ :

$$
\begin{aligned}
& \operatorname{Hom}_{\mathscr{S}_{m_{H}}(E)}\left(W_{1} \otimes_{E} W, W_{2}\right)=\operatorname{Hom}_{E[H]}\left(W_{1} \otimes_{E} W, W_{2}\right) \\
& =\operatorname{Hom}_{E[H]}\left(W_{1}, \operatorname{Hom}_{E}\left(W, W_{2}\right)\right)=\operatorname{Hom}_{E[H]}\left(W_{1}, \mathscr{H} o m\left(W, W_{2}\right)\right) \\
& =\operatorname{Hom}_{\mathscr{S}_{m_{H}}(E)}\left(W_{1}, \mathscr{H} \text { om }\left(W, W_{2}\right)\right)
\end{aligned}
$$

для любых $W_{1}, W_{2}, W \in \mathscr{S} m_{H}(E)$.

г) Если $\varphi: H_{2} \rightarrow H_{1}$ - гомоморфизм с плотным образом, то функтор обратного образа $\varphi^{-1}: \mathscr{S} m_{H_{1}} \rightarrow H_{2}$-mod - вполне строгий. (Доказательство. Пусть $W_{1}, W_{2} \in \mathscr{S} m_{H_{1}}, \alpha: \varphi^{*} W_{1} \rightarrow \varphi^{*} W_{2}-H_{2}$-гомоморфизм, $v \in W_{1}$ и $\sigma \in H_{1}$. Пусть $S$ - общий стабилизатор элементов $v$ и $\alpha(v)$. Выберем некоторый элемент $\sigma^{\prime} \in H_{2} \cap \varphi^{-1}(\sigma S)$. Тогда $\alpha(\sigma v)=\alpha\left(\sigma^{\prime} v\right)=\sigma^{\prime} \alpha(v)=\sigma \alpha(v)$.)

Если $\varphi$ непрерывен, то $\varphi^{-1}$ пропускается через $\varphi^{*}: \mathscr{S} m_{H_{1}} \rightarrow \mathscr{S} m_{H_{2}}$.

Если гомоморфизм $\varphi$ непрерывен и имеет плотный образ, то функтор $\varphi^{*}$ допускает правый сопряженный $\varphi_{*}: W \mapsto \bigcup_{U} W^{U \times_{H_{1}} H_{2}}$, где $U$ пробегает открытые подгруппы $H_{1}$. В частности, $\varphi^{*}$ сохраняет неприводимость. (Доказательство. Действие $H_{1}$ на $\varphi_{*} W$ определяется так. Если $w \in W^{\varphi^{-1}(U)}$ и $\sigma \in H_{1}$, то $\sigma w:=\sigma^{\prime} w$, где $\sigma^{\prime} \in H_{2}$ и $\varphi\left(\sigma^{\prime}\right) \in \sigma U$, что не зависит от $\sigma^{\prime}$.)

ПримеР 3.0.3. “Забывающий” функтор $\mathscr{S} m_{G} \rightarrow \mathfrak{G}$-mod вполне строг, сохраняет неприводимость, пропускается через $r: \mathscr{S} m_{G} \rightarrow \mathscr{S} m_{\mathfrak{G}}$, и $r$ допускает правый сопряженный: $W \mapsto \lim _{L} \bigcap_{m \geqslant 1} W^{G} F \mid L L_{m}$, где $L$ пробегает подполя конечного типа $F \mid k$.

ЗАмЕчАНИЕ 3.0.4. Если любая открытая подгруппа группы $H$ содержит открытую подгруппу бесконечного индекса (например, $H=G$ при $n=\infty$ ), то в категории гладких представлений группы $H$ нет ненулевых проективных объектов. (Доказательство. Пусть $W$ - проективный объект в категории гладких $E$-представлений группы $H$. Выберем систему образующих $\left\{e_{j}\right\}_{j \in J}$ представления $W$. Это задаст сюръективный гомоморфизм $\bigoplus_{j \in J} E\left[H / \mathrm{Stab}_{e_{j}}\right] \stackrel{\pi}{\rightarrow} W$. Зафиксируем элемент $i_{0} \in J$ и для каждого $j \in J$ зафиксируем открытую подгруппу $U_{j}$ бесконечного индекса в $\operatorname{Stab}_{e_{j}} \cap \mathrm{Stab}_{e_{i_{0}}}$. Поскольку $W$ проективен, композиция $\pi$ с сюръекцией $\bigoplus_{j \in J} E\left[H / U_{j}\right] \rightarrow \bigoplus_{j \in J} E\left[H / \mathrm{Stab}_{e_{j}}\right]$ расщепляется, и, следовательно, существует элемент в $\bigoplus_{j \in J} E\left[H / U_{j}\right]$ с тем же стабилизатором, что и $e_{i_{0}}$. Однако, поскольку $E\left[H / U_{j}\right]^{\mathrm{Stab}_{i_{0}}}=0$, отсюда следовало бы, что $e_{i_{0}}=0$, а значит, $W=0$.)

ЗАмечАние 3.0.5. а) Если $H$ локально компактна (например, $H=\mathfrak{G}$ для любого $n \leqslant \infty$, см. п. 2.5), то категория $\mathscr{S} m_{H}(E)$ имеет достаточно много проективных объектов. А именно, любой гладкий $H$-модуль является фактором прямой суммы объектов вида $E[H / U]$ для некоторых открытых компактных подгрупп $U$ группы $H$.

Однако $G$-модули $E[G / U]$ устроены очень сложно (см. п. 3.5). Кроме того (предложение 3.5.2), имеется "слишком много" $\left(\geqslant \max \left(2^{|\mathbb{N}|},|k|,|E|\right)\right)$ гладких неприводимых представлений группы $G$ для любого $1 \leqslant n \leqslant \infty$. Это - некоторые из причин изучать скорее категорию $\mathscr{I}_{G}$, см. стр. 92 (где есть надежда 
на бо́льшую контролируемость объектов, от которых ожидается, что они - "когомологической природы", см. п. 1.1), чем $\mathscr{S} m_{G}(E)$.

б) Категория $\mathscr{A} d m_{H}(E)$ допустимых представлений любой вполне несвязной группы $H$ замкнута относительно расширений и перехода к подобъектам в $\mathscr{S} m_{H}(E)$.

Если $H$ локально компактна, то $\mathscr{A} d m_{H}(E)$ - подкатегория Серра в $\mathscr{S} m_{H}(E)$.

ЗАмЕчАниЕ 3.0.6. Теория представлений локально компактных групп во многом определяется теорией представлений алгебр Гекке. Пусть, однако, $H$ произвольна.

Определим $\mathbb{D}_{E}(H):=\lim _{U} E[H / U]$, где $E-$ некоторое поле нулевой характеристики, а обратная система образована по отношению к проекциям $E[H / V] \stackrel{r_{V U}}{\longrightarrow} E[H / U]$ и $H / V \rightarrow H / U$, индуцированным включениями $V \subset U$ открытых подгрупп $H$. Для любых $\nu \in \mathbb{D}_{E}(H), \sigma \in H$ и открытой подгруппы $U$ пусть $\nu(\sigma U)$ - коэффициент при $[\sigma U]$ в образе $\nu$ в $E[H / U]$.

Для каждого гладкого $E$-представления $W$ группы $H$ определим спаривание $\mathbb{D}_{E}(H) \times W \rightarrow W$ формулой $(\nu, w) \mapsto \sum_{\sigma \in H / U} \nu(\sigma U) \cdot \sigma w$, где $U-$ произвольная открытая подгруппа стабилизатора элемента $w$, например, $U=\mathrm{Stab}_{w}$. Ясно, что результат не зависит от выбора $U$. Это определяет на $W$ структуру $\mathbb{D}_{E}(H)$ модуля. Когда $W=E[H / U]$, это спаривание согласовано с проекциями $r_{V U}$, так что оно задает спаривание $\mathbb{D}_{E}(H) \times \lim _{U} E[H / U] \rightarrow \lim _{U} E[H / U]=\mathbb{D}_{E}(H)$, а значит, и ассоциативное умножение $\mathbb{D}_{E}(H) \times \mathbb{D}_{E}(H) \stackrel{*}{\rightarrow} \mathbb{D}_{E}(H)$, продолжающее свертку мер с компактным носителем. (Носитель $\nu$ - минимальное замкнутое подмножество $S$ в полугруппе $\lim _{U} H / U$ такое, что $\nu(\sigma U)=0$ для $\sigma U$, не пересекающего $S$.)

Алгеброй Гекке пары $(H, U)$, где $U$ - компактная подгруппа в $H$, называется подалгебра $\mathscr{H}_{E}(H, U):=h_{U} * \mathbb{D}_{E}(H) * h_{U}$ в $\mathbb{D}_{E}(H) \quad U$-биинвариантных мер. Здесь $h_{U}$ - мера Хаара на $U$, определенная системой $\left(h_{U}\right)_{V}=[U$ : $U \cap V]^{-1} \sum_{\sigma \in U / U \cap V}[\sigma V] \in \mathbb{Q}[H / V]$ для всех открытых подгрупп $V \subset H$. Мера Хаара на $U$ - единица алгебры $\mathscr{H}_{E}(H, U)$ и $h_{U} h_{U^{\prime}}=h_{U}$ для замкнутой подгруппы $U^{\prime} \subseteq U$. Для каждого гладкого $E$-представления $W$ группы $H$ алгебра Гекке $\mathscr{H}_{E}(H, U)$ действует на пространстве $W^{U}$, поскольку $W^{U}=h_{U}(W)$.

Когда $H$ локально компактна, а $U$ открыта и компактна, это определение алгебры Гекке эквивалентно обычному определению и для каждого гладкого $E$-представления $W$ группы $H$ алгебра Гекке действует на пространстве $W^{U}$ обычным образом (см. [33]).

В случае $H=G$ и $n<\infty$ алгебры Гекке становятся алгебрами невырожденных соответствий на некоторых $n$-мерных многообразиях над $k$ (см. п. 3.1 , текст после леммы 3.1.3).

ЗАмЕчАНИЕ 3.0.7. Гладкие представления любой компактной группы полупросты. Пусть $U \subseteq H=G$ - компактная подгруппа, $\rho$ - ненулевое гладкое неприводимое представление $U$ над $\mathbb{Q}$ и $\sigma \in H$ - такой элемент, что $\sigma^{-1} H \sigma \subseteq H$. Определим представление $\rho^{\sigma}$ группы $U$ формулой $(\tau, u) \mapsto\left(\sigma^{-1} \tau \sigma\right) u$ для всех $\tau \in U$ и $u \in \rho$. Пусть $W$ - гладкое представление $G$. Тогда кратность $m_{W}(\rho) \geqslant 0$, с которой $\rho$ входит в $W$, равна $\operatorname{dim}_{\mathbb{Q}} \operatorname{Hom}_{U}(\rho, W) / \operatorname{dim}_{\mathbb{Q}} \operatorname{End}_{U}(\rho)$. 
Она конечна, если $W$ допустимо. Определим вложение $\operatorname{Hom}_{U}(\rho, W) \hookrightarrow$ $\operatorname{Hom}_{U}\left(\rho^{\sigma}, W\right)$ формулой

$$
\lambda \mapsto \sigma \lambda: \begin{array}{ccc}
u & \stackrel{\sigma \lambda}{\mapsto} & \sigma \lambda(u) \\
\downarrow & & \downarrow \tau \\
& \left(\sigma^{-1} \tau \sigma\right) u \stackrel{\sigma \lambda}{\mapsto} & \tau \sigma \lambda(u)
\end{array} .
$$

В частности, если $m_{W}(\rho) \neq 0$, то и $m_{W}\left(\rho^{\sigma}\right) \neq 0$. Ясно, что $\operatorname{End}_{U}(\rho) \subseteq \operatorname{End}_{U}\left(\rho^{\sigma}\right)$. Поэтому $m_{W}(\rho)=m_{W}\left(\rho^{\sigma}\right)$, если $\sigma^{-1} H \sigma=H$.

Заметим, что для любой пары компактных подгрупп $U_{1}, U_{2} \subset G$ любая пара их гладких представлений сплетена в обычном смысле, т. е. существуют $g \in G$ и ненулевой $\varphi \in \operatorname{Hom}\left(\rho_{1}, \rho_{2}\right)$ такие, что если $g k_{1}=k_{2} g$ и $k_{i} \in U_{i}, i \in\{1,2\}$, то $\varphi \rho_{1}\left(k_{1}\right)=\rho_{2}\left(k_{2}\right) \varphi$. А именно, существует такой $g \in G$, что $\left.\rho_{1}\right|_{U_{1} \cap g^{-1} U_{2} g}$ и $\left.\rho_{2}\right|_{U_{2} \cap g U_{1} g^{-1}}$ тривиальны (если $\rho_{i}$ конечной длины). Это объясняется тем, что представление $F$ (а значит, и неприводимое представление $F / k$ ) группы $G$ содержит все гладкие неприводимые (и значит, конечномерные) представления групп $U_{i}$ (теорема 90 Гильберта). По-видимому, то же верно и для $F^{\times} / k^{\times}$.

В [7; приложение А, теорема А.4] показано, что для любой компактной подгруппы $K$ в $G$ центры алгебр Гекке $\mathscr{H}_{E}(K)$ и $\mathscr{H}_{E}\left(G^{\circ}, K\right)$ пар $(G, K)$ и $\left(G^{\circ}, K\right)$ (определение $G^{\circ}$ см. в п.1.3) совпадают с $E \cdot h_{K}$, если $n<\infty$, т. е. состоят из скаляров. Это - отрицательные результаты, особенно в сравнении с аналогичными вопросами для $p$-адических групп.

В некоторых случаях группы морфизмов между геометрическими объектами отождествляются с группами морфизмов между соответствующими $G$-модулями (см. предложения 3.1.6 и 3.3.2 и следствие 3.1.7).

В [15] (см. следствие 3.4 .9 ниже) установлена бесконечность когомологических размерностей категорий $\mathscr{S} m_{G}$ и $\mathscr{C}$ (гладких полулинейных представлений $G$ ) при $n=\infty$.

По аналогии с соответствиями Лэнглендса, можно называть неприводимые представления группы $G$ в образе функтора $\mathfrak{B}^{n}$ каспидальными, где $\mathfrak{B}^{n}$ - некоторый функтор на категории примитивных $n$-мотивов, определенный (в большей общности) в разделе 4. Для групп GL над локальными неархимедовыми полями имеется несколько эквивалентных определений квазикаспидальных представлений. Одно из них (финитность): носители всех матричных коэфбфициентов гладкого представления $W$ топологической группы (т. е. функций на этой группе вида $\langle\sigma w, \widetilde{w}\rangle$ для некоторого вектора $w \in W$ и вектора с открытым стабилизатором $\widetilde{w}$ в двойственном представлении) компактны по модулю центра. Однако в [7; предложение 4.6] показано, что при $n<\infty$ все финитные представления любой подгруппы в $G$, содержащей $G^{\circ},-$ нулевые. Это выводится из неприводимости гладких представлений $F / k$ и или $F^{\times} / k^{\times}$подгруппы $G^{\circ}$ группы $G$ и их точности как модулей над соответствующими алгебрами мер на $G$ : для любого $1 \leqslant n \leqslant \infty$ аннулятор $F / k$ в $\mathbb{D}_{k}$ и аннулятор $F^{\times} / k^{\times}$в $\mathbb{D}_{\mathbb{Q}}$ тривиальны [7; предложение 4.2].

В случае $n=\infty$ можно установить некоторые аналоги теоремы 90 Гильберта. В частности, как вытекает из следствия 3.4 .8 , любой гладкий $G$-торсор над 
гладкой $G$-группой $B(F)$ тривиален для любой алгебраической $k$-группы $B$. Однако в случае $n<\infty$ имеются интересные примеры торсоров.

Согласно предложению 1.1.16, $\operatorname{Ext}_{\mathscr{S} m_{G}(\mathbb{Q})}(A(F) / A(k), \mathbb{Q})=\operatorname{Hom}(A(k), \mathbb{Q})$, если $n=\infty$ и $A$ - неприводимая коммутативная алгебраическая группа над $k$. Если $A$ - абелево многообразие, то $A(F) / A(k)=\mathfrak{B}^{1}\left(A^{\vee}\right)$, где $A^{\vee}:=\operatorname{Pic}^{\circ} A-$ двойственное абелево многообразие, а $\mathfrak{B}^{1}$ - функтор на категории примитивных 1-мотивов, определенный (в большей общности) в разделе 4. Поэтому это равенство естественно сравнить с тождеством $\operatorname{Ext}_{\mathcal{M} \mathcal{M}_{k}}^{1}\left(\mathbb{Q}(0), H_{1}(A)\right)=A(k)_{\mathbb{Q}}$ в категории смешанных мотивов над $k$.

Если $A=\mathbb{G}_{m}$, то тождество $\operatorname{Ext}_{\mathcal{M} \mathcal{M}_{k}}^{1}(\mathbb{Q}(0), \mathbb{Q}(1))=k^{\times} \otimes \mathbb{Q}$ подсказывает, что хотя гладкое представление $F^{\times} / k^{\times}$группы $G$ и не является допустимым, оно может иметь мотивную интерпретацию, аналогичную $\mathbb{Q}(1)$.

В п. 3.5 содержатся примеры пар различных расширений конечного типа $L_{1}$ и $L_{2}$ поля $k$ с одними и теми же наборами неприводимых подфакторов $\mathrm{JH}\left(L_{1}\right)$ и $\mathrm{JH}\left(L_{2}\right)$ представлений $\mathbb{Q}\left[\left\{L_{1} \stackrel{/ k}{\hookrightarrow} F\right\}\right]$ и $\mathbb{Q}\left[\left\{L_{2} \stackrel{/ k}{\hookrightarrow} F\right\}\right]$. В двух из этих примеров примитивные мотивы максимального уровня моделей полей $L_{1}$ и $L_{2}$ тривиальны, а в еще одном $L_{1}=k(X)$ и $L_{2}=k\left(\mathbb{P}^{\operatorname{dim} X}\right)$, где $X-$ произведение двулистных (в общей точке) накрытий проективных пространств (например, гиперэллиптических кривых) над $k$, из которых хотя бы одно - кривая рода $\leqslant 1$. Не исключено поэтому, что $\mathrm{JH}(L)$ зависит только от $\operatorname{deg} \operatorname{tr}(L \mid k)$.

Прежде всего установим некоторое обобщение того факта, что у $G$ нет конечномерных гладких представлений при $n=\infty$.

ПрЕДЛОЖЕНИЕ 3.0.8. Пусть $W \in \mathscr{S} m_{G}(E)$ u для некоторых подполей $\bar{L}_{1} \varsubsetneqq \bar{L}_{2}$ в $F \mid k$ подпространства $W^{G_{F \mid \bar{L}_{1}}} u W^{G_{F \mid \bar{L}_{2}}}$ - конечномерные и ненулевые. Тогда $W^{G}=W^{G_{F \mid \bar{L}_{1}}} \neq 0$. В частности, если $n=\infty u \operatorname{dim}_{E} W^{G_{F \mid \bar{L}}}<\infty$ для любого $L$ достаточно большой конечной степени трансцендентности над $k$, то $W$ тривиально.

ДокАзАТЕльство. Представление $W^{G_{F \mid \bar{L}_{1}}}$ группы $G_{\bar{L}_{1} \mid k}^{\circ}$ и представление $W^{G_{F \mid \bar{L}_{2}}}$ группы $G_{\bar{L}_{2} \mid k}^{\circ}$ тривиальны. Поэтому любой вектор $w \in W^{G_{F \mid \bar{L}_{1}}}$ неподвижен относительно действия подгруппы $H$ в $G$, порожденной подгруппами $G_{F \mid \bar{L}_{1}}\left(\right.$ в $\left.G_{\left\{F, \bar{L}_{1}\right\} \mid k} \times_{G_{\bar{L}_{1} \mid k}} G_{\bar{L}_{1} \mid k}^{\circ}\right)$ и $G_{\left\{F, \bar{L}_{2}\right\} \mid k} \times_{G_{\bar{L}_{2} \mid k}} G_{\bar{L}_{2} \mid k}^{\circ}$. Из леммы 2.2.6 следует, что эта подгруппа $H$ совпадает с $G$.

3.1. Алгебры Гекке и соответствия. Пусть $\mathscr{H}_{E}(U):=\mathscr{H}_{E}(G, U)=h_{U}$ * $\mathbb{D}_{E} * h_{U}$.

ПРЕДЛОЖЕНИЕ 3.1.1. 1) Пусть $H$ - вполне несвязная топологическая группа, а T - некоторое фильтрующее семейство ее компактных подгрупп, m.е. любая открытая подгруппа содержит элемент T. Тогда гладкое Е-представление $W$ группы $H$ неприводимо (соответственно полупросто) тогда и только тогда, когда $\mathscr{H}_{E}(H, U)$-модуль $W^{U}$ неприводим (соответственно полупрост) для каждой компактной подгруппъ $U \in T$.

2) Пусть $W_{j}$ для $j=1,2$ - гладкие неприводимые Е-представления $H$ u $W_{1}^{U} \neq 0$ для некоторой компактной подгруппь $U$. Тогда $W_{1}$ эквивалентно $W_{2}$ тогда и только тогда, когда $\mathscr{H}_{E}(H, U)$-модуль $W_{1}^{U}$ эквивалентен $W_{2}^{U}$. 
3) Для каждой открытой компактной подгруппы $U \subset H$ и каждого неприводимого Е-представления $\tau$ алгебры $\mathscr{H}_{E}(H, U)$ существует гладкое неприводимое представление $W$ группъ $H$ такое, что $\tau \cong W^{U}$.

Это предложение доказывается ровно так же, как предложение 2.10 в [33] и лемма 3.2 в [7].

Мы будем применять предложение 3.1 .1 в ситуации, когда $H=G$ и $T$ состоит из компактных подгрупп $U$ с подполем инвариантов $F^{U}$, чисто несепарабельным над чисто трансцендентным расширением расширения $k$ конечного типа.

ЗАМЕЧАНИЕ 3.1.2. Было бы естественно условия полупростоты или неприводимости представлений алгебр Гекке из предложения 3.1 .1 заменить соответствующими условиями на представления груnn $G_{F^{\prime} \mid k}$ для алгебраически замкнутых расширений $F^{\prime} \mid k$ в $F$ конечной степени трансцендентности. Для некоторого небольшого класса представлений это сделано в лемме 4.3.1. Следующая лемма - весьма предварительный шаг в общем направлении.

Лемма 3.1.3 [15]. Пусть $n=\infty$, $\mathscr{H}$ - подкатегория в $\mathscr{S} m_{G}$, замкнутая относительно перехода $\kappa$ подобгектам, и $F^{\prime} \mid k$ - алгебраически замкнутое расширение в F конечной степени трансцендентности. Следующие условия на подкатегорию $\mathscr{H}$ и $F^{\prime}$ эквивалентнъ:

1) для любого $W \in \mathscr{H}$ любой $G_{F^{\prime} \mid k}$-подмодуль $U \subseteq W^{G_{F \mid F^{\prime}}}$ совпадает $c G_{F^{\prime} \mid k}$-подмодулем $G_{F \mid F^{\prime}}$-инвариантов в $G$-подмодуле, порожденном $U$ : $U=\langle U\rangle_{G}^{G_{F \mid F^{\prime}}}$

2) для любого $W \in \mathscr{H}$ любая сюргекиия $\mathbb{Q}[\{L \stackrel{/ k}{\hookrightarrow} F\}]^{N} \rightarrow W$ в $\mathscr{S} m_{G}$

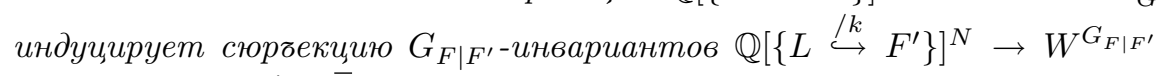
в $\mathscr{S} m_{F^{\prime} \mid k}$, где $F^{\prime}=\bar{L}$

3) для любого расширения $L \mid k$ в $F$ конечного типа, где $F^{\prime}=\bar{L}$, и любого $Q \subseteq \mathbb{Q}[\{L \stackrel{/ k}{\hookrightarrow} F\}]^{N}$ такого, что фактор лежит в $\mathscr{H}$, имеется равенство $H_{\mathscr{S} m_{G}}^{1}\left(G_{F \mid F^{\prime}}, Q\right)=0$.

Для любого неприводимого $k$-многообразия $Y$ с полем функций $k(Y)=F^{U}$ для некоторой компактной открытой подгруппы $U$ в $G$ можно отождествить алгебру Гекке $\mathscr{H}_{\mathbb{Q}}(U)$ с $\mathbb{Q}$-алгеброй невырожденных соответствий на $Y$ (т. е. формальных линейных комбинаций $n$-подмногообразий в $Y \times_{k} Y$, доминантных над обоими множителями $Y$ ). Это вытекает из следующей леммы и того, что

- множество двойных классов смежности $U \backslash G / U$ можно отождествить с базисом $\mathscr{H}_{\mathbb{Q}}(U)$ как $\mathbb{Q}$-векторного пространства с помощью $[\sigma] \mapsto h_{U} *$ $\sigma * h_{U}$

- неприводимые $n$-подмногообразия в $Y \times_{k} Y$, доминантные над обоими множителями $Y$, находятся в естественной биекции с множеством максимальных идеалов алгебры $F^{U} \otimes_{k} F^{U}$.

ЛЕмма 3.1.4 [7; лемма 3.3]. Пусть $L, K \subseteq F$ - расширения поля $k$, причем $\operatorname{deg} \operatorname{tr}(L \mid k)=q<\infty$. Тогда множество $G_{F \mid K} \backslash G / G_{F \mid L}$ двойных смежных классов канонически отождествляется с множеством всех точек $\operatorname{Spec}\left(L \otimes_{k} K\right)$ 
коразмерности $\geqslant q-\operatorname{deg} \operatorname{tr}(F \mid K)\left(\operatorname{ma\kappa }\right.$ что $G_{F \mid K} \backslash G / G_{F \mid L}=\operatorname{Max}\left(L \otimes_{k} K\right)$, если $F=\bar{K})$. Здесъ $G / G_{F \mid L}$ - множество вложений поля $L$ в $F$ над $k$.

Пусть $A^{q}(Y)$ - фактор $\mathbb{Q}$-векторного пространства $Z^{q}(Y)$ циклов на гладком собственном многообразии $Y$ над $k$ коразмерности $q$ по $\mathbb{Q}$-векторному подпространству $Z_{\sim}^{q}(Y)$ циклов, $\sim$-эквивалентных нулю для некоторого адекватного отношения эквивалентности $\sim$. Согласно теореме Хиронаки о разрешении особенностей, каждое гладкое многообразие $X$ допускает открытое вложение $i$ в гладкое собственное многообразие $\bar{X}$ над $k$. Тогда $A^{q}(\cdot)$ можно продолжить на произвольное гладкое многообразие $X$ как коядро отображения $Z_{\sim}^{q}(\bar{X}) \stackrel{i^{*}}{\longrightarrow} Z^{q}(X)$, индуцированного ограничением циклов. Это не зависит от выбора многообразия $\bar{X} .^{3}$

Стандартным образом, можно продолжить контравариантные функторы $A^{q}(\cdot)$ и $Z^{q}(\cdot)$ до контравариантных функторов на категории гладких промногообразий над $k$. А именно, если для некоторого множества индексов $I$ обратная система $\left(X_{j}\right)_{j \in I}$ гладких многообразий над $k$ образована по отношению к плоским морфизмам и $X$ - ее предел, то $Z^{q}(X)=\underset{j \in I}{\lim } Z^{q}\left(X_{j}\right)$, где индуктивная система образована по отношению к обратным образам, и аналогично для $A^{q}(\cdot)$. Тогда $A^{q}(X)$ - коядро отображения $\bigoplus_{j \in I} Z_{\sim}^{q}\left(\overline{X_{j}}\right) \rightarrow Z^{q}(X)$. Это не зависит от выбора проективной системы, определяющей $X$.

В частности, поскольку для любой коммутативной $k$-алгебры $R$ схема $\operatorname{Spec}(R)$ - обратный предел некоторой системы $k$-многообразий, определено $A^{q}(R):=A^{q}(\mathbf{S p e c}(R))$. Любой автоморфизм $\alpha \quad k$-алгебры $R$ индуцирует морфизм системы $\left(X_{j}\right)_{j \in I}$, определяющей $\operatorname{Spec}(R)$, в систему $\left(\alpha^{*}\left(X_{j}\right)\right)_{j \in I}$, канонически эквивалентную системе $\left(X_{j}\right)_{j \in I}$, и, следовательно, индуцирует автоморфизм $A^{q}\left(Y_{R}\right)$ для любой $k$-схемы $Y$. Это определяет контравариантный функтор из категории многообразий над $k$ в категорию $\operatorname{Aut}(R \mid k)$-модулей.

В нашей ситуации $X$ будет иметь вид $Y_{F}$ для некоторой $k$-подсхемы $Y$ в некотором многообразии над $k$.

Гомоморфизм алгебр $\mathscr{H}_{\mathbb{Q}}(U) \rightarrow A^{\operatorname{dim} Y}\left(Y \times_{k} Y\right)$ сюръективен для любого гладкого проективного $Y$, где $k(Y) \subset F$ и $U=G_{F \mid k(Y)}$. Это видно из следующей “леммы о сдвиге", примененной в случае $X_{1}=X_{2}=Y$ и $Z=X_{1} \times_{k} X_{2}$ (и приводимой в форме, предложенной рецензентом).

Лемма 3.1.5 [7; лемма 3.4]. Пусть $Z, X_{1}, \ldots, X_{r}$ - неприводимъье проективные многообразия над $k$, и пусть $Z \stackrel{p_{j}}{\longrightarrow} X_{j}$ - сюргективные отображения. Пусть $q \geqslant \max _{1 \leqslant j \leqslant r} \operatorname{dim} X_{j} u \alpha \subset Z$ - неприводимое подмногообразие размерности $q$. Тогда $\alpha$ рационально эквивалентен линейной комбинации некоторьх неприводимых подмногообразий в $Z$, сюргективных (относителъно отображений $p_{j}$ ) надо всеми $X_{j}$.

${ }^{3}$ Поскольку любая пара гладких компактификаций $\left(\bar{X}, \bar{X}^{\prime}\right)$ многообразия $X$ доминируется третьей $\bar{X} \stackrel{\beta}{\longleftarrow} \bar{X}^{\prime \prime} \stackrel{\beta^{\prime}}{\longrightarrow} \bar{X}^{\prime}, i^{*}$ пропускается через $Z_{\sim}^{q}(\bar{X}) \stackrel{\beta^{*}}{\longrightarrow} Z_{\sim}^{q}\left(\bar{X}^{\prime \prime}\right) \stackrel{\left(i^{\prime \prime}\right)^{*}}{\longrightarrow} Z^{q}(X)$ и $i^{*} Z_{\sim}^{q}(\bar{X})=\left(i^{\prime \prime}\right)^{*} Z_{\sim}^{q}\left(\bar{X}^{\prime \prime}\right)$. 
В частности, естественная проекиия $Z^{q}\left(k(X) \otimes_{k} k(Y)\right) \rightarrow C H^{q}\left(X \times_{k} Y\right)$ сюргективна при $q \leqslant \operatorname{dim} X \leqslant \operatorname{dim} Y$, a $Z^{q}\left(k(Y) \otimes_{k} F\right) \rightarrow C H^{q}\left(Y_{F}\right)$ сюргективна при $q \leqslant \operatorname{dim} Y \leqslant n$.

ПРЕДЛОЖЕНИЕ 3.1.6. Пусть $Y$ - гладкое неприводимое собственное многообразие над $k u \operatorname{dim} Y \leqslant n$. Пусть $X-$ гладжое многообразие над $k u W$ фактор представления $A^{q}\left(X_{F}\right)$ группы $G$ для некоторого $q \geqslant 0$. Тогда имеются канонические изоморфизмы $A^{q}\left(X_{k(Y)}\right) \stackrel{\sim}{\longrightarrow} \operatorname{Hom}_{G}\left(A^{\operatorname{dim} Y}\left(Y_{F}\right), A^{q}\left(X_{F}\right)\right)$ u $\operatorname{Hom}_{G}\left(A^{\operatorname{dim} Y}\left(Y_{F}\right), W\right) \stackrel{\sim}{\longrightarrow} \operatorname{Hom}_{G}\left(Z^{\operatorname{dim} Y}\left(k(Y) \otimes_{k} F\right), W\right)$.

Это предложение фактически не отличается от предложения 3.6 в [7]. Его доказательство использует леммы 3.1.4 и 3.1.5 и элементарную теорию пересечений.

СлЕДСТвИЕ 3.1 .7 [7; следствие 3.7]. Для любого поля $L^{\prime}$, конечно порожденного и степени трансцендентности $m \leqslant n$ над $k$, любого поля $L$, конечно порожденного над $k$, июбого иелого $q \geqslant m$ имеется канонический изоморфизм $A^{q}\left(L \otimes_{k} L^{\prime}\right) \stackrel{\sim}{\longrightarrow} \operatorname{Hom}_{G}\left(A^{m}\left(L^{\prime} \otimes_{k} F\right), A^{q}\left(L \otimes_{k} F\right)\right)$, где обе группь нулевъе при $q>m$.

\section{2. Инварианты подгрупп и тензорные произведения.}

Лемма 3.2.1. Пусть $W$ - гладкое $G$-множество и $L$-расширение $k$ в $F$. Тогда $W^{G_{F \mid L}}=\bigcup_{L_{0} \subseteq L} W^{G_{F \mid L_{0}}}$, где $L_{0}$ пробегает расширения $k$ конечного типа.

Доказательство идентично доказательству леммы 6.1 работы [7].

ПреДЛОЖЕНИЕ 3.2.2. Пусть $E-$ - ил $F$, или любое поле характеристики нуль с тривиальным действием $G$ и $W_{1}, W_{2}$ - гладкие полулинейные представления $G$ над $E$. Предположим, что либо подгруппа $H \subseteq G$ не допускает нетривиальных конечномерных гладких полулинейных представлений над $E$, либо $\operatorname{deg} \operatorname{tr}(F \mid k)=\infty$ и $H=G_{F \mid \bar{L}}$ для некоторого расширения $L \subset F$ поля $k$. Тогда имеется равенство $\left(W_{1} \otimes_{E} W_{2}\right)^{H}=W_{1}^{H} \otimes_{E^{H}} W_{2}^{H}$.

"Обратно", если $\left(W_{1} \otimes_{E} W_{2}\right)^{H}=W_{1}^{H} \otimes_{E^{H}} W_{2}^{H}$ для некоторой подгруппь $H \subset G$, то поле $F^{H}$ алгебраччески замкнуто.

Если $\operatorname{deg} \operatorname{tr}(F \mid k)<\infty$ и $H$ имеет вид $G_{F \mid \bar{L}}$, то равенство $\left(W_{1} \otimes_{E} W_{2}\right)^{H}=$ $W_{1}^{H} \otimes_{E^{H}} W_{2}^{H}$, вообще говоря, не выполнено. А именно, если $W_{1}$ и $W_{2}-$ нетривиальные одномерные представления группы $G$, двойственные друг другу, то $\left(W_{1} \otimes_{E} W_{2}\right)^{G}=E^{G}$, но $W_{1}^{G}=W_{2}^{G}=0$.

Это предложение - небольшое обобщение леммы 7.5 работы [12], доказывается аналогично.

3.3. $G$ - и $G^{\circ}$-модули вида $A(F) / A(k)$, где $A$ - коммутативная $k$-группа, морфизмы между ними и сепарабельное замыкание одномерного расширения $k$.

ПРЕДЛОЖЕНИЕ 3.3.1. Если $n=1$, mo $G^{\circ}$-орбита $x$ порождает сепарабельное замыкание $K_{x}$ поля $k(x)$ в $F$ для любого $x \in F \backslash k$. Точнее, $K_{x}^{\times} / k^{\times}-$ неприводимыи $G^{\circ}$-модуль, а $K_{x} / k$ - неприводимый $G^{\circ}$-модуль при $\operatorname{char}(k) \neq 2$.

$G^{\circ}$-модули $F / k$ и $F^{\times} / k^{\times}$неприводимь, если характеристика $k$ - нулевая uлu $2 \leqslant n \leqslant \infty$. 
ДоказАтельство. В [7; предложение 4.1] показано, что если $A$ - подгруппа аддитивной группы поля $F$, порожденная $G^{\circ}$-орбитой некоторого $x \in F \backslash k$, и характеристика $k$ отлична от 2 , то $A$ - подполе в $F$. Кроме того, если $M-$ подгруппа мультипликативной группы $F^{\times}$поля $F$, порожденная $G^{\circ}$-орбитой $x$, то $M \cup\{0\}-G^{\circ}$-инвариантное подполе в $F$.

Очевидно, что $A=M \cup\{0\}=F$ при $n \geqslant 2$. Если $n=1, \operatorname{to} \operatorname{Gal}\left(F \mid \mathbb{Q}\left(G^{\circ} x\right)\right)-$ компактная нормальная, т. е. - тривиальная (теорема 2.2.1), подгруппа в $G^{\circ}$. Таким образом, расширение $F \mid \mathbb{Q}\left(G^{\circ} x\right)$ чисто несепарабельно.

Покажем, что $k\left(G^{\circ} x\right)$ - сепарабельное расширение поля $k(x)$. Другими словами, что если $\sigma^{N} x=x$ для некоторого $N \geqslant 1$, то $k(x, \sigma x)$ - сепарабельное расширение поля $k(x)$. Пусть $P(x, \sigma x)$ - минимальный многочлен. Тогда $P_{\mathrm{I}} d x+P_{\mathrm{II}} d(\sigma x)=0 \in \Omega_{k(x, \sigma x) \mid k}^{1}$, где или $P_{\mathrm{I}} \neq 0$, или $P_{\mathrm{II}} \neq 0$, так как иначе $P=Q^{p}$ для другого многочлена $Q$. Если $P_{\text {II }} \neq 0$, то $k(x, \sigma x)$ - сепарабельное расширение поля $k(x)$. Если $P_{\mathrm{I}} \neq 0$, то $k(x, \sigma x)$ - сепарабельное расширение поля $k(\sigma x)$ и, значит, $k\left(x, \sigma^{-1} x\right)$ - сепарабельное расширение поля $k(x)$. Тогда поле, порожденное над $k$ элементами $x, \sigma^{-1} x, \ldots, \sigma^{-(N-1)} x=\sigma x,-$ сепарабельное расширение поля $k(x)$.

ПредлОЖЕНИЕ 3.3.2 [7; предложения 3.6, 4.3]. Пусть $1 \leqslant n \leqslant \infty u A$ u $B$ приведенные неприводимые групповые $k$-схемы и пусть $\operatorname{Hom}(A, B)$ - множество гомоморфизмов групповых $k$-схем. Тогда естественное отображение $\operatorname{Hom}(A, B) \rightarrow \operatorname{Hom}_{G}(A(F), B(F))$, где $\operatorname{Hom}_{G}-G$-гомоморфизмы, является биекиией.

Допустим, что k-группь $A$ и В коммутативны и просты. Тогда

$$
\begin{aligned}
\operatorname{Hom}(A, B)_{\mathbb{Q}} & \stackrel{\sim}{\longrightarrow} \operatorname{Hom}_{G}(A(F) / A(k), B(F) / B(k)) \\
& \stackrel{\sim}{\longrightarrow} \operatorname{Hom}_{G^{\circ}}(A(F) / A(k), B(F) / B(k)) .
\end{aligned}
$$

Доказательство второй части состоит, к сожалению, из перебора случаев: $A$ и $B$ - простые абелевы многообразия, $\mathbb{G}_{a}$ или $\mathbb{G}_{m}$.

3.4. Доминантная топология, ацикличность некоторых из гладких представлений $G$ и бесконечность когомологической размерности. (Основные результаты этого пункта взяты из [15].) В этом пункте мы отождествляем гладкие представления с абелевыми пучками на некотором "малом" сайте и интерпретируем гладкие когомологии (т. е. $\left.\operatorname{Ext}_{\mathscr{S} m_{G}}^{*}(\mathbb{Q},-)\right)$ как когомологии Чеха пучков.

Мы будем считать, что $F \mid k$ - расширение алгебраически замкнутых полей нулевой характеристики счетной степени трансцендентности.

Пусть $\mathfrak{D} m_{k}-$ категория гладких морфизмов гладких $k$-схем. Снабдим $\mathfrak{D} m_{k}$ предтопологией, в которой покрытия - это доминантные морфизмы.

Лемма 3.4.1. Категория пучков на $\mathfrak{D} m_{k}$ эквивалентна категории гладких G-множеств.

Пучку сопоставляется его “общий слой", т. е. $\underset{U \longrightarrow}{\longrightarrow} \mathscr{F}(U)$, где $U$ пробегает множество гладких целых $k$-многообразий с полем функций, вложенным в $F$ над $k$. 
ПРЕДЛОЖЕНИЕ 3.4.2. Пусть степень трансцендентности расширения $F^{\prime} \mid k$ в $F$ бесконечна. Тогда $H_{\mathscr{S} m_{G}}^{>0}\left(G_{F \mid \overline{F^{\prime}}}, W\right)=H_{\mathscr{S} m_{G}}^{>0}\left(G_{F \mid F^{\prime}}, W \otimes \mathbb{Q}\right)=0$ для любого гладкого $G$-модуля $W$.

\section{Когомологии Чеха.}

Лемма 3.4.3. Комплекс $\cdots \rightarrow \mathbb{Q}\left[\left\{L\left(Y^{2}\right) \stackrel{/ k}{\hookrightarrow} F\right\}\right] \rightarrow \mathbb{Q}[\{L(Y) \stackrel{/ k}{\hookrightarrow} F\}] \rightarrow$ $\mathbb{Q}[\{L \stackrel{/ k}{\hookrightarrow} F\}] \rightarrow 0$ ацикличен для любого L-многообразия $Y$. Eсли $L=k$ и $Y$ - гладкое собственное, то комплексъ $\cdots \rightarrow C_{k\left(Y^{2}\right)} \rightarrow C_{k(Y)} \rightarrow \mathbb{Q} \rightarrow 0$ u $\cdots \rightarrow C H_{0}\left(Y^{2}\right)_{\mathbb{Q}} \rightarrow C H_{0}(Y)_{\mathbb{Q}} \rightarrow \mathbb{Q} \rightarrow 0$ также ацикличны.

СледСтВиЕ 3.4.4. Для любого $W \in \mathscr{I}_{G}$ комплекс $0 \rightarrow W^{G} \rightarrow W^{G_{F \mid k(Y)}} \rightarrow$ $W^{G_{F \mid k\left(Y^{2}\right)}} \rightarrow \cdots$ точен. $\quad$ В частности, $0 \rightarrow C H^{q}(X)_{\mathbb{Q}} \rightarrow C H^{q}\left(X_{k(Y)}\right)_{\mathbb{Q}} \rightarrow$ $C H^{q}\left(X_{k\left(Y^{2}\right)}\right)_{\mathbb{Q}} \rightarrow \cdots$ точен.

Поскольку $\mathscr{I}$ точен справа, из леммы 3.4 .3 получаем следующее утверждение.

СлЕДСТВИЕ 3.4.5. Отображение поля функций $L$ многообразия над $k$ в $\mathbb{Q}[\{L \stackrel{/ k}{\hookrightarrow} F\}] \in \mathscr{S} m_{G}\left(\right.$ соответственно в $\left.C_{L} \in \mathscr{I}_{G}\right)$ определяет пучок на $\mathfrak{D} m_{k}$ со значениями в $\mathscr{S}_{G}^{\text {op }}\left(\right.$ соответственно в $\left.\mathscr{I}_{G}^{\mathrm{op}}\right)$.

Обозначим через $\check{H}^{*}$ когомологии Чеха.

СледСтвиЕ 3.4 .6 [34; гл. III, следствие 2.5]. $\check{H}^{*}$ совпадает с $H^{*}$ для любого пучка тогда и только тогда, когда $\check{H}^{*}$ переводит любую короткую точную последовательность пучков в длинную точную последовательность когомологий Чеха.

Для любого подполя $K$ в $F \mid k$, над которым степень трансцендентности $F$ бесконечна, зафиксируем базис трансцендентности $\left\{x_{1}, x_{2}, x_{3}, \ldots\right\}=\left\{x_{1}^{(\bar{K})}\right.$, $\left.x_{2}^{(\bar{K})}, x_{3}^{(\bar{K})}, \ldots\right\}$ поля $F$ над $K$.

Для каждого $m \geqslant 0$ положим $F_{m}=\overline{K\left(x_{2^{m}}, x_{2^{m} \cdot 3}, x_{2^{m} \cdot 5}, \ldots\right)}$ и для каждого $j \geqslant 0$ зафиксируем вложение $\sigma_{j}$ поля $F$ в себя над $\bar{K}$ такое, что $\left.\sigma_{j}\right|_{F_{s}}=\mathrm{id}$, если $j>s$, и $\left.\sigma_{j}\right|_{F_{s}}=\left.\sigma_{s}\right|_{F_{s}}: F_{s} \stackrel{\sim}{\longrightarrow} F_{s+1}$, если $j \leqslant s$. Для любого расширения $L$ поля $K$ зафиксируем $L_{0} \subseteq F_{0}$, изоморфное над $K$ полю $L$, и положим $L_{s}:=$ $\sigma_{0}^{s}\left(L_{0}\right)$.

Для $G$-модуля $W$ положим

$$
{ }_{K}^{L} W^{\bullet}:=\left(W^{G_{F \mid L_{0}}} \stackrel{\sigma_{0}-\sigma_{1}}{\longrightarrow} W^{G_{F \mid L_{0} L_{1}}} \stackrel{\sigma_{0}-\sigma_{1}+\sigma_{2}}{\longrightarrow} W^{G_{F \mid L_{0} L_{1} L_{2}}} \rightarrow \cdots\right) .
$$

ПРЕДЛОЖЕНИЕ 3.4.7. $H_{\mathscr{S} m_{G}}^{q}\left(G_{F \mid K}, W\right)=H^{q}\left(\underset{K}{F} W^{\bullet}\right)$ для любого гладкого представления $W$ группь $G$ и любого алгебраччески замкнутого расширения $K \supseteq k$ \& $F$.

Ацикличность "геометрических" $G$-модулей и когомологическая размерность категории $\mathscr{S} m_{G}$. Сопоставим предпучку $\mathscr{F}$ на $\mathfrak{D} m_{k}$ и фильтрованному объединению $\mathscr{O}=\lim _{U} A$ конечно порожденных гладких $k$-подалгебр $A$ гладкое $\operatorname{Aut}(\mathscr{O} \mid k)$-множество $\mathscr{F}(\mathscr{O}):=\lim _{A} \mathscr{F}(\operatorname{Spec}(A))$. В качестве $\mathscr{O}$
будут выступать $F$ и $\mathscr{O}_{v}$. 
Покажем, что $\mathscr{F}(F)^{G_{F \mid F^{\prime}}}=\mathscr{F}\left(F^{\prime}\right)$ для любого $F^{\prime}=\overline{F^{\prime}} \subseteq F \mathrm{c} \operatorname{deg} \operatorname{tr}\left(F^{\prime} \mid k\right)=\infty$.

(Как показывает пример предпучка $\mathscr{F}: U \mapsto \Gamma\left(\bar{U}, \bigotimes_{\mathscr{O}}^{2} \Omega_{\bar{U} / k}^{1}\right)$, условие $F^{\prime}=\overline{F^{\prime}}$ существенно.)

Зафиксируем изоморфизм $\alpha: F \stackrel{\sim}{\longrightarrow} F^{\prime}$ над $k$. Он индуцирует изоморфизм $\alpha^{*}: \lim _{U} \mathscr{F}(U) \stackrel{\sim}{\longrightarrow} \lim _{V} \mathscr{F}(V)$, где $\mathscr{O}(U) \subset F$ и $\mathscr{O}(V) \subset F^{\prime}$ пробегают гладкие $k$-подалгебры. Для любого $U$ существует $\sigma \in G$ такое, что $\left.\sigma\right|_{\mathscr{O}(U)}=\left.\alpha\right|_{\mathscr{O}(U)}$, так что $\lim _{V} \mathscr{F}(V)=\underset{(U, \sigma)}{\lim } \sigma \mathscr{F}(U)=\mathscr{F}(F)^{G_{F \mid F^{\prime}}}$, cр. с леммой 4.1.2.

Допустим, что для любых гладких многообразий $X, Y$ и любой точки $x \in$ $X(k)$ предпучок $\mathscr{F}$ снабжен преобразованиями $i_{X, Y}^{x}: \mathscr{F}\left(X \times_{k} Y\right) \rightarrow \mathscr{F}(Y)$ таки-

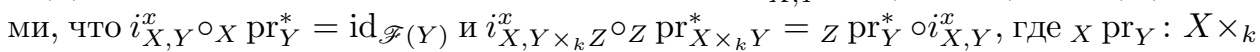
$Y \rightarrow Y-$ проекция.

Пусть $\mathscr{R}$ - предпучок коммутативных колец и $\mathscr{F}-\mathscr{R}$-модуль. Тогда представление $\mathscr{F}(F)$ является $\mathscr{R}(F)$-модулем и такие представления (для всевозможных $\mathscr{F}$ при фиксированном $\mathscr{R}$ ) образуют тензорную категорию по отношению к операции $\otimes_{\mathscr{R}(F)}$. В частности, категория представлений вида $\mathscr{F}(F)$ для $\mathscr{F}$ со значениями в коммутативных группах - тензорная.

СлеДСТвиЕ 3.4 .8 [15]. $H_{\mathscr{S}_{m}}^{>0}(G, W)=0$ для любого $W \in \mathscr{I}_{G} ;$ для любого $\mathscr{F}$ со значениями в группах множество $H_{\mathscr{S} m}^{1}(G, \mathscr{F}(F))$ состоит из единственного элемента; $H_{\mathscr{S} m}^{>0}(G, \mathscr{F}(F))=0$ для любого $\mathscr{F}$ со значениями в коммутативных группах.

Примеры представлений вида $\mathscr{F}(F)$ как в следствии 3.4 .8 дают $A(F)$ для любого группового $k$-многообразия $A$, или $A(F) / A(k)$, если $A$ коммутативно, $C H_{0}\left(X_{F}\right)_{\mathbb{Q}}, \Omega_{F \mid k, \text { рег }}^{\bullet}, \bigotimes_{F}^{\bullet} \Omega_{F \mid k_{0}}^{1}, \Omega_{F \mid k_{0}, \text { замкн }}^{\bullet}, \Omega_{F \mid k_{0}, \text { точн }}^{\bullet}$ и $H_{\mathrm{dR} / k_{0}}^{\bullet}(F)$ для любого $k_{0} \subseteq k$. (Соответствующие функторы: $A(\mathscr{O})$, или $A(\mathscr{O}) / A(k), C H_{0}\left(X \times_{k}(-)\right)_{\mathbb{Q}}$, $\Gamma\left(\tau, \Omega_{\mathscr{O} \mid k}^{\bullet}\right), \bigotimes_{\mathscr{O}}^{\bullet} \Omega_{\mathscr{O} \mid k_{0}}^{1}, \Omega_{\mathscr{O} \mid k_{0}, \text { замкн }}^{\bullet}, \Omega_{\mathscr{O} \mid k_{0}, \text { точн }}^{\bullet}$ и $\left.H_{\mathrm{dR} / k_{0}}^{\bullet}(\mathscr{O}).\right)$

СлеДСтвие 3.4 .9 [15]. 1) Категории $\mathscr{S} m_{G} u \mathscr{C}$ (см. п. 1.2) допускают системы ацикличных образующих $\{\mathbb{Q}[\{L \stackrel{/ k}{\hookrightarrow} F\}]\}_{L} \quad u\{F[\{L \stackrel{/ k}{\hookrightarrow} F\}]\}_{L}$, где $L-$ поля конечного типа над $k$, содержащие любое фиксированное расширение $k$ конечного типа.

2) Когомологические размерности категорий $\mathscr{S}_{G}$ и $\mathscr{C}$ бесконечны.

$\mathbb{A}^{1}$-инвариантность некоторых предпучков. Пусть $\mathscr{V}_{k}$ - некоторая категория многообразий над $k$, содержащая все гладкие многообразия. Пусть $\mathscr{L}-$ некоторая категория, в которой все вложения всех объектов в себя являются изоморфизмами (например, такая абелева категория, объекты которой исчерпываются подобъектами конечной длины, что для любого объекта кратности его неприводимых подфакторов конечны).

$\mathscr{L}$-значный предпучок $\mathscr{F}$ на $\mathscr{V}_{k}$ называется $\mathbb{A}^{1}$-инвариантным, если $\mathscr{F}(U)=$ $\mathscr{F}\left(U \times \mathbb{A}^{1}\right)$ для любого $U \in \mathscr{V}_{k}$.

Рассмотрим любую предтопологию на $\mathscr{V}_{k}$ такую, что морфизмы $k$-схем $\mathbb{A}_{k}^{1} \rightarrow$ $\operatorname{Spec} k, \quad\left(\mathbb{A}_{k}^{1} \backslash\{0\}\right) \coprod\left(\mathbb{A}_{k}^{1} \backslash\{1\}\right) \rightarrow \mathbb{A}_{k}^{1}$ и $\mathbb{G}_{m, k} \rightarrow \mathbb{G}_{m, k}, x \mapsto x^{2}$, являются покрытиями (в частности, $\mathscr{F}\left(\mathbb{A}_{X}^{1}\right) \rightarrow \mathscr{F}\left(\mathbb{G}_{m, X}\right)$ инъективно для любого $X \in \mathscr{V}_{k}$ и любого пучка на $\left.\mathscr{V}_{k}\right)$. 
ПРЕДЛОЖЕНИЕ 3.4.10 [15]. Любой пучок $\mathscr{F}$ на $\mathscr{V}_{k}$ со значениями в $\mathscr{L}$ является $\mathbb{A}^{1}$-инвариантным.

Для доказательства нужно заметить, что фактор $\mathbb{A}^{1} \times \mathbb{A}^{1} \backslash \Delta_{\mathbb{A}^{1}}$ по перестановке $\sigma$ двух множителей $\mathbb{A}^{1}$ "более или менее" совпадает с $\mathbb{A}^{1} \times \mathbb{A}^{1}$ и, значит, $\mathscr{F}\left(U \times \mathbb{A}^{1} \times \mathbb{A}^{1}\right)$ вкладывается в $\mathfrak{S}_{2}$-инвариантную часть $\mathscr{F}\left(U \times \mathbb{A}^{1} \times \mathbb{A}^{1}\right)$, т. е. $\sigma$ действует тривиально. Поскольку $U \times \mathbb{A}^{1} \rightarrow U-$ покрытие, $\operatorname{pr}_{1}=\mathrm{pr}_{2} \circ \sigma$ и $\mathscr{F}(U)$ - уравнитель инъекций $\operatorname{pr}_{1}^{*}, \operatorname{pr}_{2}^{*}: \mathscr{F}\left(U \times \mathbb{A}^{1}\right) \rightrightarrows \mathscr{F}\left(U \times \mathbb{A}^{1} \times \mathbb{A}^{1}\right)$, индуцированных проекциями, получаем, что $\mathscr{F}(U) \rightarrow \mathscr{F}\left(U \times \mathbb{A}^{1}\right)$ - изоморфизм.

3.5. Представления, коиндуцированные с открытых подгрупп. В этом пункте мы приведем пример (в предложении 3.5.3) пары существенно различных открытых компактных подгрупп $U$ и $U^{\prime}$ в $G$ таких, что имеются вложения $E$-представлений $E[G / U] \hookrightarrow E\left[G / U^{\prime}\right]$ и $E\left[G / U^{\prime}\right] \hookrightarrow E[G / U]$ группы $G$. Из этого следует, что неприводимые подфакторы $E[G / U]$ и $E\left[G / U^{\prime}\right]-$ одни и те же. В этом примере примитивные мотивы максимального уровня моделей полей $F^{U}$ и $F^{U^{\prime}}$ совпадают (и зануляются). Однако, как показывает пример предложения 3.5.4, совпадение неприводимых подфакторов - явление довольно общего характера.

Но сделаем сначала несколько общих замечаний.

ЗАмЕчАнИЕ 3.5.1. (i) Если $U \subset U^{\prime} \subset H$ - подгруппы и индекс $U$ в $U^{\prime}$ конечен, то имеется естественное вложение $E\left[H / U^{\prime}\right] \hookrightarrow E[H / U]$, заданное формулой $[u] \mapsto \sum_{h \in H / U, h U^{\prime}=u U^{\prime}}[h]$.

(ii) Представления группы $G / G^{\circ}$. Пусть $E$ - произвольное поле и $U-$ компактная открытая подгруппа в $G$. Тогда имеется сюръекция $E$-представления $E[G / U]$ группы $G$ на любое неприводимое $E$-представление группы $G$, пропускающееся через фактор $G / G^{\circ}$.

ПРЕДЛОЖЕНИЕ 3.5.2. Пусть $1 \leqslant n \leqslant \infty, F^{\prime} \mid k$ - алгебраически замкнутое расширение в F конечной степени трансцендентности и $\varphi$ - гладкое неприводимое E-представление группь $G_{F^{\prime} \mid k}$. Пусть $W-$ любой неприводимый фактор (циклического, порожденного любым ненулевым элементом из $\left.V^{G_{F \mid F^{\prime}}}=[\mathrm{id}] \otimes \varphi\right)$ представления $V:=E\left[G / G_{F \mid F^{\prime}}\right] \otimes_{E\left[G_{F^{\prime} \mid k}\right]} \varphi$. Тогда в $W^{G_{F \mid F^{\prime}}}$ имеется не более $\max (|k|,|E|)$ неприводимых подпредставлений группы $G_{F^{\prime} \mid k}$, одно из которьх - $\varphi$.

Группа $G$ имеет не менее $|k|$ допустимых неприводимых представлений. Если $k$ и Е счетны, то гладких неприводимых представлений группы $G$ ровHo $2^{|\mathbb{N}|}$.

ДокАЗАТЕЛЬство. Заметим, что только одномерных над $E$ представлений группы $G_{F^{\prime} \mid k}$, которые пропускаются через модуль группы $G_{F^{\prime} \mid k}$, имеется ровно $\left|\operatorname{Hom}\left(\mathbb{Q}_{+}^{\times}, E^{\times}\right)\right|=\max \left(2^{|\mathbb{N}|},|E|\right)$ штук. Для каждого $\varphi$ выберем $W=: W_{\varphi}$. Скажем, что $\varphi \sim \psi$, если $W_{\varphi} \cong W_{\psi}$. Поскольку $\left|W^{G_{F \mid F^{\prime}}}\right| \leqslant|W|=\max (|k|,|E|)$, классы эквивалентности имеют мощность, не превосходящую $\max (|k|,|E|)$. Поэтому $|\{\varphi\} / \sim| \geqslant \max \left(2^{|\mathbb{N}|},|E|\right)$, если это $>\max (|k|,|E|)$, т. е. если $2^{|\mathbb{N}|}>|k|$ и $2^{|\mathbb{N}|}>|E|$. В любом случае имеется $|k|$ гладких неприводимых представлений группы $G$ вида $(A(F) / A(k)) \otimes E$, где $A$ - эллиптическая кривая над $k$ без комплексного умножения. 
(iii) Подкрутки одномерными представлениями. Пусть $n<\infty$ и $\varphi$ - гомоморфизм из $G / G^{\circ}$ в $\mathbb{Q}^{\times}$. Рассмотрим $E[G / U](\varphi)$ как то же $E$-векторное пространство, что и $E[G / U]$, но с действием $G$ по формуле $[\sigma] \stackrel{\tau}{\longmapsto} \varphi(\tau) \cdot[\tau \sigma]$. Тогда $\lambda_{\varphi}([\sigma]):=\varphi(\sigma) \cdot[\sigma]$ задает изоморфизм представлений $E[G / U] \stackrel{\lambda_{\varphi}}{\longrightarrow} E[G / U](\varphi)$ группы $G$.

Из этого следует, что для любого неприводимого $E$-представления $W$ группы $G$ кратности $W$ и $W(\varphi)$ в $E[G / U]$ совпадают. Однако, скорее всего, эти кратности бесконечны.

Например, пусть $L$ - расширение $k$ конечного типа и степени трансцендентности $q$ в $F$. Тогда, по крайней мере если выполняются некоторые гипотезы, любой мотивный $G$-модуль уровня $<q$ является подфактором $\mathbb{Q}\left[G / G_{F \mid L}\right]$ бесконечной кратности. Чтобы это увидеть, зафиксируем базис трансцендентности $x_{1}, \ldots, x_{q}$ поля $L$ над $k$. Тогда имеется сюръекция $\mathbb{Q}\left[G / G_{F \mid L}\right] \rightarrow \Omega_{F \mid k}^{s}$, заданная формулой [1] $\mapsto x_{s+1} d x_{1} \wedge \cdots \wedge d x_{s}$ для любого $s<q$. Любой мотивный $G$-модуль уровня $s$ является подмодулем $\Omega_{F \mid k}^{s}$ бесконечной кратности.

Чисто трансцендентные расширения квадратичных расширений.

ПреДЛОЖЕНИЕ 3.5.3 [7; следствие 7.3]. Пусть $L^{\prime \prime} \subset F-$ подполе, конечно порожденное над $k, u F \neq \overline{L^{\prime \prime}}$. Для некоторого $u \in \sqrt{\left(L^{\prime \prime}\right)^{\times}} \backslash\left(L^{\prime \prime}\right)^{\times}$и некоторого $t \in F$, трансцендентного над $L^{\prime \prime}$, положим $L=L^{\prime \prime}(u, T)$, где $T=(2 t-u)^{2}$, u $L^{\prime}=L^{\prime \prime}(t)$. Тогда для $U=G_{F \mid L} u U^{\prime}=G_{F \mid L^{\prime}}$ существуют вложения $E\left[G / U^{\prime}\right] \hookrightarrow E[G / U]$ u $E[G / U] \hookrightarrow E\left[G / U^{\prime}\right]$.

Это вытекает из следующего комбинаторного утверждения [7; лемма 7.2].

Пусть $H$ - группа, а $U$ и $U^{\prime}$ - такие подгруппь $H$, что индекс $U \cap U^{\prime}$ в $U$ равен двум: $U=\left(U \cap U^{\prime}\right) \cup \sigma\left(U \cap U^{\prime}\right)$. Предположим, что $\tau_{1} \cdots \tau_{N} \neq 1$ для любого иелого $N \geqslant 1$ и любого набора $\tau_{1}, \ldots, \tau_{N} \in U^{\prime} \sigma \backslash U$. Тогда морбизм E-представлений $E[H / U] \stackrel{[\xi] \mapsto[\xi \sigma]+[\xi]}{\longrightarrow} E\left[H / U^{\prime}\right]$ группы $H$ ингективен.

ПрЕДЛОжЕНИЕ 3.5.4 [7; предложение 7.4]. Зафиксируем нечетное иелое $m \geqslant 1$, и пусть $m-1 \leqslant n \leqslant \infty$. Зафиксируем набор $x_{1}, \ldots, x_{m}$ элементов $F$ с единственным соотношением $\sum_{j=1}^{m} x_{j}^{d}=1$ над $k$, где $d \in\{m+1, m+2\}$. Положим $L^{\prime \prime}=k\left(x_{1}, \ldots, x_{m}\right)$ u $L=\left(L^{\prime \prime}\right)^{\left\langle e_{1} e_{2}^{2} \cdots e_{m}^{m}\right\rangle}$, где $e_{i} x_{j}=\zeta^{\delta_{i j}} \cdot x_{j} \partial л я$ примитивного $d$-го корня из единицы $\zeta$. Пусть $L^{\prime}-$ максимальное чисто трансцендентное расширение $k$ в $L$. Тогда если $U=G_{F \mid L}$ u $U^{\prime}=G_{F \mid L^{\prime}}$, то $E$-представления $E[G / U]$ и $E\left[G / U^{\prime}\right]$ группы $G$ имеют одни и те же неприводимые подфакторы.

ПРЕДЛОЖЕНИЕ 3.5.5. Пусть $g_{1}, \ldots, g_{N}$ - рациональные инволюиии $k$-многообразия $X$, которые порождают бесконечную группу. Тогда естественное отображсние E-представлений $r: E[\{k(X) \stackrel{/ k}{\hookrightarrow} F\}] \rightarrow \bigoplus_{j=1}^{N} E\left[\left\{k(X)^{\left\langle g_{j}\right\rangle} \stackrel{/ k}{\hookrightarrow} F\right\}\right]$ группь $G$ ингективно.

ДокАЗАТЕЛЬСтво. Если ненулевой 0-цикл $\alpha$ лежит в ядре $r$ и $P$ - точка в носителе $\alpha$, то носитель $\alpha$ содержит $\left\langle g_{1}, \ldots, g_{N}\right\rangle$-орбиту точки $P$. Поскольку эта орбита бесконечна, а носитель $\alpha$ конечен, получаем противоречие, т. е. $\alpha=0$. 
ПримеР 3.5.6. (i) Пусть $X$ - алгебраическая $k$-группа, $g_{1}: x \mapsto x^{-1}$ и $g_{2}$ : $x \mapsto h \cdot x^{-1}$, где $h \in X(k)$ - точка бесконечного порядка. Тогда $E$-представления $E[\{k(X) \stackrel{/ k}{\hookrightarrow} F\}]$ и $E[\{k(K(X)) \stackrel{/ k}{\hookrightarrow} F\}]$ группы $G$ имеют одни и те же неприводимые подфакторы, где $K(X)$ - фактор $X$ по инволюции $g_{1}$ (многообразие Куммера).

(ii) Если $Y_{j}$ - двулистные в общей точке накрытия проективных пространств над $k$, из которых хотя бы одно, например $Y_{1},-$ кривая рода $\leqslant 1$, то имеются вложения

$$
\begin{aligned}
E\left[\left\{k\left(\prod_{j=1}^{N} Y_{j}\right) \stackrel{/ k}{\hookrightarrow} F\right\}\right] & \hookrightarrow \bigoplus_{i=1}^{N} E\left[\left\{k\left(\prod_{1 \leqslant j \leqslant N, j \neq i} Y_{j}\right)\left(\mathbb{P}^{d_{i}}\right) \stackrel{/ k}{\hookrightarrow} F\right\}\right]^{1+\delta_{1 i}} \\
& \hookrightarrow E\left[\left\{k\left(\mathbb{P}^{d}\right) \stackrel{/ k}{\hookrightarrow} F\right\}\right]^{N+1}
\end{aligned}
$$

где $d_{i}=\operatorname{dim} Y_{i}$ и $d=\sum_{j=1}^{N} d_{j}$.

\section{4. Гомотопически инвариантные представления группы $G$}

В этом разделе продолжено описание абелевой категории $\mathscr{I}_{G}$ (см. п. 1.1).

Категория $\mathscr{I}_{G}$ замкнута относительно перехода к подфакторам в $\mathscr{S} m_{G}$ (лемма 4.1.1). При $n=\infty$ это - подкатегория Серра в $\mathscr{S} m_{G}\left(\right.$ теорема 1.1.10,1)), ${ }^{4}$ замкнутая относительно внутреннего функтора $\mathscr{H} o m$ на $\mathscr{S} m_{G}$ (предложение 4.1.14). Из леммы 3.4.1 и предложения 3.4.10 следует, что $\mathscr{I}_{G}(E)$ содержит категорию $\mathscr{A} d m_{G}(E)$ допустимых представлений $G$ (теорема $\left.1.1 .10,2\right)$ ), если $n=\infty$. Прямое (но такое же по сути) доказательство приводится в [7; предложение 6.4].

Функтор включения $\mathscr{I}_{G} \hookrightarrow \mathscr{S}_{G}$ допускает левый сопряженный

$$
\mathscr{I}=\left(\lim _{L} C_{L}\right) \otimes_{\mathbb{D}_{\mathbb{Q}}}: \mathscr{S} m_{G} \rightarrow \mathscr{I}_{G},
$$

см. п. 4.6, так что любой морфизм из $W \in \mathscr{S} m_{G}$ в объект $\mathscr{I}_{G}$ пропускается через $\mathscr{I} W \in \mathscr{I}_{G}$ (предложение 4.1.3).

В случае $n=\infty$ в категории $\mathscr{S} m_{G}$ нет ненулевых проективных объектов (замечание на с. 119). В отличие от категории $\mathscr{S} m_{G}$, в категории $\mathscr{I}_{G}$ достаточно много проективных объектов (теорема $1.1 .10,4)$ ). А именно, объекты $C_{L}:=\mathscr{I} \mathbb{Q}[\{L \stackrel{/ k}{\hookrightarrow} F\}]$ для всех расширений полей конечного типа $L \mid k$ задают систему проективных образующих категории $\mathscr{I}_{G}$. Пучок $X \mapsto C_{k(X)}$ на $\mathfrak{D} m_{k}$ со значениями в $\mathscr{I}_{G}^{\mathrm{op}}$ (следствие 3.4.5) $\mathbb{A}^{1}$-инвариантен (лемма 4.1.10).

Для любого гладкого неприводимого собственного многообразия $X$ над $k$ имеется естественная сюръекция $C_{k(X)} \rightarrow C H_{0}\left(X \times_{k} F\right)_{\mathbb{Q}}$. Первая часть гипотезы 1.1.11 утверждает, что это - изоморфизм при $n=\infty$. Если $X-$ кривая, то это проверено в предложении 4.1.11. С этой гипотезой связано существование тензорной структуры на $\mathscr{I}_{G}$, см. замечание 1.1 .12 , (iii).

\footnotetext{
${ }^{4}$ При $n<\infty$ имеются нетривиальные расширения $\mathbb{Q}$ при помощи $\mathbb{Q}$, т. е. категория $\mathscr{I}_{G}$ не замкнута относительно расширений в $\mathscr{S} m_{G}$.
} 
По всей видимости, категорию смешанных мотивов можно связать с некоторой полной подкатегорией в категории гладких $G$-модулей, объекты которой имеют "мотивные" неприводимые подфакторы. Так, по аналогии с теорией Ходжа, предположено (гипотеза 4.1.7), что присоединенные факторы фильтрации уровня $N$ • полупросты для любого объекта $\mathscr{I}_{G}$. Из этого нетрудно вывести (следствие 4.1.8), что фильтрация уровня $N$ • строго совместима с морфизмами в $\mathscr{I}_{G}$. В частности, расширения $G$-модулей из $\mathscr{I}_{G}$ меньшего уровня неприводимыми $G$-модулями из $\mathscr{I}_{G}$ большего уровня (канонически) расщеплены. В свою очередь, гипотеза 4.1.7 следует из первой части гипотезы 1.1.11 и "мотивных" гипотез, см. замечание 1.1.12, (ii).

Хотелось бы расширить категорию $\mathscr{I}_{G}$, чтобы рассматривать такие $G$-модули, как $F^{\times} / k^{\times}$, и продолжить фильтрацию $N \bullet$ на $\mathscr{I}_{G}$ до некоторой “весовой фильтрации" так, чтобы свойство строгой совместимости с морфизмами сохранилось.

Заметим, что фильтрация $N_{\bullet}$ на гладких $G$-модулях не является строго совместимой с морфизмами. Например, любой неприводимый допустимый $G$-модуль уровня 1 (соответствующий некоторому абелевому многообразию) допускает нетривиальное расширение неприводимым $G$-модулем $F^{\times} / k^{\times}$(который также уровня 1), см. контрпример после предложения 1.1.18.

Как правило, вес неприводимого объекта $W_{1}$ больше веса неприводимого объекта $W_{2}$, если $\operatorname{Ext}^{1}\left(W_{1}, W_{2}\right) \neq 0$, и, следовательно, вес $(\mathbb{Q})<\operatorname{веc}\left(F^{\times} / k^{\times}\right)<$ $\operatorname{веc}(A(F) / A(k))$ для любого абелева многообразия $A$ над $k$. Если веса согласованы с мотивными весами и $A(F) / A(k)$ соответствует мотиву $H_{1}(A)$ веса 1 , то вес представления $F^{\times} / k^{\times}$оказывается натуральным числом, меньшим единицы. Вариантом решения этой проблемы могла бы стать градуировка ранга $>1$.

Эта бо́льшая категория должна допускать функтор двойственности, которого нет в случае категории $\mathscr{I}_{G}$.

Резюмируем следующим образом основные результаты п. 4.3, помимо упомянутых в п. 1.1 .

Теорема 4.0.1. 1) Для любых $1 \leqslant n \leqslant \infty u q \geqslant 0$ имеется функтор $\mathfrak{B}^{q}$ :

$$
\left\{\begin{array}{c}
\text { чистые примитивные } \\
q \text {-мотивы над } k
\end{array}\right\} \stackrel{\mathfrak{B}^{q}}{\longrightarrow}\left\{\begin{array}{c}
\text { полупростые допустимые } G \text {-модули } \\
\text { конечного типа и уровня } q
\end{array}\right\} \text {, }
$$

вполне строгий при $q \leqslant n$. (Под уровнем $G$-модуля $W$ понимается такое $q$, что $N_{q} W=W$ и $N_{q-1} W=0$ для фильтрации $N_{\bullet}$, определенной после теоремъь 1.1.10.)

2) Если $n<\infty$, то на $G$-модуле $\mathfrak{B}^{n}(M)$ имеется билинейная симметрическая невырожденная $G$-эквивариантная форма со значениями в ориентированном $G$-модуле $\mathbb{Q}(\chi)$ степени 1 , где $M=\left(X, \Delta_{k(X)}\right)$ - максимальный примитивный $n$-подмотив мотива $\left(X, \Delta_{X}\right) u \operatorname{dim} X=n$.

Эта форма определена, если для $(n-1)$-циклов на $2 n$-мерных комплекснъх многообразиях численная эквивалентность совпадает с гомологической (например, при $n \leqslant 2$ ) и, следовательно, $\mathfrak{B}^{n}$ пропускается через подкатегорию "поляризуемых" G-модулей (т.е. допускающих положительную форму как выше). 
Эта теорема прямо вытекает из следствия 3.1.7 и предложений 4.3.2, 4.3.12 и 4.3.14. Грубо говоря, функтор $\mathfrak{B}^{q}$ определен как пространство 0-циклов над $F$ по модулю “численной эквивалентности над $k$ ". Подробности см. в п. 4.3, c. 141, где показано, что он пропредставим. Из предложения 4.3 .9 следует, что $\mathfrak{B}^{q}((X, \pi))$ зависит только от класса бирациональной эквивалентности $X$. Более того, из предложения 3.3 .2 следует, что композиция функтора $\mathfrak{B}^{1}$ с забывающим функтором в категорию $G^{\circ}$-модулей также вполне строга, и функтор $\mathfrak{B}^{1}$ из теоремы 4.0.1, 1) - эквивалентность категорий при $n=\infty$, см. п. 4.1.

Гипотеза 1.1.7 допускает следующую форму, удобную для проверки в частных случаях.

ГиПотезА 4.0.2. Для любого $q \geqslant 0$ функтор $\mathfrak{B}^{q}$ задает эквивалентность категорий при $n=\infty$.

Можно показать, что если $U \neq 0$ - фактор $F^{\times}$, то функтор $U \otimes: \mathscr{I}_{G} \rightarrow$ $\mathscr{S} m_{G}-$ вполне строгий. Поэтому помимо $\mathbb{B} \bullet$ существуют другие вполне строгие функторы из категории чистых мотивов в категорию гладких градуированных представлений $G$, см. также лемму 1.2.6. Однако эти функторы не сохраняют неприводимость. ${ }^{5}$

Имеются указания на то, что категория примитивных $n$-мотивов "недалека" от категории поляризуемых (в смысле теоремы 4.0.1, 2)) $G$-модулей (по крайней мере, если $n \leqslant 2$ ). Так, обращение в нуль неподвижных (относительно компактной подгруппы) подпространств в поляризуемых $G$-представлениях $G_{F \mid L(x)}$ (здесь $L \mid k$ - подрасширение в $F$ и $x$ - такой элемент поля $F$, трансцендентный над $L$, что $F=\overline{L(x)})$ соответствует тривиальности примитивных $n$-подмотивов мотива $\left(Y \times \mathbb{P}^{1}, \pi\right)$, где $\operatorname{dim} Y<n$. Однако необходимо наложить дополнительные условия, поскольку из предложения 3.3 .2 и полной строгости функтора $\mathfrak{B}^{1}$ нетрудно вывести, что для любого $G$-модуля $W$ существует не более одного характера $\psi$ такого, что $W(\psi) \cong \mathfrak{B}^{1}(M)$ для чистого 1-мотива $M$ (см. [7; следствие 4.5]), а подкрутки поляризуемых $G$-модулей характерами группы $G$ порядка 2 также поляризуемы.

Возможные связи со смешанными мотивами. Несколько теорий когомологий алгебраических многообразий связаны изоморфизмами сравнения и ведут себя параллельным образом. Это подвело А. Гротендика, П. Делиня, А.А. Бейлинсона и др. к гипотезе о существовании универсальной теории когомологий - со значениями в абелевой категории смешанных мотивов и о тождествах между группами расширений между этими когомологическими объектами и $K$-группами. Ссылками для этого круга идей являются, например, [8], [35].

\footnotetext{
${ }^{5} W \otimes U$ неприводимо, только если $U$ неприводимо и $W \neq U$. Поэтому, если $U \neq 0-$ фактор $A(F)$ для некоторой коммутативной $k$-группы $A$, то можно считать, что $A$ - простая $k$-группа и не абелево многообразие, т. е. или $\mathbb{G}_{m}$, или $\mathbb{G}_{a}$, и что $U=A(F) / A(k)$. Если $W=E(F) / E(k)$ для некоторой эллиптической кривой $E$ над $k$, то морфизм $W \otimes U \stackrel{\omega \wedge \eta}{\longrightarrow} \Omega_{F \mid k}^{2}$, где $\omega$ - ненулевая регулярная 1-форма на $E$, а $\eta$ - ненулевая инвариантная 1-форма на $A,-$ ненулевой и не инъективен, т. е. $W \otimes U$ приводимо.
} 
Для гладких проективных многообразий эта теория задается мотивами Гротендика, но только при условии, что численная эквивалентность совпадает с гомологической.

В. А. Воеводский, М. Левин и М. Ханамура (см. [36]-[38]) определили триангулированные категории, которые, как ожидается, должны быть эквивалентны производной категории смешанных мотивов. Основная трудность состоит в построении $t$-структуры, сердцевиной которой была бы искомая абелева категория смешанных мотивов. Это можно было бы сделать, если бы были доказаны “стандартные” гипотезы (в том числе гипотезы Бейлинсона), см. [10].

Другой подход, Делиня и Яннсена (см. [39]), состоит в рассмотрении согласованных наборов "реализаций”. Здесь трудности связаны с недоказанностью гипотез Ходжа и Тэйта.

Как упоминалось в п. 1.1, с данной теорией когомологий $H^{*}$ можно связать $G$-модуль $H_{c}^{*}(F):=\lim H^{*}(Y) / N^{1} H^{*}(Y)$, где $Y$ пробегает все гладкие собственные неприводимые многообразия над $k$ с полями функций, вложенными в $F$, и $N^{\bullet}$ - фильтрация коуровня. Ясно, что $H_{c}^{*}(F) \in \mathscr{I}_{G}$ и $H_{c}^{*}(F) \in \mathscr{A} d m_{G}(E)$, если $H^{*}(k)$ - конечномерное $E$-векторное пространство. Тогда (в предположении, что численная эквивалентность совпадает с гомологической) $H_{c}^{*}(F)$ - полупростой $G$-модуль, допускающий разложение $H_{c}^{*}(F) \cong \bigoplus_{M} H^{*}(M) \otimes_{\operatorname{End}(M)}$ $\mathbb{B}(M)$, где $M$ пробегает классы изоморфизма неприводимых примитивных мотивов (как в предложении 1.1.18). Отсюда следует, что, в обозначениях п. 4.3, $H^{*}(N) \cong \bigoplus_{i} \operatorname{Hom}_{G}\left(\mathbb{B}^{[i]}(N), H_{c}^{*}(F)\right)$ для любого мотива $N$. Таким образом, функтор реализации (на категории чистых мотивов над $k$ ), соответствующий теории $H^{*}$, разлагается в композицию функтора $\mathbb{B}^{\bullet}$ и (контравариантного) функтора $\operatorname{Hom}_{G}\left(-, H_{c}^{*}(F)\right)$ (на категории $G$-модулей).

\section{1. Категория $\mathscr{I}_{G}$, фильтрация $N_{\bullet}$ и дифференциальные формы.}

Лемма 4.1.1 [7; лемма 6.6]. Функтор $H^{0}\left(G_{F \mid L},-\right): \mathscr{I}_{G} \rightarrow \mathscr{V}_{\text {ect }} t_{\mathbb{Q}}$ точен для любого расширения $L$ поля $k$ в $F$. Подкатегория $\mathscr{I}_{G}$ замкнута относительно взятия подфакторов в $\mathscr{S} m_{G}$.

Для каждого целого $q \geqslant 0$ пусть $\mathscr{I}_{G}^{q}-$ полная подкатегория в $\mathscr{I}_{G}$ с такими обгектами $W$, что $W^{G_{F \mid F^{\prime}}}=0$ для любого алгебраически замкнутого $F^{\prime}$ $c \operatorname{deg} \operatorname{tr}\left(F^{\prime} \mid k\right)=q-1$. Тогда $\left\{\mathscr{I}_{G}^{q}\right\}_{q \geqslant 0}-$ убъвающая фильтраиия категории $\mathscr{I}_{G}$ подкатегориями Серра. ${ }^{6}$

Отсюда следует, что $\mathscr{A} d m_{G}(E)$ - (абелева) подкатегория Серра в $\mathscr{S} m_{G}(E)$ [7; следствие 6.5].

Лемма 4.1.2 [7; лемма 6.7]. Если $F^{\prime} \mid k$ - расширение в $F$ бесконечной степени трансцендентности, то функтор $H^{0}\left(G_{F \mid \overline{F^{\prime}}},-\right)$ из $\mathscr{S} m_{G}$ в $\mathscr{S} m_{G_{\overline{F^{\prime}} \mid k}}$ задает эквивалентность категорий (индуцирующую эквивалентность $\mathscr{I}_{G}$ и $\left.\mathscr{I}_{G_{\overline{F^{\prime}} \mid k}}\right)$. Функтор $H^{0}\left(G_{F \mid K},-\right)$ из $\mathscr{S}_{G_{G}}$ в $\mathscr{V}$ есt $t_{\mathbb{Q}}$ точен тогда и только тогда, когда $\operatorname{deg} \operatorname{tr}(K \mid k)=\operatorname{deg} \operatorname{tr}(F \mid k)(\leqslant \infty)$.

Доказательство использует изоморфизм полей $F \stackrel{\sim}{\longrightarrow} \overline{F^{\prime}}$, тождественный на $k$.

${ }^{6}$ Полная подкатегория абелевой категории $\mathscr{B}$ называется подкатегорией Серра, если она замкнута относительно взятия подфакторов и расширений в $\mathscr{B}$. 
Функтор $\mathscr{I}$. Фильтрация уровня $N$ • на $G$-модуле $M$ была определена на c. 93. Эквивалентно, $N_{j} M$ - минимальное подпредставление группы $G$ в $M$, содержащее $M^{G_{F \mid F_{j}}}$ для некоторого алгебраически замкнутого $F_{j} \subseteq F$. Ясно, что $N_{\bullet}$ - функториальная (ограничение на $N_{j} M$ каждого $G$-гомоморфизма $M \rightarrow M^{\prime}$ пропускается через $\left.N_{j} M^{\prime}\right)$, неотрицательная, возрастающая $\left(N_{j} M \subseteq\right.$ $\left.N_{j+1} M\right)$, мультипликативная (по отношению к тензорным произведениям: $\left.N_{i+j}\left(M_{1} \otimes M_{2}\right) \supseteq N_{i} M_{1} \otimes N_{j} M_{2}\right)$ фильтрация, исчерпывающая на гладких представлениях ( $M=\bigcup_{j \geqslant 0} N_{j} M$, если $M$ - гладкое).

ПРЕДЛОЖЕНИЕ 4.1.3 [7; предложение 6.8]. Для любого целого $q \geqslant 0$ любой объект $W \in \mathscr{S} m_{G}$ допускает такой фактор $\mathscr{I}^{q} W \in \mathscr{I}_{G}^{q}$, что любой $G$-гомоморфизм из $W$ в любой обгект $\mathscr{I}_{G}^{q}$ пропускается через $\mathscr{I}^{q} W$. Функтор $\mathscr{S} m_{G} \stackrel{\mathscr{I}^{q}}{\longrightarrow} \mathscr{I}_{G}^{q}$, заданный $W \mapsto \mathscr{I}^{q} W$, точен справа и

$$
\mathscr{I}^{q} W=\mathscr{I} W / N_{q-1} \mathscr{I} W
$$

Существование функторов $\mathscr{I}^{q}$ можно вывести из общекатегорных фактов, см. [40; §5.8], однако в [7] они строятся "явно", что позволяет увидеть следующую связь образующих категории $\mathscr{I}_{G}$ с группами Чжоу 0-циклов.

ПРеДлОЖЕНИЕ 4.1 .4 [7; предложение 6.17]. Если $n=\infty$, то для любого неприводимого многообразия $X$ над $k$ ядро проекции $\mathbb{Q}[\{k(X) \stackrel{/ k}{\hookrightarrow} F\}] \rightarrow C_{k(X)}{ }^{-}$ сумма по всем кривым $y \in\left(k(X) \otimes_{k} F\right)_{1}$ подпространств, натянутых на те линейные комбинации общих (по отношению $к$ некоторому полю определения кривых у) F-точек кривых $\overline{\{y\}}$, которые линейно эквивалентны нулю на любой компактификации $\overline{\{y\}}$.

ПримеР 4.1.5. Пусть $A$ - одномерная групповая схема над $k, m \geqslant 1$ - целое и $W=N_{q} W$ - гладкое представление группы $G$, где $q \leqslant n-1$. Тогда $\mathscr{I}\left(S^{m} A(F) \otimes W\right)=0$ в следующих трех случаях: или если $m$ четно, или если $A=\mathbb{G}_{a}$, или если $A=\mathbb{G}_{m}$. В частности, естественная проекция $A(F)_{\mathbb{Q}}^{\otimes N} \rightarrow$ $\bigwedge_{\operatorname{End}_{\mathbb{Q}} A}^{N} A(F)_{\mathbb{Q}}$ индуцирует изоморфизм $\mathscr{I}\left(A(F)_{\mathbb{Q}}^{\otimes N}\right) \stackrel{\sim}{\longrightarrow} \mathscr{I}\left(\bigwedge_{\operatorname{End}_{\mathbb{Q}} A}^{N} A(F)_{\mathbb{Q}}\right)$, если $n \geqslant N-1$. (Если End $A=\mathbb{Z}$, то это проверено в [7; с. 204]; общий случай аналогичен.)

ЗАмечАниЕ 4.1.6. Функтор $\mathscr{I}$ не является точным слева. Например, он преобразует вложение $k \hookrightarrow F$ в $k \rightarrow 0$.

ГиПотеЗА 4.1.7. Если $n=\infty$, то для любого $j \geqslant 0$ и любого обгекта $W$ категории $\mathscr{I}_{G}$ представление $\operatorname{~gr}_{j}^{N} W$ группљ $G$ полупросто.

Это очевидно в случае $j=0$ и легко выводится из следствия 4.1 .12 в случае $j=1$.

СлЕДСТвИЕ 4.1.8. Предположим, что $n=\infty$ и гипотеза 4.1 .7 верна для любого $0 \leqslant j \leqslant q-1$. Пусть $L \mid k$ - расширение полей конечного типа и $\operatorname{deg} \operatorname{tr}(L \mid k)<q$. Тогда:

- функтор $\mathscr{I}^{q}$ точен на $\mathscr{I}_{G}$ (что эквивалентно строгой совместимости фильтрации $N_{0} \subseteq \cdots \subseteq N_{q-1}$ с морфизмами в $\left.\mathscr{I}_{G}\right)$; 
- алгебра $A=C H^{s}\left(\operatorname{Spec}\left(L \otimes_{k} L\right)\right)$, где $s=\operatorname{deg} \operatorname{tr}(L \mid k)$, полупроста и длина ее конечна ${ }^{7}$.

Первая часть этого следствия доказана в [7; следствие 6.10], а вторая очевидна из формулы $A=\operatorname{End}_{G}(W)$, где $W:=C H^{s}\left(\mathbf{S p e c}\left(L \otimes_{k} F\right)\right)=\operatorname{gr}_{s}^{N} W$ полупросто и циклично.

ЗАмЕчАниЕ 4.1.9. Пример морфизма гладких $G$-модулей, не строго совместимого с фильтрацией $N_{\bullet}$, дает включение $\mathbb{Q}\left[G / G_{F \mid L}\right]^{\circ} \hookrightarrow \mathbb{Q}\left[G / G_{F \mid L}\right]$, поскольку $N_{\operatorname{deg} \operatorname{tr}(L \mid k)} \mathbb{Q}\left[G / G_{F \mid L}\right]^{\circ}$ совпадает с

$$
\left\{\sum_{[\sigma] \in G / G_{F \mid L}} a_{\sigma}[\sigma] \mid \sum_{\sigma(L) \subset \overline{F^{\prime}}} a_{\sigma}=0 \text { для любого } F^{\prime} \mathrm{c} \operatorname{degtr}\left(F^{\prime} \mid k\right)=\operatorname{degtr}(L \mid k)\right\}
$$

что отличается от $\mathbb{Q}\left[G / G_{F \mid L}\right]^{\circ}$, но $\mathbb{Q}\left[G / G_{F \mid L}\right]=N_{\operatorname{deg} \operatorname{tr}(L \mid k)} \mathbb{Q}\left[G / G_{F \mid L}\right]$.

Лемма 4.1.10 [7; лемма 6.12]. Для любого $1 \leqslant n \leqslant \infty$, любого подполя $L_{1} \subset F$ конечного типа над $k$ и любого унирационального расширения $L_{2}$ поля $L_{1}$ в F конечного типа имеется естественный изоморбизм $C_{L_{2}} \stackrel{\sim}{\longrightarrow} C_{L_{1}}$.

Объекты категории $\mathscr{I}_{G}$ уровня 1 . Для любого $W \in \mathscr{S} m_{G}$ имеется сюръекция $\bigoplus_{e \in W^{G} F \mid F^{\prime}}\langle e\rangle_{G} \rightarrow N_{1} W$, где $F^{\prime} \mid k$ - алгебраически замкнутое расширение в $F$ с $\operatorname{deg} \operatorname{tr}\left(F^{\prime} \mid k\right)=1$. Это значит, что для того, чтобы описать объекты $\mathscr{I}_{G}$ уровня 1 , достаточно разобраться со случаем $W=\langle e\rangle_{G}$, где $\mathrm{Stab}_{e} \supseteq G_{F \mid L}$ с $L \cong k(X)$ для гладкой собственной кривой $X$ над $k$ рода $g \geqslant 0$. Тогда $W$ доминируется представлением $C_{L}$. Пусть $\operatorname{Pic}^{j} X-$ многообразие Пикара классов линейной эквивалентности дивизоров на $X$ степени $j$.

ПРЕДЛОЖЕНИЕ 4.1.11 [7; предложения 6.20, 6.21]. Пусть X- гладкая проективная кривая над $k, k(X)$ - ее поле функций, $Z_{0}^{\mathrm{rat}}\left(k(X) \otimes_{k} F\right)$ - ядро естественной проекиии $\mathbb{Q}[\{k(X) \stackrel{/ k}{\hookrightarrow} F\}] \rightarrow \operatorname{Pic}\left(X_{F}\right)_{\mathbb{Q}} u \mathbb{Q}[\{k(X) \stackrel{/ k}{\hookrightarrow} F\}]^{\circ}-$ группа общих 0-циклов над F нулевой степени. Если $n=\infty$, mо $\mathscr{I} Z_{0}^{\operatorname{rat}}\left(k(X) \otimes_{k} F\right)=0$, $\mathscr{I} \mathbb{Q}[\{k(X) \stackrel{/ k}{\hookrightarrow} F\}]^{\circ}=\operatorname{Pic}^{\circ}\left(X_{F}\right)_{\mathbb{Q}} u C_{k(X)}=\operatorname{Pic}\left(X_{F}\right)_{\mathbb{Q}} \cdot$

Доказательство основано на том, что а) достаточно большие симметрические степени гладкой проективной кривой - это проективные расслоения над ее якобианом, и б) [7; лемма 6.18]: $G$-модуль $Z_{0}^{\text {rat }}\left(k(X) \otimes_{k} F\right)$ порожден элементами $w_{N}=\sum_{j=1}^{N} \sigma_{j}-\sum_{j=1}^{N} \tau_{j}$ для всех $N \gg 0$, где $\left(\sigma_{1}, \ldots, \sigma_{N} ; \tau_{1}, \ldots, \tau_{N}\right)-$ общая $F$-точка слоя над нулем морфизма $X^{N} \times_{k} X^{N} \stackrel{p_{N}}{\longrightarrow} \operatorname{Pic}^{\circ} X$, посылающего точку $\left(x_{1}, \ldots, x_{N} ; y_{1}, \ldots, y_{N}\right)$ в класс $\sum_{j=1}^{N}\left(x_{j}-y_{j}\right)$.

СлЕДСТвИЕ 4.1 .12 [7; следствия 6.22, 6.23]. Если $n=\infty$, mo $A(F)_{\mathbb{Q}}-$ nроективный обгект категории $\mathscr{I}_{G}$ для любого абелева $k$-многообразия $A$ и любой обгект $\mathscr{I}_{G}$ уровня 1 является прямой суммой тривиального модуля и фактора прямой суммы модулей $A(F)_{\mathbb{Q}}$ для некоторых $A$ по тривиальному подмодулю.

\footnotetext{
${ }^{7}$ Используя следствие 1.1.14, из этого можно было бы вывести, в частности, что алгебра $A$ конечномерна над $\mathbb{Q}$.
} 


\section{Внутренний $\mathscr{H}$ om.}

СлеДСтвиЕ 4.1.13 [7; следствие 6.25]. Для любого расширения $L$ поля $k$ в $F$ c $\operatorname{deg} \operatorname{tr}(F \mid L)=\infty$ и любого неприводимого $k$-многообразия $X$ включение $\mathbb{Q}[\{L(X) \stackrel{/ L}{\hookrightarrow} F\}] \subseteq \mathbb{Q}[\{k(X) \stackrel{/ k}{\hookrightarrow} F\}]$ индуиирует сюргекиию $G_{F \mid L}$-модулей $\mathbb{Q}[\{L(X) \stackrel{/ L}{\hookrightarrow} F\}] \rightarrow C_{k(X)}$.

Теорема $1.1 .10,1)$ и следующее предложение указывают на связь категории $\mathscr{I}_{G}$ с категорией эффективных гомологических мотивов. В п. 4.3 обсуждаются также неэффективные мотивы.

ПРЕДЛОЖЕНИЕ $4.1 .14[7 ; 6.26]$. При $n=\infty$ внутренний функтор $\mathscr{H} о$ нт на

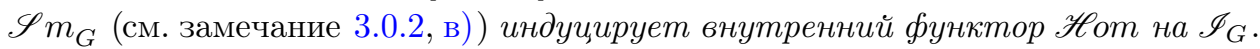
Уровенъ $\mathscr{H} о т\left(W_{1}, W_{2}\right)$ не превосходит $q$, если $W_{1}, W_{2}=N_{q} W_{2} \in \mathscr{I}_{G} u q \leqslant 1$.

ПримеР 4.1.15. Поскольку группа $G$ - коядро $G_{\overline{F(X)} \mid F(X)} \hookrightarrow G_{\{\overline{F(X)}, F\} \mid k(X)}$, она действует на множестве орбит $\{k(Y) \stackrel{/ k}{\hookrightarrow} \overline{F(X)}\} / \operatorname{Gal}(\overline{F(X)} \mid F(X))$. Тогда $\mathscr{H} O m\left(C_{k(X)}, C H_{0}\left(Y_{F}\right)_{\mathbb{Q}}\right)=C H_{0}\left(Y_{F(X)}\right)_{\mathbb{Q}}$.

ЗАмечАниЕ 4.1.16. В отличие от объектов категории $\mathscr{I}_{G}($ в случае $n=\infty)$, для любой топологической группы $H$ существует много гладких представлений $H$ с нетривиальными контрагредиентными представлениями. А именно, $H$-эквивариантное спаривание $\mathbb{Q}[H / U] \otimes \mathbb{Q}[H / U] \rightarrow \mathbb{Q}$, заданное формулами $[\sigma] \otimes[\tau] \mapsto 0$, если $[\sigma] \neq[\tau]$, и $[\sigma] \otimes[\sigma] \mapsto 1$, определяет вложение $\mathbb{Q}[H / U]$ в свое контрагредиентное представление. Здесь $U$ - любая открытая подгруппа $H$.

ПреДлОЖЕНИЕ 4.1 .17 [12; предложение 7.6]. Пусть $W \in \mathscr{I}_{G} u q \geqslant 0-$ иелое. Тогда:

- любой $G$-гомоморфизм $W \stackrel{\varphi}{\rightarrow} \bigotimes_{F}^{q} \Omega_{F \mid k}^{1}$ пропускается через $W \rightarrow \Omega_{F \mid k}^{q} \subseteq$ $\bigotimes_{F}^{q} \Omega_{F \mid k}^{1}$

- для любого гладкого собственного $k$-многообразия $Y$ вложение $k(Y) \stackrel{\iota}{\hookrightarrow} F$ индуцирует ингекцию $\varphi(W) \cap \iota_{*} \Omega_{k(Y) \mid k}^{q} \hookrightarrow \Gamma\left(Y, \Omega_{Y \mid k}^{q}\right)$, и имеются следующ,ие канонические изоморфизмы, функториальные относительно произвольных морфизмов $Y \rightarrow Y^{\prime}:$

$$
\operatorname{Hom}_{G}\left(C_{k(Y)}, \bigotimes_{F}^{\bullet} \Omega_{F \mid k}^{1}\right) \stackrel{\sim}{\longleftarrow} \Gamma\left(Y, \Omega_{Y \mid k}^{\bullet}\right) \stackrel{\sim}{\longrightarrow} \operatorname{Hom}_{G}\left(C H_{0}\left(Y_{F}\right), \bigotimes_{F}^{\bullet} \Omega_{F \mid k}^{1}\right)
$$

НАБРосок ДокАЗАТЕЛЬСтвА. Форма $\omega \in \bigotimes_{F}^{q} \Omega_{F \mid k}^{1}$ интерпретируется как рациональное сечение когерентного пучка $\left.\Omega_{Y^{q} \mid k}^{q}\right|_{\Delta_{Y}}$ на гладком проективном многообразии $Y$ над $k$. Основная идея: если $\omega$ не лежит в сумме образов $\Gamma\left(Y, \Omega_{Y \mid k}^{\bullet}\right)$ для гладких собственных многообразий $Y$ над $k$, то прямой образ $f_{*} \omega=\operatorname{tr}_{/ k\left(\mathbb{P}^{M}\right)}(\omega)$ формы $\omega-$ неподвижный ненулевой элемент $G$-модуля, порожденного $\omega$, для подходящего конечного морфизма $f: Y \rightarrow \mathbb{P}_{k}^{M}$. Чтобы гарантировать, что $f_{*} \omega \neq 0$, используются полюсы. Даже если $\omega$ не имела полюсов, но не лежала в $\Omega_{F \mid k}^{\bullet}$, то полюсы будут у ее прямого образа относительно подходящего конечного разветвленного морфизма. 
4.2. “Формула Кюннета" и тензорная структура. Тензорная структура на $\mathscr{I}_{G}$. Как показывает пример $4.1 .5, \mathscr{I}_{G}$ не замкнута относительно тензорных произведений в $\mathscr{S} m_{G}$. Определим $W_{1} \otimes \mathscr{I} W_{2}$ формулой $\mathscr{I}\left(W_{1} \otimes W_{2}\right)$.

На следующем примере видно, что эта операция не ассоциативна на $\mathscr{S} m_{G}$. Пусть $W_{j}=\mathbb{Q}\left[\left\{k\left(X_{j}\right) \stackrel{/ k}{\hookrightarrow} F\right\}\right]$ для некоторых неприводимых $k$-многообразий $X_{j}$, $1 \leqslant j \leqslant N, N \geqslant 2$. Тогда

$$
W_{1} \otimes \cdots \otimes W_{N}=\bigoplus_{x \in \operatorname{Spec}\left(k\left(X_{1}\right) \otimes_{k} \cdots \otimes_{k} k\left(X_{N}\right)\right)} \mathbb{Q}[\{k(x) \stackrel{/ k}{\hookrightarrow} F\}],
$$

так что $\mathscr{I}\left(W_{1} \otimes \cdots \otimes W_{N}\right)$ изоморфен прямой сумме по всем $x \in \operatorname{Spec}\left(k\left(X_{1}\right) \otimes_{k}\right.$ $\left.\cdots \otimes_{k} k\left(X_{N}\right)\right)$ представлений $C_{k(x)}$. Если $X_{1}=X_{2}=\mathbb{A}_{k}^{1}$, то $\mathscr{I} W_{1}=\mathscr{I} W_{2}=\mathbb{Q}$ и, следовательно, $W_{1} \otimes \mathscr{I}\left(W_{2} \otimes \mathscr{I} \mathbb{Q}\right)=W_{1} \otimes \mathscr{I} \mathscr{I} W_{2}=\mathscr{I} W_{1}=\mathbb{Q}$.

$\mathrm{C}$ другой стороны, по лемме Нётер о нормализации, $\left(W_{1} \otimes \mathscr{I} W_{2}\right) \otimes \mathscr{I} \mathbb{Q}=$ $\mathscr{I}\left(W_{1} \otimes W_{2}\right)$ содержит подмодули, изоморфные $C_{k}(X)$ для любой кривой $X$ над $k$.

Лемма 4.2.1 [7; леммы 6.27, 6.28]. Пусть $n=\infty$. Тогда для любого конечного набора гладких неприводимых собственных $k$-многообразий $X_{1}, \ldots, X_{N}$ имеется канонический сюргективный морфизм $\mathscr{I}(\alpha): C_{k\left(X_{1} \times{ }_{k} \cdots \times{ }_{k} X_{N}\right)} \rightarrow$ $\mathscr{I}\left(C_{k\left(X_{1}\right)} \otimes \cdots \otimes C_{k\left(X_{N}\right)}\right)$ представлений $G$.

Если $C_{k\left(X_{1} \times{ }_{k} \cdots \times{ }_{k} X_{N}\right)}=C H_{0}\left(X_{1} \times_{k} \cdots \times_{k} X_{N}\right)_{\mathbb{Q}}$, то $\mathscr{I}(\alpha)-$ изоморфизм.

Если $\mathscr{I}(\alpha)$ - изоморфизм, то $\otimes_{\mathscr{I}}$ ассоциативна, класс проективных обгектов $\mathscr{I}_{G}$ замкнут относительно $\otimes_{\mathscr{I}} u W_{1} \otimes \mathscr{I} \cdots \otimes \mathscr{I} W_{N}=\mathscr{I}\left(W_{1} \otimes \cdots \otimes W_{N}\right)$.

“Формула Кюннета" для произведений с кривыми. Отображение ограничения $\left.\left.\tau \mapsto \tau\right|_{k(X)} \otimes \tau\right|_{k(Y)}$ определяет гомоморфизм $G$-модулей $\mathbb{Q}\left[\left\{k(X) \otimes_{k}\right.\right.$ $k(Y) \stackrel{/ k}{\hookrightarrow} F\}] \stackrel{\alpha}{\longrightarrow} C_{k(X)} \otimes C_{k(Y)}$. Из леммы 4.2 .1 следует соръективность $\alpha$, что задает сюръекцию $C_{k\left(X \times_{k} Y\right)} \rightarrow C_{k(X)} \otimes \mathscr{I} C_{k(Y)}$.

Для произвольных $A \in C_{k(X)}$ и $B \in C_{k(Y)}$ выберем $\widetilde{A} \in \mathbb{Q}[\{k(X) \stackrel{/ k}{\hookrightarrow} F\}]$ и $\widetilde{B} \in \mathbb{Q}[\{k(Y) \stackrel{/ k}{\hookrightarrow} F\}]$ такие, что все вложения из $\widetilde{A}$ и из $\widetilde{B}-$ в попарно общем положении. ${ }^{8}$

Нужно проверить, что класс $\widetilde{A} \times \widetilde{B} \in \mathbb{Q}\left[\left\{k\left(X \times_{k} Y\right) \stackrel{/ k}{\hookrightarrow} F\right\}\right]$ в $C_{k\left(X \times_{k} Y\right)}$ не зависит от выбора $\widetilde{A}$ и $\widetilde{B}$. Если $\widetilde{A}^{\prime} \in \mathbb{Q}[\{k(X) \stackrel{/ k}{\hookrightarrow} F\}]$ и $\widetilde{B}^{\prime} \in \mathbb{Q}[\{k(Y) \stackrel{/ k}{\hookrightarrow} F\}]$ определены аналогично, то выберем $\widetilde{B}^{\prime \prime} \in \mathbb{Q}[\{k(Y) \stackrel{/ k}{\hookrightarrow} F\}]$ такой, что все вложения из $\widetilde{A}$ и из $\widetilde{B}^{\prime \prime}$, а также из $\widetilde{A}^{\prime}$ и из $\widetilde{B}^{\prime \prime}-$ в попарно общем положении. Тогда $\widetilde{A} \times \widetilde{B}-\widetilde{A}^{\prime} \times \widetilde{B}^{\prime}=\left(\widetilde{A}-\widetilde{A}^{\prime}\right) \times \widetilde{B}^{\prime \prime}+\widetilde{A} \times\left(\widetilde{B}-\widetilde{B}^{\prime \prime}\right)+\widetilde{A}^{\prime} \times\left(\widetilde{B^{\prime \prime}}-\widetilde{B}^{\prime}\right)$.

Таким образом, нужно проверить условие $\star_{X, Y}$ :

\footnotetext{
${ }^{8}$ Сначала выберем $\widetilde{A}$ и $\widetilde{B}$ произвольно. Проведем через каждую точку $P$ носителя $\widetilde{B}$ общую кривую $C_{P}$, на которой точка $P$ - общая относительно некоторого поля определения $C_{P}$. Заменим $P$ линейно эквивалентной линейной комбинацией точек $C_{P}$ в общем положении относительно $\widetilde{A}$. Получим требуемое $\widetilde{B}$.
} 
если класс $\sum_{i=1}^{N} a_{i} \tau_{i} \in \mathbb{Q}[\{k(X) \stackrel{/ k}{\hookrightarrow} F\}]-$ нулевой в $C_{k(X)}$ и все $\tau_{i}$ - в общем положении относительно $\sigma: k(Y) \stackrel{/ k}{\hookrightarrow} F$, то класс $\gamma:=\sum_{i=1}^{N} a_{i}\left(\tau_{i}, \sigma\right) \in \mathbb{Q}\left[\left\{k\left(X \times_{k} Y\right) \stackrel{/ k}{\hookrightarrow} F\right\}\right]-$ нулевой в $C_{k\left(X \times_{k} Y\right)}$, а также условие $\star_{Y, X}$.

По определению функтора $\mathscr{I}$, найдутся чисто трансцендентные расширения $L_{j}^{\prime} \mid L_{j}$, элементы $\alpha_{j} \in \mathbb{Q}[\{k(X) \stackrel{/ k}{\hookrightarrow} F\}]^{G_{F \mid L_{j}^{\prime}}}$ и $\xi_{j} \in G_{F \mid L_{j}}$ такие, что $\sum_{i=1}^{N} a_{i} \tau_{i}=$ $\sum_{j}\left(\xi_{j} \alpha_{j}-\alpha_{j}\right)$

Если $\sigma$ - в общем положении относительно композита $L$ всех $\tau_{i}(k(X))$, то найдется $\kappa \in G_{F \mid L}$ такой, что $\kappa \sigma=: \sigma^{\prime}$ - в общем положении относительно композита всех $L_{j}^{\prime}$. Тогда $\gamma^{\prime}:=\kappa \gamma=\sum_{i} a_{i}\left(\tau_{i}, \sigma^{\prime}\right)=\sum_{j}\left(\xi_{j} \alpha_{j}-\alpha_{j}\right) \otimes \sigma^{\prime}$. Положим $K_{j}:=$ $L_{j} \sigma^{\prime}(k(Y))$ и $K_{j}^{\prime}:=L_{j}^{\prime} \sigma^{\prime}(k(Y))$. Тогда $\alpha_{j} \otimes \sigma^{\prime} \in \mathbb{Q}\left[\left\{k\left(X \times_{k} Y\right) \stackrel{/ k}{\hookrightarrow} F\right\}\right]^{G_{F \mid K_{j}^{\prime}}}$, $K_{j}^{\prime}$ - чисто трансцендентное расширение $K_{j}$ и найдутся такие $\xi_{j}^{\prime} \in G_{F \mid \sigma^{\prime}(k(Y))}$, что $\left.\xi_{j}^{\prime}\right|_{L_{j}^{\prime}}=\left.\xi_{j}\right|_{L_{j}^{\prime}}$. Отсюда следует, что $\gamma^{\prime}=\sum_{j}\left(\xi_{j}^{\prime}\left(\alpha_{j} \otimes \sigma^{\prime}\right)-\alpha_{j} \otimes \sigma^{\prime}\right)$ лежит, по определению функтора $\mathscr{I}$, в ядре проекции $\mathbb{Q}\left[\left\{k\left(X \times_{k} Y\right) \stackrel{/ k}{\hookrightarrow} F\right\}\right] \rightarrow C_{k\left(X \times_{k} Y\right)}$, а значит, то же верно и для $\gamma$.

Проверим, что условия $\star_{X, Y}$ и $\star_{Y, X}$ равносильны. Пусть общая кривая $C$ на $Y$ над некоторым полем, содержащим все $\tau_{i}(k(X))$, проходит через $\sigma$. Тогда $\sigma$ линейно эквивалентна линейной комбинации $\beta$ общих точек $C$ (которые являются общими точками $Y)$. Тогда образ $\gamma$ в $C_{k\left(X \times_{k} Y\right)}$ совпадает с образом $\sum_{i} a_{i} \tau_{i} \times(\sigma-\beta)$, что доказывает импликацию $\star_{Y, X} \Rightarrow \star_{X, Y}$.

ПримеР 4.2.2. Проверим условие $\star_{X, Y}$ в случае, когда $X$ - гладкая полная кривая. Пусть $K=\overline{\sigma(k(Y))}$. Тогда $\sum_{i} a_{i} \tau_{i}$ - общий дивизор на кривой $X_{K}$ над $K$, линейно эквивалентный нулю. Согласно [7; лемма 6.18$], G_{F \mid K}$-модуль общих дивизоров на $X_{K}$ над $K$, линейно эквивалентных нулю, порожден элементами $w_{M}=\sum_{j=1}^{M}\left(\sigma_{j}-\sigma_{j}^{\prime}\right)$ для всех $M \gg 0$, где $\left(\sigma_{1}, \ldots, \sigma_{M} ; \sigma_{1}^{\prime}, \ldots, \sigma_{M}^{\prime}\right)-$ общая $F$-точка слоя над нулем морфизма $X_{K}^{M} \times_{K} X_{K}^{M} \rightarrow \mathrm{Pic}^{\circ} X_{K}$, посылающего точку $\left(x_{1}, \ldots, x_{M} ; y_{1}, \ldots, y_{M}\right)$ в класс $\sum_{j=1}^{M}\left(x_{j}-y_{j}\right)$. Ясно, что композит всех подполей $\sigma_{j}(k(X)) \sigma_{j}^{\prime}(k(X))$ находится в общем положении по отношению к $K$. То же относится и к любому элементу в $G_{F \mid K}$-орбите $w_{M}$. Поэтому, как мы видели выше, образ $\sum_{i} a_{i}\left(\tau_{i}, \sigma\right)$ в $C_{k\left(X \times{ }_{k} Y\right)}-$ нулевой.

Таким образом, имеется каноническая сюръекция $C_{k(X)} \otimes C_{k(Y)} \rightarrow C_{k\left(X \times{ }_{k} Y\right)}$, по крайней мере если $X-$ кривая, и композиция $C_{k\left(X \times_{k} Y\right)} \rightarrow C_{k(X)} \otimes \mathscr{I}_{k} C_{k(Y)} \rightarrow$ $C_{k\left(X \times_{k} Y\right)}$ тождественна.

СЛЕДСТВИЕ 4.2.3. Если $n=\infty, X$ и $Y$ - неприводимъе $k$-многообразия u $X-к р и в а я$, mо $C_{k\left(X \times{ }_{k} Y\right)}=C_{k(X)} \otimes \mathscr{I} C_{k(Y)}$.

ПримеР 4.2.4. Пусть $X_{j}, 1 \leqslant j \leqslant N$, и $Y$ - неприводимые $k$-многообразия, причем все $X_{j}$, кроме, быть может, одного, - кривые. Пусть $Y \rightarrow X_{j}, 1 \leqslant$ $j \leqslant N,-$ доминантные отображения. Тогда имеется естественный морфизм $C_{k(Y)} \rightarrow C_{k\left(\prod_{j=1}^{N} X_{j}\right)}$. Действительно, в этом случае имеется естественный 
морфизм $\bigotimes_{j=1}^{N} C_{k\left(X_{j}\right)} \rightarrow C_{k\left(\prod_{j=1}^{N} X_{j}\right)}$, композиция которого с

$$
\mathbb{Q}[\{k(Y) \stackrel{/ k}{\hookrightarrow} F\}] \rightarrow \mathbb{Q}\left[\prod_{j=1}^{N}\left\{k\left(X_{j}\right) \stackrel{/ k}{\hookrightarrow} F\right\}\right]=\bigotimes_{j=1}^{N} \mathbb{Q}\left[\left\{k\left(X_{j}\right) \stackrel{/ k}{\hookrightarrow} F\right\}\right] \rightarrow \bigotimes_{j=1}^{N} C_{k\left(X_{j}\right)}
$$

пропускается через $C_{k(Y)}$. В предположении, что точен функтор $(-)_{v}$ из предложения 1.2.4 (см. также с. 146), можно построить естественный морфизм $C_{k(D)} \rightarrow C_{k(X)}$ для любого неприводимого дивизора $D$ на любом неприводимом многообразии $X$ над $k$, см. следствие 4.4.14.

4.3. Геометрическая конструкция допустимых представлений. Мы приступаем к построению некоторого запаса полупростых допустимых представлений группы $G$. Гипотетически, в случае $n=\infty$ все полупростые допустимые представления группы $G$ получаются этим способом.

Положим $B^{q}(X)=A^{q}(X)$, если $\sim$ - численная эквивалентность (над $\left.k !\right)$. Как и прежде, $X_{E}:=X \times_{k} E$ для любого $k$-многообразия $X$ и любого расширения полей $E \mid k$.

Напомним, что $B^{q}(X)$ - предел некоторых факторов $\mathbb{Q}$-векторных пространств классов численной эквивалентности циклов коразмерности $q$ на гладких собственных многообразиях над $k$, и ни в каком смысле не над $F$, даже если $X=Y_{F}$, см. текст после леммы 3.1.4.

Лемма 4.3.1. Пусть $W \in \mathscr{S} m_{G} . \quad$ Если $\operatorname{Hom}_{G}\left(Z^{\operatorname{dim} X}\left(k(X) \otimes_{k} F\right), W\right)=$ $\operatorname{Hom}_{G}\left(C H_{0}\left(X_{F}\right), W\right)$ для любого гладкого собственного многообразия $X$ над $k$, то $W$ полупрост тогда и только тогда, когда полупросты $W^{G_{F \mid F^{\prime}}}$ как

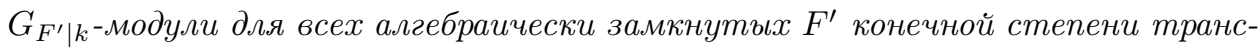
цендентности над $k$.

ДоказАТЕльство. Ясно, что $W \in \mathscr{I}_{G}$. По предложению $3.1 .1, G$-модуль $W$ полупрост тогда и только тогда, когда для любого $L \subset F$ конечного типа над $k$ и любого чисто трансцендентного расширения $L^{\prime} \mid L$ в $F$ с $\overline{L^{\prime}}=F$ модуль $W^{G_{F \mid L^{\prime}}}=W^{G_{F \mid L}}$ над алгеброй Гекке $\mathscr{H}_{G_{F \mid L^{\prime}}}=h_{L^{\prime}} \mathbb{D} h_{L^{\prime}} \supseteq\left\langle h_{L^{\prime}} \sigma h_{L^{\prime}} \mid \sigma \in G\right\rangle_{\mathbb{Q}}$ полупрост. Здесь $h_{L^{\prime}}$ - мера Хаара на $G_{F \mid L^{\prime}}$. Поскольку $W$ - фактор прямой суммы объектов вида $C H_{0}\left(X_{F}\right)_{\mathbb{Q}}$, действие $\mathbb{D} \otimes W^{G_{F \mid L}} \rightarrow \mathbb{Q}\left[G / G_{F \mid L}\right] \otimes$ $W^{G_{F \mid L}} \rightarrow W$ пропускается через $C H_{0}\left(Y_{F}\right)_{\mathbb{Q}} \otimes W^{G_{F \mid L}} \rightarrow W$, где $Y$ - гладкая собственная модель расширения $L \mid k$ (см. предложение 3.1.6). Поэтому действие $\mathscr{H}_{G_{F \mid L^{\prime}}}$ пропускается через $C H_{0}\left(Y_{k(Y)}\right)_{\mathbb{Q}}=h_{L^{\prime}} C H_{0}\left(Y_{F}\right)_{\mathbb{Q}}$.

Другими словами, действие алгебры $\mathscr{H}_{G_{F \mid L^{\prime}}}(G)$ на $W^{G_{F \mid L}}$ определяется действием алгебры $\mathscr{H}_{G_{\bar{L} \mid L}}\left(G_{\bar{L} \mid k}\right)$, так что полупростота $\mathscr{H}_{G_{F \mid L^{\prime}}}(G)$-модуля $W^{G_{F \mid L}}$ эквивалентна его полупростоте как $\mathscr{H}_{G_{\bar{L} \mid L}}\left(G_{\bar{L} \mid k}\right)$-модуля.

ПРЕДЛОЖЕНИЕ 4.3.2. G-модуль $\mathbf{B}_{X}^{q}:=B^{q}\left(X_{F}\right)$ допустим и полупрост для любого гладкого собственного $k$-многообразия $X$ и любого $q \geqslant 0$. Если $q \in\{0,1\}$ или $q=\operatorname{dim} X \leqslant n$, то $\mathbf{B}_{X}^{q}-$ конечной длины. 
ДокАЗАТЕЛЬСтво. По стандартным соображениям, можно считать, что $k$ вложено в поле комплексных чисел $\mathbb{C}$, и, значит, для любого гладкого неприводимого собственного $d$-мерного $k$-многообразия $Y$ с $k(Y)=: L \subset F$ пространство $B^{q}\left(X_{F}\right)^{G_{F \mid L}}$ - фактор конечномерного пространства $Z^{q}\left(X \times_{k} Y\right) / \sim_{\text {hom }} \subseteq$ $H^{2 q}\left(\left(X \times_{k} Y\right)(\mathbb{C}), \mathbb{Q}(q)\right)$, так что представление $B^{q}\left(X_{F}\right)$ допустимо.

Согласно предложению 3.1.6 и лемме 4.3.1 (где $W=B^{q}\left(X_{F}\right)$, и потому $\left.W^{G_{F \mid L^{\prime}}}=W^{G_{F \mid L}}=B^{q}\left(X_{L}\right)\right)$, полупростота $G$-модуля $B^{q}\left(X_{F}\right)$ эквивалентна полупростоте $\mathscr{H}_{G_{\bar{L} \mid L}}\left(G_{\bar{L} \mid k}\right)$-модуля $B^{q}\left(X_{L}\right)$ для любого $L \subset F$ как выше.

Ядро гомоморфизма $A^{q}\left(X \times_{k} Y\right) \rightarrow A^{q}\left(X_{k(Y)}\right)$ является $A^{d}\left(Y \times_{k} Y\right)$-подмодулем в $A^{q}\left(X \times_{k} Y\right)$, поскольку $\alpha \circ \beta=\operatorname{pr}_{13 *}\left(\operatorname{pr}_{12}^{*} \alpha \cdot \operatorname{pr}_{23}^{*} \beta\right)$ для его произвольного элемента $\alpha$ и для любого элемента $\beta \in A^{d}\left(Y \times_{k} Y\right)$, так что проекция на $Y$ носителя $\alpha \circ \beta$ содержится в $\operatorname{pr}_{2}\left(\left(D \times_{k} Y\right) \cap \operatorname{supp}(\beta)\right)$ для некоторого дивизора $D$ на $Y$, размерность которого равна $d-1$, и, значит, $D$ не может доминировать $Y$. Отсюда следует, что $A^{q}\left(X_{k(Y)}\right)$ имеет естественную структуру $A^{d}\left(Y \times_{k} Y\right)$-модуля.

Согласно [6], алгебра $B^{d}\left(Y \times_{k} Y\right)$ полупроста, так что $B^{d}\left(Y \times_{k} Y\right)$-модуль $B^{q}\left(X_{k(Y)}\right)$ также полупрост. По лемме о сдвиге 3.1 .5 , гомоморфизм колец $\mathscr{H}_{G_{\bar{L} \mid L}}\left(G_{\bar{L} \mid k}\right) \rightarrow B^{d}\left(Y \times_{k} Y\right)$, индуцированный отождествлением алгебры Гекке $\mathscr{H}_{G_{\bar{L} \mid L}}\left(G_{\bar{L} \mid k}\right)$ с алгеброй невырожденных соответствий на $Y$ (см. текст после леммы 3.1.3), сюръективен. Это задает структуру (полупростого) $\mathscr{H}_{G_{\bar{L} \mid L}}\left(G_{\bar{L} \mid k}\right)$ модуля на любом $B^{d}\left(Y \times_{k} Y\right)$-модуле.

Длина любого циклического полупростого $G$-модуля, в частности $\mathbf{B}_{X}^{\operatorname{dim}} X$ (лемма 3.1.5), конечна.

Из теорем Лефшеца о гиперплоском сечении и об $(1,1)$-классах следует, что $\mathbf{B}_{X}^{q}$ - подфактор $\mathbf{B}_{H}^{q}$ для любого гладкого $q$-мерного плоского сечения $H$ многообразия $X$, так что длина $\mathbf{B}_{X}^{q}$ также конечна.

СлеДСТВИе 4.3.3. $\operatorname{Hom}_{G}\left(B^{q}\left(L^{\prime} \otimes_{k} F\right), B^{p}\left(L \otimes_{k} F\right)\right)=0$ для любой пары полей $L, L^{\prime}$ конечного типа над $k c \operatorname{deg} \operatorname{tr}(L \mid k)=p, \operatorname{deg} \operatorname{tr}\left(L^{\prime} \mid k\right)=q u p \neq q$.

ДокАЗАТЕЛьСтво. Если $p>n$ или $q>n$, то по крайней мере один из модулей $B^{q}\left(L^{\prime} \otimes_{k} F\right)$ и $B^{p}\left(L \otimes_{k} F\right)$ - нулевой, так что можно считать, что $\max (p, q)$ не превосходит $n$. По предложению 4.3.2, $G$-модули $B^{q}\left(L^{\prime} \otimes_{k} F\right)$ и $B^{p}\left(L \otimes_{k} F\right)$ полупросты, так что $\operatorname{Hom}_{G}\left(B^{q}\left(L^{\prime} \otimes_{k} F\right), B^{p}\left(L \otimes_{k} F\right)\right)$ изоморфно $\operatorname{Hom}_{G}\left(B^{p}\left(L \otimes_{k} F\right)\right.$, $\left.B^{q}\left(L^{\prime} \otimes_{k} F\right)\right)$, и поэтому можно считать, что $p>q$. Тогда, по следствию 3.1.7, $\operatorname{Hom}_{G}\left(B^{q}\left(L^{\prime} \otimes_{k} F\right), B^{p}\left(L \otimes_{k} F\right)\right)=B^{p}\left(L \otimes_{k} L^{\prime}\right)=0$.

СлЕДСТвИЕ 4.3 .4 [7; следствие 3.12]. Для любого гладкого неприводимого многообразия $X$ размерности не въие $n+1$ над $k$ u $q \in\{0,1,2, \operatorname{dim} X\}$ существует единственный $G$-подмодуль в $\mathbf{B}_{X}^{q}=B^{q}\left(X \times_{k} F\right)$, изоморфный $B^{q}\left(k(X) \otimes_{k} F\right)$.

Для каждой открытой компактной подгруппы $U \subset G$ и гладкого неприводимого многообразия $Y$ над $k$ с $k(Y)=F^{U}$ определим полупростой $G$-модуль (конечной длины) $\mathbf{B}_{Z, Y}^{q}$ как минимальный среди таких, что $\mathscr{H}_{\mathbb{Q}}(U)$-модуль $\left(\mathbf{B}_{Z, Y}^{q}\right)^{U}$ изоморфен $B^{q}\left(Z \times_{k} Y\right)$. По предложению 3.1.1, он существует и единственен.

Лемма 4.3.5 [7; лемма 3.13]. Пусть $X, Y$ и $Z$ - гладкие неприводимые $k$-многообразия, $\operatorname{dim} X=\operatorname{dim} Y=n \geqslant \operatorname{dim} Z, u p, q \geqslant 0$ - целье. 
Тогда $\operatorname{Hom}_{\mathscr{H}_{\mathbb{Q}}(U)}\left(B^{q}\left(Z \times_{k} Y\right), B^{p}\left(k(X) \otimes_{k} k(Y)\right)\right)=0$, если или $q=\operatorname{dim} Z<p$, или $q=n u \operatorname{dim} Z<p$, или $q>n u p+q>\operatorname{dim} Z+n$, или $q<p u q \in\{0,1\}$.

ПредлОЖЕНИЕ 4.3.6 [7; предложение 3.14]. Пусть X и $Y$ - гладкие неприводимые многообразия над $k$ и или $q \in\{0,1,2\}$, или $q=\operatorname{dim} X=\operatorname{dim} Y$. Тогда имеется единственный подмодуль в $B^{q}\left(X \times_{k} Y\right)$ над $\left(B^{\operatorname{dim} X}\left(X \times_{k} X\right) \otimes\right.$ $\left.B^{\operatorname{dim} Y}\left(Y \times_{k} Y\right)^{\mathrm{op}}\right)$, изоморфный его фактору $B^{q}\left(k(X) \otimes_{k} k(Y)\right)$.

Единственность такого подмодуля вытекает из леммы 4.3.5. Существование следует из полупростоты модуля $B^{q}\left(X \times_{k} Y\right)$, см. [6].

Представления, контрагредиентные к мотивным. Для каждой открытой компактной подгруппы $U$ в $\mathfrak{G}$ (см. п. 2.5) зафиксируем гладкое собственное неприводимое многообразие $Y_{U}$ над $k$ и вложение его поля функций в $F$ такие, что $F^{U}=k\left(Y_{U}\right) L_{m}$ для некоторого целого $m \geqslant 1$, где $k\left(Y_{U}\right)$ и $L_{m}$ алгебраически независимы над $k$, т. е. $\operatorname{dim}_{k} Y_{U}=m-1$. Пусть $n_{U}:=\operatorname{dim}_{k} Y_{U}$. Если $Y_{U}^{\prime}-$ другое многообразие с теми же свойствами, то существует канонический изоморфизм $B^{n_{U}^{\prime}}\left(\left(Y_{U}^{\prime}\right)_{L}\right)=B^{n_{U}}\left(\left(Y_{U}\right)_{L}\right)$, индуцированный изоморфизмами прямого образа вида $B^{n_{U}}\left(\left(Y_{U}\right)_{L}\right)=B^{n_{U}+q}\left(\left(Y_{U} \times \mathbb{P}^{q}\right)_{L}\right)$.

Для каждой упорядоченной пары открытых компактных подгрупп $U \supseteq U^{\prime}$ в $\mathfrak{G}$ можно выбрать гладкие собственные неприводимые многообразия $Y_{U}, Y_{U^{\prime}}$ над $k$ и вложения их полей функций в $F$ такие, что: (а) $F^{U}=k\left(Y_{U}\right) L_{m}$ и $F^{U^{\prime}}=k\left(Y_{U^{\prime}}\right) L_{m}$ для некоторого целого $m \geqslant 1$ и (б) $k\left(Y_{U}\right) k\left(Y_{U^{\prime}}\right)=k\left(Y_{U^{\prime}}\right)$ и $L_{m}$ алгебраически независимы над $k$. Тогда гомоморфизм обратного образа индуцирует каноническое вложение $B^{n_{U}}\left(\left(Y_{U}\right)_{L}\right) \hookrightarrow B^{n_{U^{\prime}}}\left(\left(Y_{U^{\prime}}\right)_{L}\right)$.

Это позволяет образовать индуктивную систему $\left(B^{n_{U}}\left(\left(Y_{U}\right)_{k(X)}\right)\right)_{U}$, где $U$ пробегает множество открытых компактных подгрупп в $\mathfrak{G}$.

СЛЕДСТВИЕ 4.3.7. Пусть $X$ и $Y$-гладкие неприводимые собственнъе $k$-многообразия. Тогда $\mathbb{Q}$-векторные пространства $B^{\operatorname{dim} X}\left(X_{k(Y)}\right)$ u $B^{\operatorname{dim} Y}\left(Y_{k(X)}\right)$ естественно двойственны друг другу. Если $\operatorname{dim} X \leqslant n$, то эта двойственность индуцирует невырожденное $\mathfrak{G}$-эквивариантное спаривание

$$
B^{\operatorname{dim} X}\left(X_{F}\right) \otimes \lim _{U} B^{n_{U}}\left(\left(Y_{U}\right)_{k(X)}\right) \rightarrow B^{\operatorname{dim} X}\left(X_{k(X)}\right)(\chi) \stackrel{\operatorname{tr}}{\longrightarrow} \mathbb{Q}(\chi) .
$$

ДоказАтельство. Пусть $n \geqslant \operatorname{dim} Y \geqslant \operatorname{dim} X$. По предложению 3.1.5, $B^{\operatorname{dim} X}\left(X_{k(Y)}\right)=\operatorname{Hom}_{G}\left(B^{\operatorname{dim} Y}\left(Y_{F}\right), B^{\operatorname{dim} X}\left(X_{F}\right)\right)$ и аналогично, $B^{\operatorname{dim} Y}\left(Y_{k(X)}\right)=$ $\operatorname{Hom}_{G}\left(B^{\operatorname{dim} X}\left(X_{F}\right), B^{\operatorname{dim} Y}\left(Y_{F}\right)\right)$. Согласно предложению 4.3.2, представления $B^{\operatorname{dim} Y}\left(Y_{F}\right)$ и $B^{\operatorname{dim} X}\left(X_{F}\right)$ группы $G$ полупросты, и их длины конечны. Для любых $\alpha \in B^{\operatorname{dim} X}\left(X_{k(Y)}\right)$ и $\beta \in B^{\operatorname{dim} Y}\left(Y_{k(X)}\right)$ определим элемент $\alpha \circ \beta \in$ $\operatorname{End}_{G}\left(B^{\operatorname{dim} X}\left(X_{F}\right)\right)=B^{\operatorname{dim} X}\left(X_{k(X)}\right)$ как композицию соответствующих $G$-гомоморфизмов $\alpha$ и $\beta$. Если $\pi: Z \rightarrow Y-$ морфизм, конечный в общей точке, то обозначим через $\alpha^{\prime}$ и $\beta^{\prime}$ образы $\alpha$ и $\beta$ в $B^{\operatorname{dim} X}\left(X_{k(Z)}\right)$ и $B^{\operatorname{dim} Y}\left(Z_{k(X)}\right)$ соответственно, т. е. $\alpha^{\prime}=\alpha \circ \pi_{*}$ и $\beta^{\prime}=\pi^{*} \circ \beta$. По формуле проекции $\alpha^{\prime} \circ \beta^{\prime}=$ $\operatorname{deg} \pi \cdot \alpha \circ \beta$. Положим $\langle\alpha \cdot \beta\rangle=\operatorname{tr}(\alpha \circ \beta)(=\operatorname{tr}(\beta \circ \alpha))$. Если $\alpha \neq 0$, то существует элемент $\gamma \in B^{\operatorname{dim} Y}\left(Y_{k(X)}\right)$ такой, что $\alpha \circ \gamma-$ ненулевой проектор в $\operatorname{End}_{G} B^{\operatorname{dim} X}\left(X_{F}\right)$, так что форма $\alpha \otimes \beta \mapsto\langle\alpha \cdot \beta\rangle$ невырождена. Определим форму $B^{\operatorname{dim} X}\left(X_{F}\right) \otimes \lim _{U} B^{n_{U}}\left(\left(Y_{U}\right)_{k(X)}\right) \rightarrow \mathbb{Q}(\chi)$ как $\alpha \otimes \beta \mapsto\langle\alpha \cdot \beta\rangle \cdot[U]$ для 
любых $\alpha \in B^{\operatorname{dim} X}\left(X_{F}\right)^{U}$ и $\beta \in B^{n_{U}}\left(\left(Y_{U}\right)_{k(X)}\right)$. По формуле проекции, это определение корректно.

Подчеркнем, что $B^{*}\left(X_{F}\right)$ - допустимое представление группы $\mathfrak{G}$ (см. предложение 4.6.1). В частности, чистые эффективные мотивы образуют полную подкатегорию в категории градуированных полупростых допустимых $\mathfrak{G}$-модулей. Заметим, что, поскольку категория градуированных полупростых допустимых $\mathfrak{G}$-модулей конечной длины самодвойственна, произвольные чистые мотивы (не обязательно эффективные) можно реализовать в этой категории.

Проектор $\Delta_{k(X)}$. Для любой пары многообразий $X, Y$ пусть $\alpha \mapsto{ }^{t} \alpha-$ транспозиция циклов, индуцированная транспозицией $X \times Y \stackrel{\sim}{\longrightarrow} Y \times X$. Обозначим через $\Delta_{k(X)}={ }^{t} \Delta_{k(X)}$ тождественный (диагональный) элемент алгебры $B^{\operatorname{dim} X}\left(k(X) \otimes_{k} k(X)\right)$, рассматриваемый как элемент кольца $B^{\operatorname{dim} X}\left(X \times_{k} X\right)$.

Лемма 4.3 .8 [7; леммы 3.15, 3.16]. Для любого неприводимого гладкого собственного многообразия $X$ над $k$ элемент $\Delta_{k(X)}$ - централъный проектор алгебры $B^{\operatorname{dim} X}\left(X \times_{k} X\right)$. Левъй (эквивалентно, правый) идеал, порожденнъй $\Delta_{k(X)}$, совпадает с (образом колъца) $B^{\operatorname{dim} X}\left(k(X) \otimes_{k} k(X)\right)$. Модуль $\Delta_{k(X)} B^{\operatorname{dim} X}\left(X_{L}\right)$ совпадает с $B^{\operatorname{dim} X}\left(k(X) \otimes_{k} L\right)$ для любого расширения полей $L \mid k$.

ПРЕДЛОжЕНИЕ 4.3 .9 [7; предложение 3.17]. $\left(X, \Delta_{k(X)}\right)$ - максималъныц примитивный $n$-подмотив мотива $\left(X, \Delta_{X}\right)$ для любого неприводимого гладкого собственного $n$-мерного $k$-многообразия $X$. Мотив $\left(X, \Delta_{k(X)}\right)$ - бирациональный инвариант $X$.

Бирациональная инвариантность $\left(X, \Delta_{k(X)}\right)$ следует из бирациональной инвариантности $Y^{\text {prim }}$ для любого гладкого проективного многообразия $Y$ над $k$, см. ниже.

СлеДСтвиЕ 4.3.10 [7; следствие 3.18]. Пусть $X$ и $Y$ - гладкие неприводимые собственные многообразия над $k u \operatorname{dim} X=\operatorname{dim} Y=n$. Тогда

$$
\Delta_{k(X)} \cdot B^{n}\left(X \times_{k} Y\right)=B^{n}\left(X \times_{k} Y\right) \cdot \Delta_{k(Y)}=\Delta_{k(X)} \cdot B^{n}\left(X \times_{k} Y\right) \cdot \Delta_{k(Y)}
$$

- единственный $\left(B^{n}\left(X \times_{k} X\right) \otimes B^{n}\left(Y \times_{k} Y\right)^{\text {ор }}\right)$-подмодуль в $B^{n}\left(X \times_{k} Y\right)$, изоморфныи своему фактору $B^{n}\left(k(X) \otimes_{k} k(Y)\right)$. Аналогично, $B^{q}\left(k(X) \otimes_{k} k(Y)\right)=$ $\Delta_{k(X)} \cdot B^{q}\left(X \times_{k} Y\right) \cdot \Delta_{k(Y)}$ для $q=0,1$.

Функторы $\mathbb{B}^{\bullet}$ и $\mathfrak{B}^{q}$. Для гладкого проективного многообразия $Y$ над $k$ пусть мотив $Y^{\text {prim }}$ определен как пересечение ядер всех морфизмов $\varphi: Y \rightarrow$ $M \otimes \mathbb{L}$ для всевозможных эффективных мотивов $M$, или, что эквивалентно, $Y^{\text {prim }}$ - это коядро морфизма $\sum \varphi: \bigoplus_{M \otimes \mathbb{L} \stackrel{\varphi}{\longrightarrow} Y} M \otimes \mathbb{L} \rightarrow Y$ (с такими же $\left.M\right)$. Ясно, что $Y \mapsto Y^{\text {prim }}$ - функтор из категории гладких проективных многообразий в категорию чистых мотивов. Любое бирациональное отображение есть композиция раздутия и стягивания с гладкими центрами (см. [41], [42]). Поскольку раздутие не меняет $Y^{\text {prim }}$ (см. [43]), из этого следует, что $Y^{\text {prim }}$ инвариант поля функций $k(Y)$. Согласно теореме Хиронаки, для любого расширения конечного типа $L \mid k$ в $F$ существует гладкое проективное многообразие $Y_{[L]}$ над $k$ с полем функций $L$, и, следовательно, мы получаем каноническую 
проективную систему мотивов $\left\{Y_{[L]}^{\text {prim }}\right\}_{L}$, пронумерованную подполями $L$ в $F$ конечного типа над $k$.

Определим теперь функтор $\mathbb{B}^{\bullet}=\bigoplus \mathbb{B}^{[i]}$ в теореме 1.1 .5 из категории чистых мотивов в категорию градуированных $\mathbb{Q}$-векторных пространств, положив $\mathbb{B}^{[i]}=\lim _{L} \operatorname{Hom}\left(Y_{[L]}^{\text {prim }} \otimes \mathbb{L}^{\otimes i},-\right)$ для его компоненты степени $i$. Пусть также $\mathfrak{B}^{q}$ обозначает ограничение функтора $\mathbb{B}^{[0]}$ на подкатегорию примитивных $q$-мотивов. Группа $G$ действует на проективной системе $\left\{Y_{[L]}^{\mathrm{prim}}\right\}_{L}$ по правилу $Y_{[L]} \stackrel{\sigma}{\rightarrow} Y_{[\sigma(L)]}, \sigma(L) \stackrel{\sigma^{-1}}{\longrightarrow} L$, так что $G$ действует на пределах $\mathfrak{B}^{q}(M)$ и $\mathbb{B}^{\bullet}(M)$.

ЗАМЕЧАНИЕ 4.3.11. Любой чистый мотив $M=(X, \pi)$ изоморфен прямой сумме $\bigoplus_{0 \leqslant i, j, i+j \leqslant \operatorname{dim} X} M_{i j} \otimes \mathbb{L}^{\otimes i}$, где $M_{i j}$ - примитивный $j$-мотив и $\mathbb{L}=\left(\mathbb{P}^{1}\right.$, $\left.\mathbb{P}^{1} \times\{0\}\right)$, так что $\mathbb{B}^{[i]}(M) \cong \bigoplus_{j} \mathfrak{B}^{j}\left(M_{i j}\right)$. Это доказывается индукцией по размерности $d$ многообразия $X$ следующим образом. Пусть $M_{0 d}=\bigcap_{\varphi} \operatorname{ker}(\varphi)$, где $\varphi$ пробегает множество морфизмов из $M$ в мотивы вида $\left(Y \times \mathbb{P}^{1}, \Delta\right)$ для всех $Y \mathrm{c} \operatorname{dim} Y<d$. (По предложению 4.3.9, $M_{0 d}=\left(X, \pi \circ \Delta_{k(X)}\right)$.) Поскольку длина $M$ не превосходит $\operatorname{dim}_{\mathbb{Q}} \operatorname{End}(M)<\infty$, мотив $M / M_{0 d}$ можно вложить в конечную прямую сумму мотивов $\left(Y_{j} \times \mathbb{P}^{1}, \Delta\right)$ с $\operatorname{dim} Y_{j}<d$. Поскольку $\left(Y_{j} \times \mathbb{P}^{1}, \Delta\right)=\left(Y_{j}, \Delta\right) \oplus\left(Y_{j}, \Delta\right) \otimes \mathbb{L}$, индукция завершена. В действительности, разложение $M=\bigoplus_{0 \leqslant i, j, i+j \leqslant \operatorname{dim} X} \widetilde{M}_{i j}$, где $\widetilde{M}_{i j}$ изоморфен $M_{i j} \otimes \mathbb{L}^{\otimes i},-$ каноническое, так как $\widetilde{M}_{i j}$ - это сумма образов всех морфизмов $\varphi: N \otimes \mathbb{L}^{\otimes i} \rightarrow M$ для всевозможных примитивных $j$-мотивов $N$.

ПреДлОЖЕНИЕ 4.3.12 [7; предложение 3.19]. Если $\operatorname{dim} X=q \leqslant n u M=$ $(X, \pi)$ - примитивный q-мотив, то $\mathfrak{B}^{q}(M)=\mathbb{B}^{[0]}(M)=\pi B^{q}\left(X_{F}\right)$.

Доказательство использует эквивариантную версию разрешения особенностей и основную теорему работы [6].

Из предложения 4.3.12 и леммы 4.3.8 вытекает следующее утверждение.

СлеДСтвиЕ 4.3.13 [7; следствие 3.20]. Для любого $q<d:=\operatorname{dim} X$, любого неприводимого гладкого собственного $k$-многообразия $X$ и любого неприводимого гладкого $k$-многообразия $Y$ имеется равенство $\Delta_{k(X)} B^{d}\left(X \times_{k} Y\right)=$ $B^{d}\left(k(X) \otimes_{k} k(Y)\right)$, и группа $\Delta_{k(X)} B^{q}\left(X \times_{k} Y\right)$ обращается в нуль.

\section{"Поляризация" на $B^{n}\left(k(X) \otimes_{k} F\right)$ и поляризуемые $G$-модули.}

ПредлОжениЕ 4.3.14 [7; предложение 3.21]. Для любого неприводимого $k$-многообразия $X$ размерности $n$ существует симметрическое $G$-эквивариантное невырожденное спаривание

$$
B^{n}\left(k(X) \otimes_{k} F\right) \otimes B^{n}\left(k(X) \otimes_{k} F\right) \stackrel{\langle\cdot, \cdot\rangle}{\longrightarrow} \mathbb{Q}(\chi)
$$

такое, что $\left\langle p^{*}(\cdot), \cdot\right\rangle=\left\langle\cdot, p_{*}(\cdot)\right\rangle$ для любого рачионального отображения $p, \kappa о-$ нечного в общей точке. В частности, $\langle\cdot, \cdot\rangle$ индуцирует невырожденное спаривание между подмодулями $W:=\pi B^{n}\left(k(X) \otimes_{k} F\right) u^{t} W:={ }^{t} \pi B^{n}\left(k(X) \otimes_{k} F\right)$ для всех проекторов $\pi \in B^{n}\left(k(X) \otimes_{k} k(X)\right)$.

Если для $(n-1)$-циклов на $2 n$-мерных комплексных многообразиях численная эквивалентность совпадает с гомологической, то форма $\langle\cdot, \cdot\rangle$ является $(-1)^{n}$-определенной. Например, это заведомо верно при $n \leqslant 2$. 
Форма $\langle\alpha, \gamma\rangle \in \mathbb{Q}(\chi)$ определяется как $\langle\hat{\alpha} \cdot \hat{\gamma}\rangle \cdot[U]$, где $\alpha, \gamma \in B^{n}\left(k(X) \otimes_{k} F\right)$ фиксированы компактной открытой подгруппой $U \subset G, \hat{\alpha}, \hat{\gamma}$ - образы $\alpha, \gamma \in$ $B^{n}\left(k(X) \otimes_{k} k\left(Y_{U}\right)\right)$ в $B^{n}\left(X \times_{k} Y_{U}\right)$ в смысле предложения 4.3.6. Здесь $Y_{U}-$ гладкое собственное многообразие над $k$ с полем функций $k\left(Y_{U}\right)$, отождествленным с $F^{U}$, и $\alpha \otimes \beta \mapsto\langle\alpha \cdot \beta\rangle$ - форма пересечения на $B^{n}\left(X \times_{k} Y_{U}\right)$. По формуле проекции, $\langle\alpha, \gamma\rangle$ не зависит от произвола во всевозможных выборах, и $\left\langle p^{*}(\cdot), \cdot\right\rangle=\left\langle\cdot, p_{*}(\cdot)\right\rangle$.

Оставшаяся часть доказательства использует стандартную теорию пересечений, лемму 4.3 .5 и теорему Ходжа об индексе.

4.4. Нормирования и связанные с ними функторы. (Большая часть результатов этого пункта содержится в [19].) В этом пункте каждому гладкому представлению $G$ сопоставляется пучок в гладкой топологии на $k$. Для этого каждому гладкому $k$-многообразию $X$, его схемной точке $p \in X$ и вложению $k\left(X_{p}\right) \stackrel{/ k}{\hookrightarrow} F$ сопоставляется набор $J_{X, p}$ подполей в $F$ и определяются слои $\mathscr{W}_{X, p}:=W^{G_{F \mid k\left(X_{p}\right)}} \cap\left(\sum_{F^{\prime \prime} \in J_{X, p}} W^{G_{F \mid F^{\prime \prime}}}\right)$.

Естественно, что для каждого $q \geqslant 0$ из гладкого представления $\Omega_{F \mid k}^{q}$ группы $G$ мы хотим получить пучок $\Omega_{\mathscr{O} \mid k}^{q}$. Для представления $\bigotimes_{F}^{q} \Omega_{F \mid k}^{1}$ имеется два варианта: один - "гомотопически инвариантный" (и более естественный) $\bigotimes_{\mathscr{O}}^{q} \Omega_{\mathscr{O} \mid k}^{1}$, а другой - со свойством спуска Галуа. "Гомотопически инвариантный" означает, что для любого проективного расслоения $X \rightarrow Y$ над собственной базой $Y$ индуцированное отображение сечений - изоморфизм.

Функтор “глобализации”. Для любого набора $J$ подполей $F^{\prime \prime} \subset F$ аддитивный функтор $\Phi_{J}: W \mapsto \sum_{F^{\prime \prime} \in J} W^{G_{F \mid F^{\prime \prime}}}$ на категории $\mathscr{S} m_{G}$ сохраняет сюръекции, если любой элемент $J$ содержится в элементе $J$ сколь угодно большой конечной или счетной степени трансцендентности над $k$, и инъекции в общем случае.

ЗАмЕчАниЕ 4.4.1. Если набор $J$ состоит из всех чисто трансцендентных расширений поля $k$, то $\Phi_{J}\left(\Omega_{F \mid k}^{q}\right)=\Omega_{F \mid k}^{q}$ при $n>q$ и $\Phi_{J}\left(\Omega_{F \mid k, \text { рег }}^{q}\right)=0$ для любого $q \geqslant 1$. Поэтому $\Phi_{J}$, вообще говоря, не точен, даже если $n=\infty$.

В частности, с каждым кольцом дискретного нормирования $\mathscr{O}_{v} \in \mathscr{P}_{F}^{r}$ связан набор $J$ всех его подполей. Мы рассмотрим соответствующий функтор $\Phi_{J}$ :

$$
\begin{gathered}
(-)_{v}: \mathscr{S} m_{G} \rightarrow \mathscr{S} m_{G_{v}}, \\
W \mapsto W_{v}:=\sum_{\sigma \in G_{v}} W^{G_{F \mid \sigma\left(F^{\prime}\right)}}=\sum_{\sigma \in G_{v}^{\dagger}} W^{G_{F \mid \sigma\left(F^{\prime}\right)}} \subseteq W,
\end{gathered}
$$

где используются обозначения п. 2.4. Положим $\Gamma_{r}(W):=\bigcap_{\mathscr{O}_{v} \in \mathscr{P}_{F}^{r}} W_{v}$ и $\Gamma:=\Gamma_{1}$, т. е. $\Gamma_{r}$ - аддитивные функторы из категории $\mathscr{S} m_{G}$ в себя.

ПримеР 4.4.2. Имеют место равенства $\mathbb{Q}[\{L \stackrel{/ k}{\hookrightarrow} F\}]_{v}=\mathbb{Q}\left[\left\{L \stackrel{/ k}{\hookrightarrow} \mathscr{O}_{v}\right\}\right]$ и $(F[\{L \stackrel{/ k}{\hookrightarrow} F\}])_{v}=\mathscr{O}_{v}\left[\left\{L \stackrel{/ k}{\hookrightarrow} \mathscr{O}_{v}\right\}\right]$ (и все эти модули - нулевые при $\operatorname{degtr}(L \mid k)>$ $n-r) ; \Gamma(\mathbb{Q}[\{L \stackrel{/ k}{\hookrightarrow} F\}])=\Gamma(F[\{L \stackrel{/ k}{\hookrightarrow} F\}])=0$, если $L \neq k$. 
ЛЕмма 4.4.3. Если $n=\infty$, то имеются канонические изоморфизмы

$$
\operatorname{Hom}_{G}\left(W, W^{\prime}\right) \stackrel{\sim}{\longrightarrow} \operatorname{Hom}_{G_{v}}\left(W_{v}, W^{\prime}\right)=\operatorname{Hom}_{G_{v}}\left(W_{v}, W_{v}^{\prime}\right)
$$

для любых $W, W^{\prime} \in \mathscr{S} m_{G}$. В частности, функтор $(-)_{v}: \mathscr{S} m_{G} \rightarrow \mathscr{S} m_{G_{v}}$ вполне строг.

ЗАмечАниЕ 4.4.4. Ясно, что функтор $(-)_{v}$ не сохраняет неприводимость: как правило, сюръекция $W_{v} \rightarrow H_{0}\left(G_{v}^{\dagger}, W_{v}\right)$ нетривиальна и неинъективна. Например, длина $G_{v}$-модуля $F / k$ равна $r+1:(F / k)_{v}=\mathscr{O}_{v} / k \supsetneqq \mathfrak{m}_{v}=\mathfrak{p}_{1} \supsetneqq \mathfrak{p}_{2} \supsetneqq$ $\cdots \supsetneqq \mathfrak{p}_{r}$.

Лемма 4.4.5. Пусть $J-$ набор алгебраически замкнутых подполей $F^{\prime \prime} \subset F$ счетной степени трансцендентности над $k$. Точность $\Phi_{J}$ эквивалентна любому из следующих условий на $J$ :

- для любого иелого $N \geqslant 1$, любого расширения $L$ поля $k$ конечного типа, любого набора вложений $\xi_{j}: L \stackrel{/ k}{\hookrightarrow} F_{j}$ таких, что $F_{j} \in J$ для всех $1 \leqslant j \leqslant N$, и любого $\sigma: L \stackrel{/ k}{\hookrightarrow} F$ существует элемент $\alpha \in \mathbb{Q}[G]$ такой, что $\alpha \xi_{j}=0$ для всех $1 \leqslant j \leqslant N u \alpha \sigma-\sigma \in \mathbb{Q}\left[\left\{L \stackrel{/ k}{\hookrightarrow} F^{\prime \prime} \mid F^{\prime \prime} \in J\right\}\right]$;

- для любого неприводимого $k$-многообразия $X$, любого целого $N \geqslant 1$, любого набора доминантных и не конечных в общей точке $k$-морфизмов $f_{j}: X \rightarrow Y_{j}$, $0 \leqslant j \leqslant N$, таких, что $f_{0}$ не пропускается через $f_{j}$ ни длякакого $1 \leqslant j \leqslant N, u$ любой общей точки $\sigma: k\left(Y_{0}\right) \stackrel{/ k}{\hookrightarrow} F$ существует общий 0 -цикл $\alpha \in X(F)$ такой, ито $\left(f_{j}\right)_{*} \alpha=0$ для всех $1 \leqslant j \leqslant N u\left(f_{0}\right)_{*} \alpha-\sigma \in \mathbb{Q}\left[\left\{k\left(Y_{0}\right) \stackrel{/ k}{\hookrightarrow} F^{\prime \prime} \mid F^{\prime \prime} \in J\right\}\right]$.

Условия леммы 4.4.5 выполняются для $\sigma$ в общем положении относительно композита всех $\xi_{j}(L)$. Это и следующий факт дают основание ожидать, что функтор $(-)_{v}$ точен.

ПредЛОЖЕНИЕ 4.4.6. Пусть $H$ - алгебраическая $k$-группа, $N \geqslant 1$ - целое u $H_{i}-k$-подгруппа для каждого $0 \leqslant i \leqslant N$. Предположим, что $H_{j}$ нормализует $H_{i}$ для каждой пары $0 \leqslant i<j \leqslant N$ u $H_{i}$ не содержится в $H_{0}$ ни для одного $1 \leqslant i \leqslant N$. Обозначим через $f_{i}: H \rightarrow H / H_{i}$ соответствующие проекиии. Тогда существует 0 -иикл $\alpha \in \mathbb{Q}[H(F)]$ такой, что $\left(f_{i}\right)_{*} \alpha=0$ для любого $1 \leqslant i \leqslant N u\left(f_{0}\right)_{*} \alpha \neq 0$. Более явно, почти все 0 -ицкль вида $\left(h_{1}-1\right) \cdots\left(h_{N}-1\right)$, где $h_{i} \in H_{i}$ для всех $1 \leqslant i \leqslant N$, удовлетворяют этим условиям.

Обозначим через $\mathscr{I}=\mathscr{I}_{/ k}=\mathscr{I}_{F \mid k}: \mathscr{S}_{G} \rightarrow \mathscr{I}_{G}$ левый сопряженный к функтору включения $\mathscr{I}_{G} \hookrightarrow \mathscr{S} m_{G}$, и положим $C_{L}:=\mathscr{I}_{F \mid k} \mathbb{Q}[\{L \stackrel{/ k}{\hookrightarrow} F\}]$ для любого конечно порожденного расширения $L \mid k$, см. теорему $1.1 .10,4)$.

ЛЕмма 4.4.7. При $n=\infty u r=1$ проекиия $\mathbb{Q}\left[\left\{k(X) \stackrel{/ k}{\hookrightarrow} \mathscr{O}_{v}\right\}\right] \rightarrow C_{k(X)}$ сюроективна для любого гладкого полного неприводимого многообразия $X$ над $k$. $B$ частности, $\left(C_{k(X)}\right)_{v}=C_{k(X)} u W_{v}=W\left(\right.$ а значит, $\left.\Gamma(W)=W_{v}=W\right)$ для всех $W \in \mathscr{I}_{G}$. 
ЛЕмма 4.4.8. Предположим, ито $n=\infty$. Тогда $\left(W_{1} \otimes W_{2}\right)_{v} \subseteq\left(W_{1}\right)_{v} \otimes\left(W_{2}\right)_{v}$ $u \Gamma\left(W_{1} \otimes W_{2}\right) \subseteq \Gamma\left(W_{1}\right) \otimes \Gamma\left(W_{2}\right)$ для любих $W_{1}, W_{2} \in \mathscr{S}_{m_{G}}$. Однако $(W \otimes W)_{v} \neq$ $W_{v} \otimes W_{v}$, если $W=\mathbb{Q}[F \backslash k]$. Если выполнено одно из условий: или $W_{1}-$ бактор $A(F)$ для некоторой коммутативной алгебраической $k$-группы $A$, или $W_{1} \in \mathscr{I}_{G}$, mо $\left(W_{1} \otimes W_{2}\right)_{v}=\left(W_{1}\right)_{v} \otimes\left(W_{2}\right)_{v}$ для любого $W_{2} \in \mathscr{S} m_{G}$.

ЗАмечАнИЕ 4.4 .9 . (i) Если $W$ имеет структуру $F$-векторного пространства $F \otimes W \rightarrow W$, то, по лемме 4.4.8, $W_{v}$ обладает структурой $\mathscr{O}_{v}$-модуля: $(F \otimes W)_{v}=$ $\mathscr{O}_{v} \otimes W_{v} \rightarrow W_{v}$. Ясно, что $F \otimes_{\mathscr{O}_{v}} W_{v} \rightarrow W$ инъективно, но не сюръективно, как показывает пример $W=F[\{L \stackrel{/ k}{\hookrightarrow} F\}]$.

(ii) Ясно, что $\Gamma_{r}$ сохраняет инъекции, но не сюръекции. А именно, пусть $W:=\bigotimes_{k}^{N} F \rightarrow \Omega_{F \mid k}^{N-1}$ задано формулой $a_{1} \otimes \cdots \otimes a_{N} \mapsto a_{1} d a_{2} \wedge \cdots \wedge d a_{N}$. Тогда $W_{v}=\bigotimes_{k}^{N} \mathscr{O}_{v}$, если $n \geqslant 2 N$, так что $\left(\bigotimes_{F}^{N-1} \Omega_{F \mid k_{0}}^{1}\right)_{v}=\bigotimes_{F}^{N-1} \Omega_{\mathscr{O}_{v} \mid k_{0}}^{1}$ для любого $k_{0} \subseteq k ;$ и $\Gamma\left(\bigotimes_{k}^{N} F\right)=k$, но $\Gamma_{r}\left(\Omega_{F \mid k}^{\bullet}\right)=\Omega_{F \mid k, \text { рег }}^{\bullet}$ для любого $r \geqslant 1$ (см. [19]), а в случае $n=\infty$ можно также воспользоваться леммой 4.4.8.

Для целого нормального $k$-многообразия $X$ с $k(X) \subset F$ пусть $\mathfrak{V}(X)$ - множество всех таких дискретных нормирований поля $F$ ранга один, тривиальных на $k$, что их ограничения на $k(X)$ или тривиальны, или соответствуют дивизорам на $X$.

Положим $\mathscr{W}(X):=W^{G_{F \mid k(X)}} \cap \bigcap_{v \in \mathfrak{V}(X)} W_{v} \subseteq \mathscr{W}$.

Ясно, что если доминантный морфизм $U \rightarrow X$ переводит дивизоры на $U$ в дивизоры на $X$, то $\mathfrak{V}(U) \subseteq \mathfrak{V}(X)$, так что $\mathscr{W}(X) \subseteq \mathscr{W}(U)$.

Если $X=U_{1} \cup U_{2}$, то $\mathfrak{V}(X)=\mathfrak{V}\left(U_{1}\right) \cup \mathfrak{V}\left(U_{2}\right)$, поскольку $X^{1}=U_{1}^{1} \cup U_{2}^{1}$, так что $\mathscr{W}(X)=\mathscr{W}\left(U_{1}\right) \cap \mathscr{W}\left(U_{2}\right)$, т. е. $U \mapsto \mathscr{W}(U)$ для открытых $U \subseteq X-$ пучок в топологии Зариского на $X$.

ЗАМЕЧАНИЕ 4.4.10. $W^{G_{F \mid k(X)}} \cap W_{v}$ зависит только от ограничения $v$ на $k(X)$, поскольку множество $G_{F \mid k(X)}$-орбит $G_{F \mid k(X)} \backslash G / G_{v}$ нормирований $F$ совпадает, по предложению 2.4.3, с множеством дискретных нормирований $k(X)$ ранга $\leqslant r$. Например, если ограничение $v$ на $k(X)$ тривиально, то $W^{G_{F \mid k(X)}} \subseteq W_{v}$.

Примеры 4.4.11. (i) Если $V=\mathbb{Q}[\{L \stackrel{/ k}{\hookrightarrow} F\}]$ или $V=F[\{L \stackrel{/ k}{\hookrightarrow} F\}]$, то $\mathscr{V}(U)=0$ для любого нетривиального расширения полей $L \mid k$ конечного типа и любого гладкого $U$ над $k$.

(ii) Если $V=\Omega_{F \mid k}^{\bullet}$, то $\mathscr{V}(U)=\Omega_{\mathscr{O}(U) \mid k}^{\bullet}$ для любого гладкого $U$ над $k$.

(iii) Если $V=\operatorname{Sym}_{F}^{s} \Omega_{F \mid k}^{1}$, то $\mathscr{V}(U) \subset \operatorname{Sym}_{k(U)}^{s} \Omega_{k(U) \mid k}^{1}$ состоит из элементов с полюсами (по отношению к решетке $\operatorname{Sym}_{\mathscr{O}(U)}^{s} \Omega_{\mathscr{O}(U) \mid k}^{1}$ ) порядка $<s$ для любой гладкой кривой $U$ над $k$.

Заметим, что $\mathscr{V}$ функториально по отношению ко всем морфизмам гладких многообразий над $k ; \Gamma(V)$ "гомотопически инвариантно" тогда и только тогда, когда $s=1$.

(iv) Если $V=W \otimes F$ для некоторого $W \in \mathscr{I}_{G}$, то $\mathscr{V}(U)=\left(W^{G_{F \mid \overline{k(U)}}} \otimes\right.$ $\overline{\mathscr{O}(U)})^{G_{\overline{(U)} \mid k(U)}}$ для любого неприводимого гладкого аффинного $U$ над $k$, где $\overline{\mathscr{O}(U)}$ - целое замыкание алгебры $\mathscr{O}(U)$ в $F$. 
Рассмотрим следующий сайт $\mathfrak{H}$. Объекты $\mathfrak{H}$ - это гладкие многообразия над $k$. Морфизмы в $\mathfrak{H}$ - локально доминантные морфизмы, преобразующие недоминантные дивизоры в дивизоры. Покрытия - гладкие морфизмы, сюръективные над общей точкой каждого дивизора снизу. Обозначим через $\operatorname{Shv}(\mathfrak{H})$ категорию пучков на $\mathfrak{H}$. Рассмотрим функтор $\Phi: \operatorname{Shv}(\mathfrak{H}) \rightarrow \mathscr{S} m_{G}$, заданный формулой $\mathscr{F} \mapsto \mathscr{F}(F):=\lim _{A} \mathscr{F}(\operatorname{Spec}(A)) \in \mathscr{S} m_{G}$. Здесь $A$ пробегает гладкие $k$-подалгебры $F$. (Пример: если $j \leqslant 1$, то $\mathscr{F}: X \mapsto Z^{j}\left(X_{L}\right)$ - пучок на $\mathfrak{H}$ и $\mathscr{F}(F)=Z^{j}\left(L \otimes_{k} F\right)$; в частности, $\Phi$ не строг, поскольку $\mathscr{F}(F)=0$, если $j=1$ и $L=k$.)

ПРЕДЛОЖЕНИЕ 4.4.12. Въбор вложений в $F$ над $k$ полей функций всех неприводимых $k$-многообразий определяет функтор $\mathscr{S} m_{G} \rightarrow \operatorname{Shv}(\mathfrak{H}), V \mapsto \mathscr{V}$.

Вопрос: сопряжен ли он справа к $\Phi$ ?

\section{Функтор "специализации”.}

Лемма 4.4.13. Если $r=1$ u $n=\infty$, то $H_{0}\left(G_{v}^{\dagger},{ }_{v}\right)$ задает функторы $\mathscr{S} m_{G} \rightarrow \mathscr{S} m_{G_{\kappa(v) \mid k}} u \mathscr{I}_{G} \rightarrow \mathscr{I}_{G_{\kappa(v) \mid k}}$. Eстественные гомоморфизмы $G_{\kappa(v) \mid k^{-}}$ модулей $W^{G_{F \mid F^{\prime}}} \rightarrow H_{0}\left(G_{v}^{\dagger}, W_{v}\right)$ сюрвективны для всех $W \in \mathscr{S} m_{G}$. Они являются изоморфизмами, если функтор $(-)_{v}$ точен.

СлЕДСТвИЕ 4.4.14. Для любого неприводимого дивизора D на любом неприводимом многообразии $X$ над $k$ имеется естественный морфизм $C_{k(D)} \rightarrow$ $C_{k(X)}$, если $(-)_{v}$ точен при $r=1$.

Это было бы очевидно, если бы была верна первая часть гипотезы 1.1.11.

Лемма 4.4.15. Пусть $\mathscr{F}$ - некоторый функтор на категории гладких многообразий над $k$ (и всех их морфизмов). Предположим, что $\mathscr{F}\left(\mathscr{O}_{v}\right)=\mathscr{F}(F)_{v}$ (см. текст после предложения 3.4.7). Тогда $H_{0}\left(G_{v}^{\dagger}, \mathscr{F}(F)_{v}\right)=\mathscr{F}(\kappa(v))$.

Примеры 4.4.16. (i) Для гладкого полного $k$-многообразия $X$ и $q \geqslant 0$ функтор $\mathscr{F}: Y \mapsto C H^{q}\left(X \times_{k} Y\right)$ удовлетворяет предположениям леммы 4.4.15 и имеется равенство $\mathscr{F}(F)=C H^{q}\left(X_{F}\right)$.

Изоморфизм $H_{0}\left(G_{v}^{\dagger}, C H^{q}\left(X_{F}\right)\right)=C H^{q}\left(X_{\kappa(v)}\right)$ - не что иное, как гомоморфизм специализации $C H^{q}\left(X_{F}\right) \rightarrow C H^{q}\left(X_{\kappa(v)}\right)$ (см. [44]), который $G_{v}^{\dagger}$-инвариантен и потому пропускается через коинварианты $H_{0}\left(G_{v}^{\dagger}, C H^{q}\left(X_{F}\right)\right)$.

(ii) Функтор $\mathscr{F}: Y \mapsto \Gamma\left(\bar{Y}, \Omega_{\bar{Y} \mid k}^{\bullet}\right)$, где $\bar{Y}$ - гладкая компактификация $Y$, также удовлетворяет условиям леммы 4.4.15 и $\mathscr{F}(F)=\mathscr{F}(F)_{v}=\Omega_{F \mid k, \text { рег}}^{\bullet}$.

Редукция по модулю максимального идеала индуцирует сюръекцию $\Omega_{\mathscr{O}_{v} \mid k} \rightarrow$ $\Omega_{\kappa(v) \mid k}^{\bullet}$ и изоморфизм $H_{0}\left(G_{v}^{\dagger}, \Omega_{F \mid k, \text { рег }}^{\bullet}\right)=\Omega_{\kappa(v) \mid k, \text { peг }}^{\bullet}$

(iii) $\mathscr{F}: Y \mapsto \mathbb{Q}[\mathscr{O}(Y)]$ - пример функтора с $\mathscr{F}\left(\mathscr{O}_{v}\right)=\mathbb{Q}\left[\mathscr{O}_{v}\right] \neq \mathscr{F}(F)_{v}=$ $\mathbb{Q}\left[\mathscr{O}_{v} \backslash\left(k+\mathfrak{m}_{v}\right)\right] \oplus \mathbb{Q}[k]$. Однако и в этом случае $H_{0}\left(G_{v}^{\dagger}, \mathscr{F}(F)_{v}\right)=\mathscr{F}(\kappa(v))=$ $\mathbb{Q}[\kappa(v)]$.

СлЕДСТвиЕ 4.4.17. Пусть $X$ - неприводимое многообразие над $k$ с полем функций, вложенным в $F$, и $Y \subset X$ - неприводимый дивизор. Дискретные нормирования $v: F^{\times} / k^{\times} \rightarrow \Gamma$ поля $F$ ранга 1 такие, что $k(X) \cap \mathscr{O}_{v}=\mathscr{O}_{X, Y}$ 
$\left(\right.$ так что $\left.\kappa\left(\left.v\right|_{k(X)}\right)=k(Y)\right)$, образуют единую $G_{F \mid k(X)}$-орбиту. Тогда любое вложение $k(Y) \stackrel{/ k}{\hookrightarrow} F$ индуцирует канонический изоморфизм $W^{G_{F \mid k(Y)}} \stackrel{\sim}{\longrightarrow}$ $H_{0}\left(G_{v}^{\dagger}, W_{v}\right)^{G_{F \mid k(X)} \cap G_{v}}$, если $(-)_{v}$ точен.

ДокАЗАТЕльство. По лемме 4.4.13, $W^{G_{F \mid F^{\prime}}} \stackrel{\sim}{\longrightarrow} H_{0}\left(G_{v}^{\dagger}, W_{v}\right)$. Можно показать, что последовательность $1 \rightarrow G_{F \mid L} \cap G_{v}^{\dagger} \rightarrow G_{F \mid L} \cap G_{v} \rightarrow G_{\kappa(v) \mid \kappa\left(\left.v\right|_{L}\right)} \rightarrow 1$ точна, откуда следует, что

$$
\left(W^{G_{F \mid F^{\prime}}}\right)^{\left.G_{\kappa(v) \mid \kappa(v \mid}\right)} \stackrel{\sim}{\longrightarrow} H_{0}\left(G_{v}^{\dagger}, W_{v}\right)^{G_{F \mid L} \cap G_{v}}=H_{0}\left(G_{v}^{\dagger}, W_{v}\right)^{G_{\kappa(v) \mid \kappa\left(\left.v\right|_{L}\right)}} .
$$

Ограничения на объекты $\mathscr{I}_{G}$ и на факторы объектов $\mathscr{I}_{G} \otimes F$. Допустим, что $n=\infty$.

Из леммы 1.2.6 получаются условия $V_{v} \otimes_{\mathscr{O}_{v}} F=V$ и $\Gamma(V) \otimes_{k} F \rightarrow V$ для любого $W \in \mathscr{I}_{G}$ и любого полулинейного фактора $V$ объекта $W \otimes F$ ("интересные объекты категории $\mathscr{C}$ гладких полулинейных представлений группы $G$ глобально порождены"), но нужно еще проверить, по крайней мере, что эти условия не пусты на множестве неприводимых объектов.

СлеДСТвиЕ 4.4.18. Пусть $\mathscr{I}_{G}^{\prime}-$ полная подкатегория $\mathscr{I}_{G}$, для обгектов $W$ которой отображение $W^{G_{F \mid F^{\prime}}} \rightarrow H_{0}\left(G_{v}^{\dagger}, W\right)$ - изоморфизм, где $v \in \mathscr{P}_{F}^{1}-$ дискретное нормирование ранга 1. Если $n=\infty$, то $\mathscr{I}_{G}^{\prime}$ - абелева категория, замкнутая относительно перехода $\kappa$ подфакторам в $\mathscr{I}_{G}$.

Доказательство использует леммы 4.1.2 и 4.4.13. Из гипотезы 1.1.11 следует, что $\mathscr{I}_{G}^{\prime}=\mathscr{I}_{G}$.

Пусть $\mathscr{I}_{G}^{+}\left(\right.$соответственно $\left.\mathscr{C}_{-}\right)$- (максимальная) полная подкатегория $\mathscr{S} m_{G}$ $($ соответственно $\mathscr{C})$, для объектов $W$ которой выполнено равенство $W=W_{v}$ (соответственно $W=F \otimes_{\mathscr{O}_{v}} W_{v}$ ). Ясно, что они замкнуты относительно взятия факторов и содержат $\mathscr{I}_{G}\left(\right.$ соответственно $\left.\mathscr{I}_{G} \otimes F\right)$.

Лемма 4.4.19. Допустим, что $(-)_{v}$ точен. Тогда $\mathscr{I}_{G}^{+}$(соответственно $\left.\mathscr{C}_{-}\right)$- подкатегория Серра в $\mathscr{S} m_{G}($ соответственно в $\mathscr{C})$. Более того, $\mathscr{I}_{G}^{+} \neq \mathscr{I}_{G}$.

Функторы включения $\mathscr{I}_{G}^{+} \hookrightarrow \mathscr{S}_{G}$ и $\mathscr{C}_{-} \hookrightarrow \mathscr{C}$ допускают правые сопряженные $W \mapsto \Gamma(W)$ u $V \mapsto \bigcap_{v}\left(F \otimes_{\mathscr{O}_{v}} V_{v}\right)$ соответственно, но не допускают левые сопряженные.

ЗАмЕЧАНИЕ 4.4.20. В предположении, что следствие 4.4 .17 верно, следующая конструкция должна задавать вполне строгий функтор из $\mathscr{I}_{G}^{+}$в категорию (бирационально инвариантных) функторов на гладких многообразиях над $k$ со всеми, не обязательно гладкими, морфизмами, который является правым квазиобратным функтора $\Phi: \mathscr{F} \mapsto \mathscr{F}(F)$ (см. текст перед предложением 4.4.12).

Мы, как обычно, считаем, что поля функций неприводимых многообразий $Y \subset X$ над $k$ вложены в $F$. Для любого $W \in \mathscr{I}_{G}^{+}$естественный гомоморфизм $W^{G_{F \mid k(X)}} \rightarrow H_{0}\left(G_{v}^{\dagger}, W\right)$ пропускается через $W^{G_{F \mid k(X)}} \rightarrow H_{0}\left(G_{v}^{\dagger}, W_{v}\right)^{G_{v} \cap G_{F \mid k(X)}}$. Согласно следствию 4.4.17, пространство $H_{0}\left(G_{v}^{\dagger}, W_{v}\right)^{G_{v} \cap G_{F \mid k(X)}}$ канонически изоморфно $W^{G_{F \mid k(Y)}}$, если $k(X) \cap \mathscr{O}_{v}=\mathscr{O}_{X, Y}$. 
4.5. Ограничения объектов $\mathscr{I}_{G}$ на специальные подгруппы Галуа и $\mathscr{I}$-индукция. В духе Хоу-Бушнелла-Куцко и др., гладкие представления локально компактной группы можно изучать, ограничивая их на открытые компактные подгруппы.

В случае группы $G$ и $n=\infty$ если ограничиться подкатегорией $\mathscr{I}_{G}$, то заменой открытых компактных подгрупп служат открытые компактные подгруппы $\mathfrak{G}$ (см. предложение 4.6.1). Зафиксируем подполе $K$ в $F$, чисто трансцендентное над $k$, над которым $F$ алгебраично.

Пусть П - множество классов изоморфизма всех (ненулевых) гладких неприводимых представлений $\rho$ группы $U:=G_{F \mid K}$ над $\mathbb{Q}$ и $W$ - гладкое представление $G$. Тогда, как $U$-модуль, $W$ изоморфен прямой сумме всех представлений $\rho \in \Pi$ с некоторыми кратностями $m(\rho) \geqslant 0$. Пусть $\sigma \in G$ - такой элемент, что $\sigma(K) \subseteq K$. Скручивание $\rho^{\sigma}$ представления $\rho$ группы $U$ и вложение $\operatorname{Hom}_{U}(\rho, W) \hookrightarrow \operatorname{Hom}_{U}\left(\rho^{\sigma}, W\right)$ были определены в замечании 3.0.7. Там отмечалось, что если $m(\rho) \neq 0$, то и $m\left(\rho^{\sigma}\right) \neq 0$. Кроме того, $m(\rho)=m\left(\rho^{\sigma}\right)$, если $\sigma(K)=K$. Как упоминалось в замечании 3.0.7, любая пара $\rho, \rho^{\prime} \in \Pi$ сплетена.

Функтор $\mathscr{I}$-индукции $\mathscr{S} m_{U} \rightarrow \mathscr{I}_{G}$ задается (при конечномерном $\rho$ ) формулой $\rho \mapsto W_{\rho}:=\mathscr{I}\left(\mathbb{Q}[G] \otimes_{\mathbb{Q}\left[G_{F \mid L^{U}}\right]} \rho\right)$, где $F^{\mathrm{ker} \rho}$ унирационально над $L$, поле $L$ конечно порождено над $k$ и $U$-инвариантно. В общем случае функтор $\mathscr{I}$-индукции определяется по аддитивности.

ГиПотезА 4.5.1. 1) Любое неприводимое гладкое представление группь $G_{F \mid K}$, где $K$ чисто трансцендентно над $k$ и $F$ алгебраччно над $K$, входит в не более чем конечное число неприводимых облектов $\mathscr{I}_{G}$.

2) Любой неприводимый обгект $\mathscr{I}_{G}$ содержит неприводимое (гладкое) представление группы $G_{F \mid K}$, которое не входит ни в какой другой неприводимый обгект $\mathscr{I}_{G}$.

ЗАмЕчАНИЕ 4.5.2. (i) Имеется много неприводимых гладких представлений группы $G_{F \mid K}$, которые не входят ни в один из объектов категории $\mathscr{I}_{G}$. Например, таковы все нетривиальные $\rho \in \Pi$ такие, что $F^{\mathrm{ker} \rho} \supseteq K$ унирационально (например, чисто трансцендентно) над $k$.

(ii) Примерами представлений $G_{F \mid K}$, входящих в единственный неприводимый объект $\mathscr{I}_{G}$, служат тривиальное одномерное и такие нетривиальные неприводимые $\rho \in \mathscr{S} m_{G_{F \mid K}}$, входящие хотя бы в один объект $\mathscr{I}_{G}$, что $F^{\mathrm{ker} \rho}$ чисто трансцендентное расширение поля функций гладкой проективной кривой над $k$ с простым (ненулевым!) якобианом.

(iii) Выведем часть 1) этой гипотезы из остальных гипотез настоящей работы.

Пусть $\rho$ - неприводимое гладкое представление группы $G_{F \mid K}$ и $F^{\mathrm{ker} \rho}-$ конечное расширение поля $K$, т. е. чисто трансцендентное расширение поля $L$ конечного типа над $k$. Если $W$ содержит $\rho$, то $G_{F \mid L}$ имеет неподвижный вектор. Если $W$ неприводим, то имеется сюръекция $C_{L} \rightarrow W$. В предположении, что верны "все гипотезы", покажем, что $C_{L}$ имеет лишь конечное число неприводимых факторов. Факторы уровня $j$ - это факторы $\operatorname{gr}_{\mathscr{F}}^{j} C_{L}$. Если $C_{L}$ совпадает с группой Чжоу $C H_{0}\left(X_{F}\right)_{\mathbb{Q}}$ для любой гладкой проективной модели $X$ расширения $L \mid k$, а фильтрация $\mathscr{F} \bullet$ совпадает с мотивной, то $\operatorname{gr}_{\mathscr{F}}^{j} C_{L}$ определяется 
мотивом $H^{2} \operatorname{dim} X-j(X, \mathbb{Q}(\operatorname{dim} X)) \cong H^{j}(X, \mathbb{Q}(j))$. Поэтому можно считать $X$ $j$-мерным. В этом случае факторы уровня $j$ - это слагаемые полупростого представления $\operatorname{gr}_{j}^{N} C H_{0}\left(X_{F}\right)_{\mathbb{Q}}=C H_{0}\left(k(X) \otimes_{k} F\right)_{\mathbb{Q}}$ конечной длины.

4.6. Альтернативные описания $\mathscr{I}_{G}$. Категорию $\mathscr{I}_{G}$ можно описать (по крайней мере) еще тремя способами: 1) как категорию невырожденных модулей над некоторой ассоциативной алгеброй с идемпотентами; 2) как полную подкатегорию в $\left.\mathscr{S} m_{\mathfrak{G}} ; 3\right)$ как категорию гомотопически инвариантных пучков векторных пространств в доминантной топологии на $k$ (см. п. 3.4).

"Гомотопически инвариантные" представления как невырожденные модули. При $n=\infty$ действие ассоциативной алгебры $\mathbb{D}_{E}:=\lim _{U} E[G / U]$ “осциллирующих" мер на $G$ (для которых измеримы все открытые подгруппы и их сдвиги), см. замечание 3.0.6, на любом объекте $\mathscr{I}_{G}(E)$ пропускается через действие ее фактора $\mathbf{C}_{E}:=\lim _{L} C_{L} \otimes E$, поскольку морфизм $E\left[G / G_{F \mid L}\right] \otimes_{E}$ $W^{G_{F \mid L}} \rightarrow W$ представлений группы $G$ пропускается через $\mathscr{I}\left(E\left[G / G_{F \mid L}\right] \otimes_{E}\right.$ $\left.W^{G_{F \mid L}}\right)=C_{L} \otimes W^{G_{F \mid L}} \rightarrow W$.

Для любой компактной подгруппы $U$ в $G$ действие алгебры Гекке $\mathscr{H}_{E}(U):=$ $h_{U} * \mathbb{D}_{E} * h_{U}$ на $W^{U}$ для любого $W \in \mathscr{I}_{G}(E)$ пропускается через действие ее фактора $\mathscr{C}_{E}(U):=h_{U} * \mathbf{C}_{E} * h_{U}$ в $\mathbf{C}_{E}$. Например, если $F^{U}$ чисто трансцендентно над $L$, а $L-$ конечного типа над $k$, то $\mathscr{C}_{E}(U)=C_{L}^{U} \otimes E=\operatorname{End}_{\mathscr{I}_{G}(E)}\left(C_{L} \otimes E\right)$.

Пусть $\mathcal{H}_{\mathscr{I}}:=\lim _{K} \lim _{L} C_{L}^{G_{F \mid K}}-$ ассоциативная алгебра без единицы с идемпотентами. Образы $h_{K}$ мер Хаара на $G_{F \mid K}$ для чисто трансцендентных расширений $K$ подполей конечного типа над $k$ в $F$, над которыми $F$ алгебраично, являются проекторами в алгебре $\mathcal{H}_{\mathscr{I}}$.

Тогда категория $\mathscr{I}_{G}$ эквивалентна категории невырожденных модулей над $\mathcal{H}_{\mathscr{I}}$, т. е. таких $W$, что $W=\mathcal{H}_{\mathscr{I}} W$.

Алгебра $\mathcal{H}_{\mathscr{I}}$ не изоморфна алгебре Гекке (локально инвариантных мер с компактными носителями) никакой из локально компактных групп, поскольку любое, например конечномерное, подпространство в $\lim _{L} C_{L}^{G}-$ левый идеал в $\mathcal{H}_{\mathscr{I}}$, чего не бывает в алгебрах Гекке. Действительно, если в алгебре Гекке локально компактной группы есть конечномерный ненулевой левый идеал, то объединение носителей мер из него компактно и левоинвариантно, а значит, группа компактна, и, следовательно, ее гладкие представления полупросты. Из миттаг-леффлеровости системы $\left(C_{L}^{G}\right)_{L}$ и теоремы $\left.1.1 .10,5\right)$ вытекает, что $\lim _{L} C_{L}^{G} \neq 0$.

Категории $\mathscr{I}_{\mathfrak{G}}$ и $\mathscr{A} d m_{\mathfrak{G}}$. Категория $\mathscr{I}_{G}$ при $n=\infty$ допускает также описание в терминах локально компактной группы $\mathfrak{G}$ из п. 2.5. А именно, определим $\mathscr{I}_{\mathfrak{G}}$ как полную подкатегорию в $\mathscr{S} m_{\mathfrak{G}}$, объекты $W$ которой обладают свойством "гомотопической инвариантности": $W^{G_{F \mid L L_{m}}}=W^{G_{F \mid L L_{m}(S)}}$ для любого $m \geqslant 1$, любого расширения $L \mid k$ в $F$ конечного типа и любого базиса трансцендентности $S$ поля $F$ над $L L_{m}$.

ПРЕДЛОЖЕНИЕ 4.6.1. Забъвающий функтор в категорию $\mathfrak{G}$-модулей индуцирует при $n=\infty$ эквивалентности категорий $\mathscr{I}_{G} \stackrel{\sim}{\longrightarrow} \mathscr{I}_{\mathfrak{G}}$ и $\mathscr{A d d m}_{G} \stackrel{\sim}{\longrightarrow}$ $\mathscr{I}_{\mathfrak{G}} \cap \mathscr{A} d m_{\mathfrak{G}}$. 
ДокАЗАТЕЛЬСтво. Чтобы построить квазиобратный функтор $\mathscr{I}_{\mathfrak{G}} \rightarrow \mathscr{I}_{G}$, требуется определить для заданных $W \in \mathscr{I}_{\mathfrak{G}}, v \in W$ и $\sigma \in G$ выражение $\sigma v$. Найдутся подполе $L \subset F$ конечного типа над $k$ и целое число $m \geqslant 1$ такие, что стабилизатор $v$ содержит $G_{F \mid L L_{m}}$. Пусть $L L_{m}=L^{\prime} L_{m^{\prime}}$, где $L^{\prime} \subset F$ конечного типа над $k$, и $L^{\prime}$ и $L_{m^{\prime}}$ алгебраически независимы над $k$.

Пусть $N>m^{\prime}$ - такое целое число, что $L^{\prime} \sigma\left(L^{\prime}\right)$ и $L_{N}$ алгебраически независимы над $k$. Возьмем любой элемент $\sigma^{\prime} \in G_{F \mid L_{N}}$ такой, что $\left.\sigma^{\prime}\right|_{L^{\prime}}=\left.\sigma\right|_{L^{\prime}}$, и положим $\sigma v:=\sigma^{\prime} v$. Тогда $\sigma v$ не зависит от выбора $N$ и элемента $\sigma^{\prime}$, так как $v \in W^{G_{F \mid L^{\prime} L_{m}}}=W^{G_{F \mid L^{\prime} L_{N}}}$.

Проверим независимость от $L^{\prime}$. Предположим, что подгруппы $G_{F \mid L^{\prime} L_{m^{\prime}}}$ и $G_{F \mid L^{\prime \prime} L_{m^{\prime \prime}}}$ фиксируют $v \in W$. Поскольку $v \in W^{G_{F \mid L^{\prime} L^{\prime \prime} L_{m^{\prime}+m^{\prime \prime}}} \text {, достаточно разо- }}$ брать случай $L^{\prime} \subseteq L^{\prime \prime}$. Как и выше, выберем такое целое $N>m^{\prime \prime}$, что $L^{\prime \prime} \sigma\left(L^{\prime \prime}\right)$ и $L_{N}$ алгебраически независимы над $k$, и некоторый элемент $\sigma^{\prime \prime} \in G_{F \mid L_{N}}$ такой, что $\left.\sigma^{\prime \prime}\right|_{L^{\prime \prime}}=\left.\sigma\right|_{L^{\prime \prime}}$. Тогда $\sigma^{\prime \prime}$ может также служить в качестве $\sigma^{\prime}$, т. е. $\sigma^{\prime \prime} v=\sigma^{\prime} v$.

Это задает отображение $G \times W \rightarrow W$. Ясно, что это - линейное действие и что стабилизатор $v$ содержит открытую подгруппу $G_{F \mid L^{\prime}}$, и, значит, $W$ становится объектом $\mathscr{I}_{G}$.

Поскольку $L_{j}$ чисто трансцендентно над $L_{j+1}$ для любого $j \gg 1$ и допустимые представления $G$ "гомотопически инвариантны", забывающий функтор индуцирует $\mathscr{A} d m_{G} \rightarrow \mathscr{A} d m_{\mathfrak{G}}$, задавая тем самым вторую из эквивалентностей категорий.

ЗАмечАние 4.6.2. (i) Существуют допустимые, но не "гомотопически инвариантные" представления $\mathfrak{G}$. Например, $\mathbb{Q}(\rho) \notin \mathscr{I}_{\mathfrak{G}}$ для любого нетривиального характера $\rho$ группы $\mathfrak{G}$.

(ii) $\mathscr{I}_{\mathfrak{G}}$ замкнута относительно перехода к подфакторам и прямым произведениям (см. замечание 3.0 .2$, в)), но не относительно расширений в $\mathscr{S} m_{\mathfrak{G}}$. Поскольку любой морфизм из $W \in \mathscr{S} m_{\mathfrak{G}}$ в объект категории $\mathscr{I}_{\mathfrak{G}}$ пропускается через каноническое отображение в прямое произведение по всем морфизмам из $W$ в представителей всех классов изоморфизма в $\mathscr{I}_{\mathfrak{G}}$, то существует функтор $\mathscr{I}: \mathscr{S}_{\mathfrak{G}} \rightarrow \mathscr{I}_{\mathfrak{G}}$, сопряженный слева к функтору включения $\mathscr{I}_{\mathfrak{G}} \hookrightarrow \mathscr{S} m_{\mathfrak{G}}$. Функтор $\mathscr{I}$-индукции из п. 4.5 - это композиция коиндуцирования $\mathscr{S} m_{U} \rightarrow \mathscr{S} m_{\mathfrak{G}}, \mathscr{I}$ и эквивалентности из предложения 4.6.1.

Я благодарен Уве Яннсену за интерес к этой работе, многочисленные советы и обсуждения. Я признателен Институту Высших Научных Исследований (IHES) в Бюр-сюр-Иветт, Математическому институту им. Макса Планка в Бонне и Университету Регенсбурга за гостеприимство.

\section{Список литературы}

[1] N. Jacobson, Lectures in abstract algebra. Vol. III: Theory of fields and Galois theory, D. Van Nostrand Co., Inc., Princeton, NJ-Toronto, ON-London-New York, 1964.

[2] И. И. Пятецкий-Шапиро, И. Р. Шафаревич, “Теория Галуа трансцендентных расширений и униформизация", Изв. АН СССР. Сер. матем., 30 (1966), 671-704; англ. пер.: I. I. Pjatetskii-Šapiro, I. R. Šafarevič, "Galois theory of transcendental extensions and uniformization", Amer. Math. Soc. Transl. (2), 69 (1968), 111-145. 
[3] Г. Шимура, Введение в арифметическую теорию автоморфных функиий, Мир, M., 1973; пер. с англ.: G. Shimura, "Introduction to the arithmetic theory of automorphic functions", Kanô Memorial Lectures, No. 1, Publications of the Mathematical Society of Japan, 11, Iwanami Shoten, Publ., Tokyo; Princeton Univ. Press, Princeton, NJ, 1971.

[4] Я. Ихара, "О задачах конгруэнц-монодромии”, Математика (сб. переводов), 14:3 (1970), 40-98; 14:4 (1970), 48-77; 14:5 (1970), 62-101; пер. с англ.: Y. Ihara, On congruence monodromy problems, vol. 1, Lecture Notes, 1, Tokyo, 1968.

[5] W. Krull, "Galoissche Theorie der unendlichen algebraischen Erweiterungen", Math. Ann., 100:1 (1928), 687-698.

[6] U. Jannsen, "Motives, numerical equivalence, and semi-simplicity", Invent. Math., 107:3 (1992), 447-452.

[7] M. Rovinsky, "Motives and admissible representations of automorphism groups of fields", Math. Z., 249:1 (2005), 163-221; On certain representations of automorphism groups of an algebraically closed field, arXiv: math/0101170, 2001.

[8] A. A. Beilinson, "Height pairing between algebraic cycles", K-theory, arithmetic and geometry (Moscow, 1984-1986), Lecture Notes in Math., 1289, Springer, Berlin, 1987, $1-26$.

[9] S. Bloch, Lectures on algebraic cycles, Duke Univ. Math. Ser., IV, Duke University, Mathematics Department, Durham, NC, 1980.

[10] A. Beilinson, "Remarks on $n$-motives and correspondences at generic point", Motives, polylogarithms and Hodge theory, Part I (Irvine, CA, 1998), Int. Press Lect. Ser., 3, I, Int. Press, Somerville, MA, 2002, 35-46.

[11] M. Rovinsky, Generic cycles, Preprint of the Max-Planck-Institut für Mathematik, MPIM1997-80; http://www .mpim-bonn.mpg.de/preprints/send?bid=628.

[12] M. Rovinsky, "Semilinear representations of PGL", Selecta Math., 11:3-4 (2005), 491-522; arXiv: math/0306333, 2003.

[13] Д. Мамфорд, "Рациональная эквивалентность нульмерных циклов на поверхности", Математика (сб. переводов), 16:2 (1972), 3-10; пер. с англ.: D. Mumford, "Rational equivalence of 0-cycles on surfaces", J. Math. Kyoto Univ., 9 (1969), 195-204.

[14] А.А. Ройтман, "Рациональная эквивалентность нульмерных циклов", Матем. сб., 89(131):4(12) (1972), 569-585; англ. пер.: A. A. Rol̆tman, "Rational equivalence of zero-cycles", Math. USSR-Sb., 18:4 (1972), 571-588.

[15] U. Jannsen, M. Rovinsky, Smooth representations and sheaves, arXiv.org: $0707.3914,2007$.

[16] M. Rovinsky, "Admissible semi-linear representations", J. Reine Angew. Math., 604 (2007), 159-186; arXiv.org: math/0506043, 2005.

[17] W. Krull, "Endomorphismenringe in der Galoisschen Theorie", Aequationes Math., 2:2-3 (1969), 269-273.

[18] W. Krull, "Über den Galoisring", Math. Ann., 185:1 (1970), 25-37.

[19] M. Rovinsky, On maximal proper subgroups of field automorphism groups, arXiv.org: math/0601028, 2006.

[20] D. Lascar, "The group of automorphisms of the field of complex numbers leaving fixed the algebraic numbers is simple", Model theory of groups and automorphism groups (Blaubeuren, 1995), London Math. Soc. Lecture Note Ser., 244, Cambridge Univ. Press, Cambridge, 1997, 110-114.

[21] A. Robert, "Automorphism groups of transcendental field extensions", J. Algebra, 16:2 (1970), 252-270. 
[22] C. J. Ash, J. W. Rosenthal, "Intersections of algebraically closed fields", Ann. Pure Appl. Logic, 30:2 (1986), 103-119.

[23] O. Ore, "Theory of non-commutative polynomials", Ann. of Math. (2), 34:3 (1933), 480-508.

[24] W. Krull, "Über eine Verallgemeinerung des Normalkörperbegriffs", J. Reine Angew. Math., 191 (1953), 54-63.

[25] О. Зарисский, П. Самюэль, Коммутативная алгебра, т. 2, ИЛ, М., 1963; пер. с англ.: O. Zariski, P. Samuel, Commutative algebra, vol. II, The University Series in Higher Mathematics, D. Van Nostrand Co., Princeton, NJ-Toronto-London-New York, 1960.

[26] М. Атья, И. Макдональд, Введение в коммутативную алгебру, Мир, М., 1972; пер. с англ.: M.F. Atiyah, I. G. Macdonald, Introduction to commutative algebra, Addison-Wesley, Reading, MA-London-Don Mills, ON, 1969.

[27] Ф.А. Богомолов, “Абелевы подгруппы групп Галуа", Изв. АН CCCP, сер. матем., 55:1 (1991), 32-67; англ. пер.: F. А. Bogomolov, "Abelian subgroups of Galois groups", Math. USSR-Izv., 38:1 (1992), 27-67.

[28] F.A. Bogomolov, "On two conjectures in birational algebraic geometry", Algebraic geometry and analytic geometry (Tokyo, 1990), ICM-90 Satell. Conf. Proc., Springer, Tokyo, 1991, 26-52.

[29] F. Bogomolov, Yu. Tschinkel, "Commuting elements in Galois groups of function fields", Motives, polylogarithms and Hodge theory, Part I (Irvine, CA, 1998), Int. Press Lect. Ser., 3, I, Int. Press, Somerville, MA, 2002, 75-120.

[30] M. Rovinsky, "On certain isomorphisms between absolute Galois groups", Compositio Math., 136:1 (2003), 61-67; arXiv.org: math/0011176, 2000.

[31] Théorie des topos et cohomologie étale des schémas. Tome 1: Théorie des topos, Séminaire de Géométrie Algébrique du Bois-Marie 1963-1964, Lecture Notes in Math., 269, Springer-Verlag, Berlin-New York, 1972.

[32] П. Т. Джонстон, Теория топосов, Наука, М., 1986; пер. с англ.: Р. Т. Johnstone, Topos theory, London Mathematical Society Monographs, 10, Academic Press, London-New York-San Francisco, 1977.

[33] И.Н. Бернштейн, А. В. Зелевинский, "Представления группы $G L(n, F)$, где $F$ локальное неархимедово поле", УМH, 31:3 (1976), 5-70; англ. пер.: J. H. Bernstein, A. V. Zelevinskii, "Representations of the group $G L(n, F)$ where $F$ is a non-Archimedean local field", Russian Math. Surveys, 31:3 (1976), 1-68.

[34] J.S. Milne, Étale cohomology, Princeton Math. Ser., 33, Princeton Univ. Press, Princeton, NJ, 1980.

[35] U. Jannsen, "Motivic sheaves and filtrations on Chow groups", Motives (Seattle, WA, 1991), Proc. Sympos. Pure Math., 55, Part 1, Amer. Math. Soc., Providence, RI, 1994, 245-302.

[36] V. Voevodsky, "Triangulated categories of motives over a field", Cycles, transfers, and motivic homology theories, Ann. of Math. Stud., 143, Princeton Univ. Press, Princeton, NJ, 2000, 188-238.

[37] M. Levine, "Mixed motives", Math. Surveys Monogr., 57, Amer. Math. Soc., Providence, RI, 1998.

[38] M. Hanamura, "Mixed motives and algebraic cycles. I", Math. Res. Lett., 2:6 (1995), 811-821; "Mixed motives and algebraic cycles. III", Math. Res. Lett., 6:1 (1999), 61-82.

[39] U. Jannsen, Mixed motives and algebraic K-theory, Lecture Notes in Math., 1400, Springer-Verlag, Berlin, 1990.

[40] С. Маклейн, Категории для работающего математика, Физматлит, М., 2004; пер. с англ.: S. Mac Lane, Categories for the working mathematician, 2nd ed., Grad. Texts in Math., 5, Springer-Verlag, New York, 1998. 
[41] D. Abramovich, K. Karu, K. Matsuki, J. Włodarczyk, "Torification and factorization of birational maps", J. Amer. Math. Soc., 15 (2002), 531-572; arXiv.org: math/ 9904135, 1999.

[42] J. Włodarczyk, Toroidal varieties and the weak factorization theorem, arXiv.org: math/9904076, 1999.

[43] Ю. И. Манин, "Соответствия, мотивы и моноидальные преобразования", Матем. сб., 77(119):4 (1968), 475-507; англ. пер.: Ju. I. Manin, "Correspondences, motifs and monoidal transformations", Math. USSR-Sb., 6:4 (1968), 439-470.

[44] P. Samuel, "Rational equivalence of arbitrary cycles", Amer. J. Math., 78:2 (1956), 383-400.

M. 3. Ровинский (M. Z. Rovinskii)

Математический институт им. В. А. Стеклова РАН;

Независимый Московский университет

E-mail: marat@mccme.ru
Поступила в редакцию

04.08.2007 